BOND GRAPH MODELS FOR HUMAN BEHAVIOR

\author{
A Dissertation \\ Presented to \\ The Graduate Faculty of The University of Akron
}

\author{
In Partial Fulfillment \\ of the Requirements for the Degree
}

Doctor of Philosophy

Abdelrhman Mahamadi

December, 2016 


\title{
BOND GRAPH MODELS FOR HUMAN BEHAVIOR
}

\author{
Abdelrhman Mahamadi
}

Dissertation

Approved:

Advisor

Dr. Shivakumar Sastry

Committee Member

Dr. Nghi Tran

Committee Member

Dr. Jin Kocsis

Committee Member

Dr. William H. Schneider IV

Committee Member

Dr. Victor E. Pinheiro
Accepted:

Interim Department Chair

Dr. Joan E. Carletta

Interim Dean of the College

Dr. Donald P. Visco Jr.

Dean of the Graduate School

Dr. Chand Midha

Date 


\begin{abstract}
Modeling and reasoning about human behavior is an interesting problem for a variety of emerging applications across many domains. The particular interest of this dissertation is related to wellness management; specifically, the goal is to understand how to stimulate positive behaviors in individuals that lead to better management of their own wellness. The ultimate objective is to keep healthy people healthy. This research presents a Bond Graph approach for modeling human behavior as an alternative to the currently used fluid analogy models. The Bond Graph approach focuses on energy transfer and resolves potential ambiguities that could arise in the fluid analogy approach.
\end{abstract}

The dissertation demonstrates that, the Bond Graph approach also offers a comprehensive modeling framework to model, analyze and reason about human behaviors. The approach is extended to design novel controllers for wellness interventions in humans. These controllers guide the selection of wellness actions and predict the expected behavior. Further, the work also includes new schemes to diagnose human behaviors and offer analysis for unexpected behavior to support the interventions and achieve target behaviors necessary to enable large-scale societal change. 


\section{ACKNOWLEDGMENTS}

I would like to articulate my praise and gratitude to Almighty Allah for all his blessings throughout my life and during this dissertation work.

I would also like to express my sincere gratitude to my advisor, Dr. Shivakumar Sastry, who was extremely supportive, available and very encouraging.

I would also like to thank my dissertation committee, Drs. Nghi Tran, Jin

Kocsis, William H. Schneider IV and Victor Pinheiro for their valuable suggestions on my work. The support from the Department of Electrical and Computer Engineering at the University of Akron is also highly appreciated. I sincerely acknowledge and appreciate the support from National Science Foundation through award IIS-1237069 that helped to complete my graduate studies at the University of Akron.

I am so thankful to my beautiful wife who dedicated her life to me. I am also thankful to my family loving mom, encouraging dad, my brothers, my lovely sister, my son and my daughter for their non-stopping love, support, encouragement and trust during my graduate studies especially and my life generally. 
TABLE OF CONTENTS

Page

LIST OF TABLES . . . . . . . . . . . . . . . . . . . . vii

LIST OF FIGURES . . . . . . . . . . . . . . . . . . viii CHAPTER

I. INTRODUCTION . . . . . . . . . . . . . . . . 1

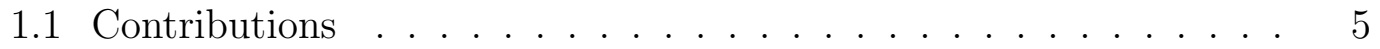

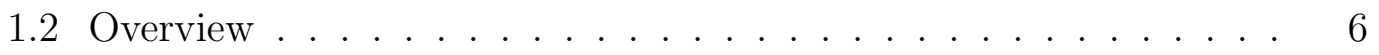

II. BACKGROUND AND RELATED WORK $\ldots \ldots \ldots \ldots$

2.1 Human Behavior Modeling Survey . . . . . . . . . . . . . 7

2.2 Bond Graph Models . . . . . . . . . . . . . . . . . . . . 16

2.3 Bond Graph Applications Survey . . . . . . . . . . . . . . . 26

III. BOND GRAPH MODELS FOR WELLNESS MANAGEMENT . . . 35

3.1 Models for Human Behavioral Change . . . . . . . . . . . . . 38

3.2 Bond Graphs Models for Human Behavior Change . . . . . . . . 45

3.3 System Analysis . . . . . . . . . . . . . . . . . . . . . . 49

3.4 Evaluation . . . . . . . . . . . . . . . . 54

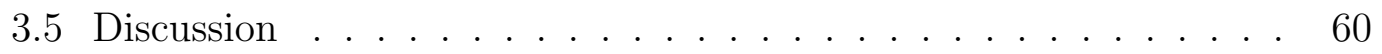

IV. BOND GRAPH MODELS FOR THE CLIENT-THERAPIST

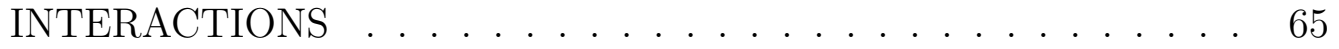


4.1 The Basic Client-Therapist Relation Model . . . . . . . . . . . . . 66

4.2 Client-Therapist Relation Using The Theory of Planned Behavior 76

4.3 Client-Therapist Relation Using The Social Cognitive Theory . . . 92

V. DIAGNOSIS OF HUMAN BEHAVIOR USING BOND GRAPH AND ANALYTICAL REDUNDANCY RELATIONS . . . . . . . . . 111

5.1 Analysis and Fault Detection . . . . . . . . . . . . . . . 112

5.2 Evaluation . . . . . . . . . . . . . . . . . 133

VI. CONCLUSIONS AND FUTURE WORK . . . . . . . . . . . 139

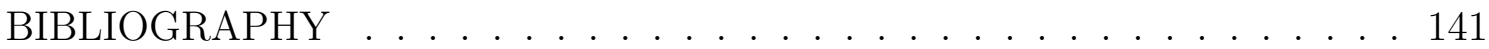




\section{LIST OF TABLES}

Table $\quad$ Page

4.1 Physical components of the system and corresponding bond graph elements . . . . . . . . . . . . . . . . . 68

5.1 Physical examples and human behavior examples for different fault types 115 


\section{LIST OF FIGURES}

Figure Page

2.1 Commonly used bond graph elements . . . . . . . . . . . . . . . 18

2.2 A simple two-tank system. . . . . . . . . . . . . . . . . . . . 24

2.3 Bond graph model for the two-tank system. . . . . . . . . . . . . . 25

3.1 Fluid analogy model based on the Theory of Planned Behavior as presented in $[1,2] . \ldots \ldots \ldots$. . . . . . . . . . . . . . 40

3.2 Fluid analogy of the simplified Social Cognitive Theory as presented in [3]. 41

3.3 Bond graph model for the two tank system assuming that $R$ is a Control Valve. . . . . . . . . . . . . . . . . . . . . . . . . . 43

3.4 Bond graph model based on the Theory of Planned Behavior. Note, the sources of flow $\zeta_{i}$ represent disturbances. . . . . . . . . . . . . . . 46

3.5 Bond graph model based on the simplified Social Cognitive Theory. . . 48

3.6 Values of the state variables in response to a step input of 1 at all the three inputs. Notice that Behavior is most closely related to Intention as can be noticed from the bond graph model in Figure 4.8. . 51

3.7 Values of the state variables when the inputs are sinusoidal, the response is different. Notice that Behavior is most closely related to Intention as can be noticed from the bond graph model in Figure 4.8. 52

3.8 The phase portraits for the psychotherapy relation . . . . . . . . . . 53

3.9 The weight dynamics for the participant in three scenarios. Scenario 1 is the baseline. The participant is resistant to change in Scenario 2 and the participant diligently complies with the recommendations in Scenario 3 as computed using the TPB bond graph model. . . . . . . 56 
3.10 When the resistance to PBC intervention is low, the participant responds promptly (top), behavior improves and the weight loss is achieved as desired. When the resistance is high (bottom), the behavior is undesirable and weight loss is not achieved. . . . . . . . 57

3.11 The weight dynamics for the participant in three scenarios. Scenario 1 is the baseline. The participant is resistant to change in Scenario 2 and the participant diligently complies with the recommendations in Scenario 3 as computed using the SCT bond graph model. . . . . . . 59

3.12 When the resistance to external cues intervention is low, the participant responds promptly (top), behavior improves and the weight loss is achieved as desired. When the resistance is high (bottom), the behavior is undesirable and weight loss is not achieved. . . . . . . . 60

3.13 Hybrid bond graph model for the Theory of Planned Behavior. . . . . . 63

4.1 Fluid analogy representation for the interaction between a Therapist

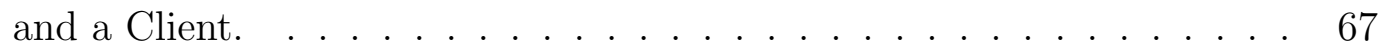

4.2 Bond graph representation for the Client-Therapist model . . . . . . . . 69

4.3 The step response of the client valence under the proportional controller 72

4.4 The step response of the client valence under the proportional and integral controller . . . . . . . . . . . . . . . . 73

4.5 The step response of the client valence under the proportional, integral and derivative controller . . . . . . . . . . . . . . 74

4.6 The phase portraits for the psychotherapy relation . . . . . . . 75

4.7 Block diagram of the Theory of Planned Behavior. . . . . . . . . . . 77

4.8 Bond graph model based on the Theory of Planned Behavior [4]. . . . . 78

4.9 Client-Therapist block diagram based on the Theory of Planned Behavior. 80

4.10 Bond graph model for the Client-Therapist relation based on the Theory of Planned Behavior. . . . . . . . . . . . . . . . . . . 81

4.11 The position of the poles on the Complex Plane indicate that the system is critically stable. . . . . . . . . . . . 85

4.12 Gain and phase Bode plots for the Behavior output of the TPB. . . . . 87 
4.13 Plot shows the best input variable to be chosen as manipulated variable in each combination of resistances values in the Theory of Planned Behavior model. Notice that, the Control belief is recommended in eight out of the nine regions . . . . . . . . . . 88

4.14 Control block diagram of the TPB model integrated with a PID controller. 90

4.15 The response of the individual's Behavioral level for a step change of 11 when Control belief $\left(\xi_{3}\right)$ is used as controlled variable. . . . . . . 91

4.16 The response of the individual's Behavioral level for a step change of 11 when Behavior belief $\left(\xi_{1}\right)$ is used as controlled variable. . . . . . . 92

4.17 Block diagram of the Social Cognitive Theory. . . . . . . . . . . . . . 93

4.18 Block diagram of the simplified Social Cognitive Theory. . . . . . . . . 94

4.19 Bond graph model based on the Social Cognitive Theory. . . . . . . . . 96

4.20 Bond graph model based on the Simplified Social Cognitive Theory. . . 97

4.21 Client-Therapist block diagram based on the Social Cognitive Theory. . 100

4.22 Client-Therapist block diagram based on the simplified Social Cognitive Theory. . . . . . . . . . . . . . . . . . . . . . 100

4.23 Bond graph for the Client-Therapist model based on the Social Cognitive Theory. . . . . . . . . . . . . . . . . . . . . . . 101

4.24 Bond graph for the Client-Therapist model based on the simplified Social Cognitive Theory. . . . . . . . . . . . . . . . . . . . . 102

4.25 Bode plots for the Client-Therapist model based on the Social Cognitive Theory. . . . . . . . . . . . . . . . . . . . 107

4.26 Bode plots for the Client-Therapist model based on the simplified Social Cognitive Theory. . . . . . . . . . . . . . . . . . . . . 108

4.27 Plot shows the best input variable to be chosen as manipulated variable in each combination of resistances values in the simplified Social Cognitive Theory model. Notice that, the Reinforcement is recommended in five out of the eight regions . . . . . . . . . . 109

5.1 Block diagram illustrates the Analytical Redundancy Relation technique for system diagnosis . . . . . . . . . . . . . 116 
5.2 FDI scheme for the human behavioral models . . . . . . . . . . . 118

5.3 Fluid analogy representation for the interaction between a Therapist and a Client. . . . . . . . . . . . . . . . . . . 120

5.4 Bond graph representation for the Therapist-Client model . . . . . . . . 121

5.5 FDI scheme for basic Client-Therapist model . . . . . . . . . . . . . . . 124

5.6 Block diagram for the Theory of Planned Behavior. . . . . . . . . . . 125

5.7 Bond graph representation for the Theory of Planned Behavior model . 126

5.8 FDI scheme for the Theory of Planned Behavior model . . . . . . . . . 129

5.9 Block diagram for the simplified Social Cognitive Theory. . . . . . . . . 130

5.10 Bond graph representation for the simplified Social Cognitive Theory model . . . . . . . . . . . . . . . . . . . . 131

5.11 FDI scheme for the Social Cognitive Theory model . . . . . . . . . . . 133

5.12 Expected and observed Client's valence when a fault happens in the therapy resistor element. . . . . . . . . . . . . . . . . 135

5.13 Expected and observed Client's valence when a fault happens in the Client's storage capacity element. . . . . . . . . . . . . . . 136

5.14 Expected and observed Client's weight dynamics when a fault happens in the therapy resistor element. . . . . . . . . . . . . . . . 137

5.15 Expected and observed Client's weight dynamics when a fault happens in the Client's storage capacity element. . . . . . . . . . . . . . . 138 


\section{CHAPTER I}

\section{INTRODUCTION}

The healthcare expenditure in the USA is expected to reach $\$ 4.8$ Trillion in the

year 2021, accounting for about twenty percent of the GDP [5], with an alarming rate of increase from about $\$ 2.6$ Trillion in 2010. Sedentary lifestyles coupled with poor diet have increased the incidence of obesity and other avoidable chronic conditions. Multiple studies have shown that increasing physical activity levels can improve cardiovascular, and the overall, health and well-being of an individual; such an improvement can decrease the amount of health care spending [6]. At the core of such a critical focus on personalized wellness management are two human behaviors - nutrition behaviors and exercise behaviors. An accurate representation and analysis of human behavior can provide key insights into how these behaviors are influenced by a variety of environmental and internal factors. The objective of this investigation was to model and reason about human behavior.

The modeling and analysis of human behavior is of interest in a broad spectrum of application domains including gaming, advertising, health and wellness management, city infrastructure planning, and management of natural resources. Through an analysis of the behaviors, one can derive key insights that highlight the factors that influence behaviors and the interactions between these factors. 
In order to be useful, the models must be precise and systematic. In addition, the modeling framework must support methods, processes and tools to analyze the static and dynamic behaviors of the modeled phenomenon and draw inferences. By observing such behaviors over time, and in response to a variety of stimuli, it is possible to design methods to regulate the behaviors to achieve larger systems objectives. In the context of this investigation, the challenge is to model human behavior and use these models to understand how, and the extent to which, nutrition and exercise behaviors of humans can be regulated.

As discussed in detail in Chapter 2, the literature for human behavior models is rich. There are many approaches that have been successfully used. The foundation for human models were developed by Psychologists; several theories such as The Theory of Planned Behavior, Social Cognitive Theory and The Transtheoretical Model of Change have been proposed to explain human behavior in a variety of contexts. While these theories explain the behavior of groups of humans, they are more useful for dealing with population-scale behaviors and not at the level of a specific individual. Further, while these theories offer a rubric for explaining behavior, they cannot capture or enable reasoning about human behaviors at the level of an individual. For this reason, several actionable models have been developed in the literature over the last decades.

Modeling human behavior is complicated and challenging because of factors that span multiple dimensions. Some of these factors are internal states of the humans - e.g., how they perceive a situation, and some factors are physical or 
physiological limitations. Some factors are environmental and social while other factors are financial. Some factors are intentional and others can be unintentional. Thus, the need to account for such varied considerations complicates the models and precludes analysis if the models are not constructed in a systematic manner based on sound principles.

To address some of the above needs and, in order to make the Psychological theories more actionable, Fluid Analogy models have been proposed in the literature recently $[1,2,3,7,8,9]$ to represent and reason about human behavior. In these models, the state variables are viewed as tanks that store fluid and the interconnections and influences between the tanks are represented by using a network of pipes in which fluid can flow. Several valves regulate the rate of flow of fluid in the pipes. Hence, in this view by using the principle of conservation of mass, one can formulate the dynamic behavior of the system by relating the levels of fluid in the tanks with the rates of flow in the pipes, over time. This framework was used to construct fluid flow analogies for the different theories of human behavior. These models were used to design controllers and successfully applied to design interventions in applications such as smoking cessation and pregnancy management.

As will be explained in Section 3.1.2, there is a source of potential ambiguity in the fluid analogy models. This ambiguity arises because two kinds of valves can be used to regulate the flow in pipes. When a conventional valve is used, the flow is regulated by the pressures in the source and destination tanks. On the other hand, when a control valve is used, energy to create the flow must be applied from an 
external source. The ambiguity arises because the fluid analogy models do not force the modeler to account for all the energy transfers in the system.

To mitigate the effects of the above ambiguity, this thesis proposes Bond Graph models for human behaviors. A Bond Graph is a domain independent model for systems and squarely focuses on energy transfer between components. It is shown that when Bond Graph models are constructed, a critical step necessary for model construction — Causality Analysis — forces the modeler to account for all the energy transfers involved in the system. In addition to reducing this ambiguity, the Bond Graph models are also relatively easy to construct.

This dissertation proposes new Bond Graph models for human behaviors. It is shown that these models are equivalent to the fluid analogy models for human behavior. The benefit of causality analysis is illustrated. The dynamic equations that are derived from the Bond Graph models eliminate the ambiguities of those derived from the fluid analogy models. It is shown that, the Bond Graph models enable analysis and reasoning about behaviors. By applying well-known methods in control theory, it is shown that the Bond Graph models are indeed a viable alternative to the fluid analogy models for human behaviors. The availability of commercial tools for Bond Graph modeling and robust algorithms for analysis makes this approach more desirable.

This dissertation also considered a well-known model for the interaction between a Client and a Therapist from the literature. By constructing a new model for this interaction, it is shown that the dynamic model reported in the literature can 
also be derived using the Bond Graph model. This interaction model was extended a more detailed model for the Client based on the well-known theories for human behavior. These extended models offer the Therapist several options for interventions. Again, using standard methods in control theory, it is shown that the approach here can help to identify the intervention options that are more likely to be effective, based on the structure of the Bond Graph models.

The third aspect of this dissertation shows that, the Bond Graph models for human behavior can be used for diagnosing and identifying the source of problems that are likely to cause a non-adherence of recommended actions. This approach is based on the well-known model-based fault detection and isolation (FDI) techniques using analytical redundancy relations. The approach was evaluated in the context of the Client-Therapist interaction model and its efficacy was proven.

\subsection{Contributions}

The specific contributions of this dissertation are:

1. First known Bond Graph models for Human Behavior.

2. Comparison of Bond Graph models and fluid analogy models for human behavior.

3. Deriving dynamic equations for Bond Graph models based on the theories of human behavior. 
4. Bond Graph models for a Client-Therapist interaction. These models provide a framework for the Client to choose a suitable Therapist. The method also offers the Therapist new "tools" to regulate the interaction.

5. Stability analysis for the client-therapist models which yields viable control options for individuals.

6. Model-based diagnosis and analysis technique for fault detection and isolation for the human behavior models

\subsection{Overview}

The remainder of this dissertation is organized as follows: Chapter 2 presents a summary of the previous work in modeling the human behavior and Bond Graph modeling. Applications of Bond Graph models are presented. Chapter 3 presents the bond graph models for human behavior and shows that bond graph approach has overcome the limitations of the fluid analogy models. The Client-Therapist interaction is presented in Chapter 4. Different models are presented along with the analysis for each model. In Chapter 5, a model-based diagnosis technique for fault detection and isolation for human behavior models is proposed. The dissertation is concluded in Chapter 6 and possible extensions and future work are presented. 


\section{CHAPTER II BACKGROUND AND RELATED WORK}

This chapter introduces the basic concepts and background of human behavior modeling and Bond Graph techniques. It is organized in two parts. The first part provides a comprehensive survey on human behavior modeling on the basis of the important domains that study human behavior modeling, their motivations and tools that were used to model human behavior in each of these domains. The remainder of this chapter provides a detailed discussion on Bond Graphs in terms of the preliminary concepts of Bond Graph modeling technique; Bond graph elements and notation followed by the procedure of creating bond graphs, assigning causalities and deriving dynamic equations. The Chapter ends with a survey on the different applications of Bond Graphs.

\subsection{Human Behavior Modeling Survey}

Human behavior models are of interest in a variety of areas that include psychology, social sciences, computer gaming, public health and in managing behaviors for programs such as smoking cessation, control of epidemics, and wellness management. Several theories and frameworks to explain human behavior at multiple scales 
have been proposed. This section outlines the major domains and the influencing researches in each domain are highlighted.

\subsubsection{Psychology}

Wide variety of approaches have been developed by psychologists over the last few decades to explain human behavior at population and individual scale. Notable among these theories are The Theory of Planned Behavior [10], Social Cognitive Theory [11], Self-Determination Theory [12], the Transtheoretical Model for Stages of Change [13] and the Health Belief Model [14].

The Theory of Planned Behavior states that attitude towards behavior, subjective norms, and the perceived behavioral control, together participate in an individual's behavioral intentions and behaviors [10]. On the other hand, the Social Cognitive Theory holds that, when a person observes any behavior and its consequences, that person memorizes the sequence of events and uses it in subsequent behaviors [11]. TheSelf-Determination Theory focuses on the motivation behind choices people make without external influence and interference [12]. The behavior change has been studied in the Transtheoretical Model for Stages of Change, which provides an assessment for the person's readiness to start a healthier behavior and proposes methods to guide the individual throughout the change stages [13]. Further, the Health Belief Model suggests that people's beliefs about health problems, perceived benefits of action and barriers to action explain engagement, or lack thereof, in health-promoting behavior [14]. 


\subsubsection{Fluid Analogy Framework}

Recent efforts have resulted in fluid analogy models for human behavior that aim to operationalize the above theories in a control systems framework $[1,2,3,7,8,9]$. These models provide an intuitive and easy approach to separate the state variables and system parameters that drive the models. Such models have been effectively used in socially relevant programs for smoking cessation and health management [9].

In $[1,2]$, the authors introduced a mathematical model to calculate the energy balance of an individual on daily basis represented as energy intake minus energy expenditure where both, intake and expenditure, were expressed in terms of Kilo Calories. Fluid analogy was used to represent the model of Theory of Planned Behavior (TPB). The outputs of TPB were sent as inputs to the energy balance model. Two different scenarios for specific male participant were considered to evaluate their model. In the first scenario, the participant immediately responds to the intervention and his attitude tank fills up rapidly, and consequently his intention and behavior tanks get filled. In the second case, the participant responds partially and slowly, which makes the valve towards the behavior and the intention partially open; hence, the result is a slow change in the behavior.

In [3], a dynamical system model of Social Cognitive Theory(SCT) was described using a fluid analogy scheme. The authors considered two different scenarios for the participant's behavior, and they observed his behavior for 20 days. First, a person with low self-efficacy was given external cue pulses on days $2,3,4,5$ and 8,9 10, 11 which resulted in few minor changes in the behavioral outcome. In the second 
case, a person with higher self-efficacy was treated using the same dose of external cues, and the response in behavioral outcome was much better. The authors validate their approach by comparing their model results to previous research results. They used the data from mobile interventions for lifestyle exercise and eating at Stanford (MILES), as baseline for comparison. Authors of [7] have presented a semi-physical system identification approach for interventions associated with improving physical activity. Their purpose was to propose an input signal design together with an estimation procedure that provides desired behavioral outcomes. The proposed strategy considers the construction of two open-loop experiments with different inputs. The first is designed based on a priori knowledge from social cognitive theory (SCT) and previous experience with behavioral interventions. This experiment provides insights regarding the dynamics of the system and leads to an initial model. Based on results from informative experiment, a second optimized experiment is designed to shape the behavioral outcomes in a desired pattern. Authors have shown that the design of the required inputs within practical operating limitations can be formulated as a feasible constrained optimization problem.

Fluid analogy approach was also used by [8], in which authors have presented dynamical system models that describe how a behavioral intervention can influence weight gain during pregnancy. Their model integrates a mechanistic energy balance with the dynamical behavioral model; TPB was used to obtain the dynamical behavioral model. It was assumed that out of the interventions, the state of the PCB (perceived control behavior) changes, and accordingly the energy intake reduces. A 
hypothetical case study was demonstrated to illustrate efficacy of their model.

Another application for the fluid analogy of human behavior is for smoking

cessation [9] . The objective was to determine the effects of motivational interviewing in promoting smoking cessation. Authors targeted the Cochrane Tobacco Addiction Group, and they looked for Randomized controlled trials in which motivational interviewing or its variants were offered to smokers to assist smoking cessation. They concluded that, motivational interviewing may assist smokers to quit. However, the results should be interpreted with caution due to variations in study quality, treatment fidelity and the possibility of publication or selective reporting bias.

\subsubsection{Control Theory}

Having dynamic models representing human behavior allows control theory scientists to propose ideas of how to maximize the healthy behavior and minimize the unwanted behavior using the concepts of control theory. In [15], authors presented a data-centric modeling and predictive control approach for behavioral intervention. A Model-on-Demand (MoD) algorithm was proposed to estimate the hybrid system at each time step and the output of the algorithm was used in the model predictive controller(MPC) of the hybrid system. Authors have considered the problem of controlling the dosage of intervention components to improve parental function and reduce disorder in at-risk children. To examine their model, they considered data from "Fast Track Program", which was multi-year program designed to prevent disorders in at-risk children. Initially the decision about which dose of counseling 
to offer each family is based primarily on the family functioning questionnaire completed by the parents. After that, their model will analyze the response of the family and decide about the next dose. It was concluded that, the proposed MoDPC is useful for the control of nonlinear hybrid systems, displaying acceptable performance levels while simplifying the task of modeling.

Another use of control theory in human behavior was proposed in [16]. The authors represented the psychotherapy relationship between the therapist and the client using mathematical control model. A model was provided to describe the interaction between therapist and client similar to an earlier work that described the Husband-Wife relationship, and control theory was applied to obtain the phase plots of the client-therapist model. They were able to show that the psychotherapy relationship has two critical points, one a stable point and the other a saddle point; they also concluded that a well-trained therapist can lead the client to the stable point.

\subsubsection{Social Science}

In this domain, scientists have focused on the population level and have studied the relation and influence on the individual. Author of [17] has claimed that, there is a number of different philosophical positions that provide an interpretation of empirical data into models. One of these positions is that, the universe and everything within the universe are represented as physical components or entities. The author proposed an approach, in which it is essential to understand the relationships be- 
tween the entities. The basis of his framework is that, the human being consists of three main aspects - mind, body and spirit. The aspect of mind is composed of: cognitive, emotional and the co-native components. The output of the individual is the overt action of organism or the behavioral system. This output is affected by: (1) The transactions among the various components of mind, (2) Biological maturation, bodily functioning, and the spiritual dimension of the individual, (3) Environment or context of the individual and (4) The reflection on feedback from the environment as a result of an individual's overt behavior. Thus humans do not develop in isolation; they develop in a variety of contexts. First level starts from the micro-system inside the family while the second level is the meso-system, which is the intermediate level of influences and includes the society. Finally the macro-system is comprised of the the influences of the global region.

Another work on social science was reported in [18], where the authors built on an existing ecological theories of health behavior, to change the effect of population-level factors in individual health behaviors. A pragmatic characterization of structural interventions was used. They examined social and environmental factors beyond individual control and mechanisms as to how they influence behavior. They were able to identify four categories of structural factors: (1) Availability of protective or harmful consumer products, (2) Physical structures (or physical characteristics of products), (3) Social structures and policies and (4) Media and cultural messages. It was concluded that interventions that target the four identified structural factors are means to prevent the adoption of high-risk behaviors. 


\subsubsection{Machine Learning}

The uncertainty in human behavior has been studied extensively; Probabilistic analysis, Markov decision process, entropy rate and statistics were used by machine learning scientists to embed the uncertainty in modeling human behavior. In [19], authors viewed human behavior as a structured sequence of context sensitive decisions. Probabilistic approach and Markov decision process were used to predict human behavior, the key challenge in their work is the uncertainty involved with the human behavior. They have employed the principle of Maximum entropy to resolve the ambiguities in choosing distributions and have successfully implemented their approach for predicting driving behavior as well as for route recommendation. Authors of [20] have described human behaviors as set of dynamic models sequenced together by Markov chain like Kalman filters. They considered the human as a device with large mental states, each has its own transition probability. To accomplish their objective, they have adopted the expectation-maximization methods with hidden Markov Models which were used to recognize human behaviors from sensory data. According to the recognized behavior they were able to predict the human next move. The model was tested using prediction of driving behavior and they have successfully achieved 95\% accuracy.

Another probabilistic analysis approach to describe human behavior is [21], in which, a molar model of age differences in spatial memory was proposed. It was assumed that the information processing occurs in a neural network, and the information can be predicted using "computational temperature" of the neural network. 
Statistical approach was used to estimate the computational temperature for both young and old adults. The goal was to determine what happens to an informationprocessing system when entropy is high in one group (older adults) compared with another (younger adults). Authors were able to validate through experiments that age differences in entropy, accounts substantially for age differences in spatial memory. Another use of Markov decision process in human behavior is discussed in [22]. In this work, Markov Decision Process (MDP) was used to build a decision support system to help humans in their wellness management. The proposed goal-seeking framework systematically integrates knowledge from multiple disciplines and offers wellness prescriptions to participants.

\subsubsection{Virtual Reality and Gaming Theory}

The importance of understanding human behavior also appears in the domain of virtual reality and gaming theory, since human behavior and attitude have to be modeled in order to simulate a human. In [23], authors proposed a design for virtual agents that simulate humans in decision making under uncertainty. To accomplish their objective, they had to quantitatively represent the human behavior. According to their results, an average human decision maker can be described by only two components - inference and selection model. In their model they assume that, there are $N$ random variables in the environment, where each random variable has a fixed distribution, that is unknown to the decision maker. At the first trial, the decision maker is asked to choose among $M$ options, then he observes independent 
samples of the random variables in that trial. The decision maker's utility in a trial depends both on choice and on the realized samples of all random variables in that trial. The objective for the decision maker is to maximize his/her utility in the whole decision period. 800 participants were recruited to get data for testing their model. Study concluded that to resemble an average person, the agent should be designed with a few human irrationalities. They have also observed that people seem to be more heterogeneous in a less uncertain environment while more homogeneous in a more uncertain environment.

Authors of $[24,25]$ have focused on challenges to improve the realism of socially intelligent agents. A particular attention was given to the impact of personality/cultural values and the need to integrate valid human performance moderator functions (PMFs) from traditionally separated subfields of the behavioral literature. A unifying architecture and principles were designed for integrating existing PMF theories and models. To validate their work, they considered several usage case studies (asymmetric warfare, civil unrest, and political leaders). They concluded that, implementing and inter-operating this broad collection of PMFs helps to open

the agenda for research on syntheses that can help the field reach a greater level of maturity. In [25], they presented the software implementation of [24].

\subsection{Bond Graph Models}

In this section, the notation of Bond Graphs is presented and the procedures of Bond Graph construction, causality assignment and dynamic equations deriving are 
explained. Further, the efficacy of using Bond Graph to modeling human behavior is demonstrated through an example.

\subsubsection{Notation and Construction}

Figure 2.1 presents the notation that is commonly used to represent the nodes and edges of a Bond Graph. For each element, the figure illustrates if the element is consuming or generating energy. The direction of the half-arrow indicates that the element consumes energy when the arrow points to the element. Otherwise, it generates energy in the system. For energy transfer to occur in any system, there must be a bidirectional transfer of effort and flow, simultaneously. This bidirectional energy transfer is represented as Bond in the model. Thus, when one element in a bond is the source of effort, the other element must be a source of flow, and vice versa. For example, the element $S_{e}$ is a source of effort; the element that is connected to $S_{e}$ must be a source of flow. The direction of flow is represented in a Bond Graph using strokes (vertical "|"). Notice that for $S_{e}$, the stroke indicates that the flow is into the source of effort. Similarly, for $S_{f}$, the stroke indicates that the flow is away from the element. The resistor element, $R$, is indifferent to the direction of flow. The relationship between effort and flow for the capacitor element, $C$, and the inductor element, $I$, can be expressed either in an integral form or a differential form. The integral forms of these elements are preferred because it forces the modeler to specify the initial conditions for these element. For this reason, the figure shows $C$ as a source of effort and $I$ as a source of flow. 


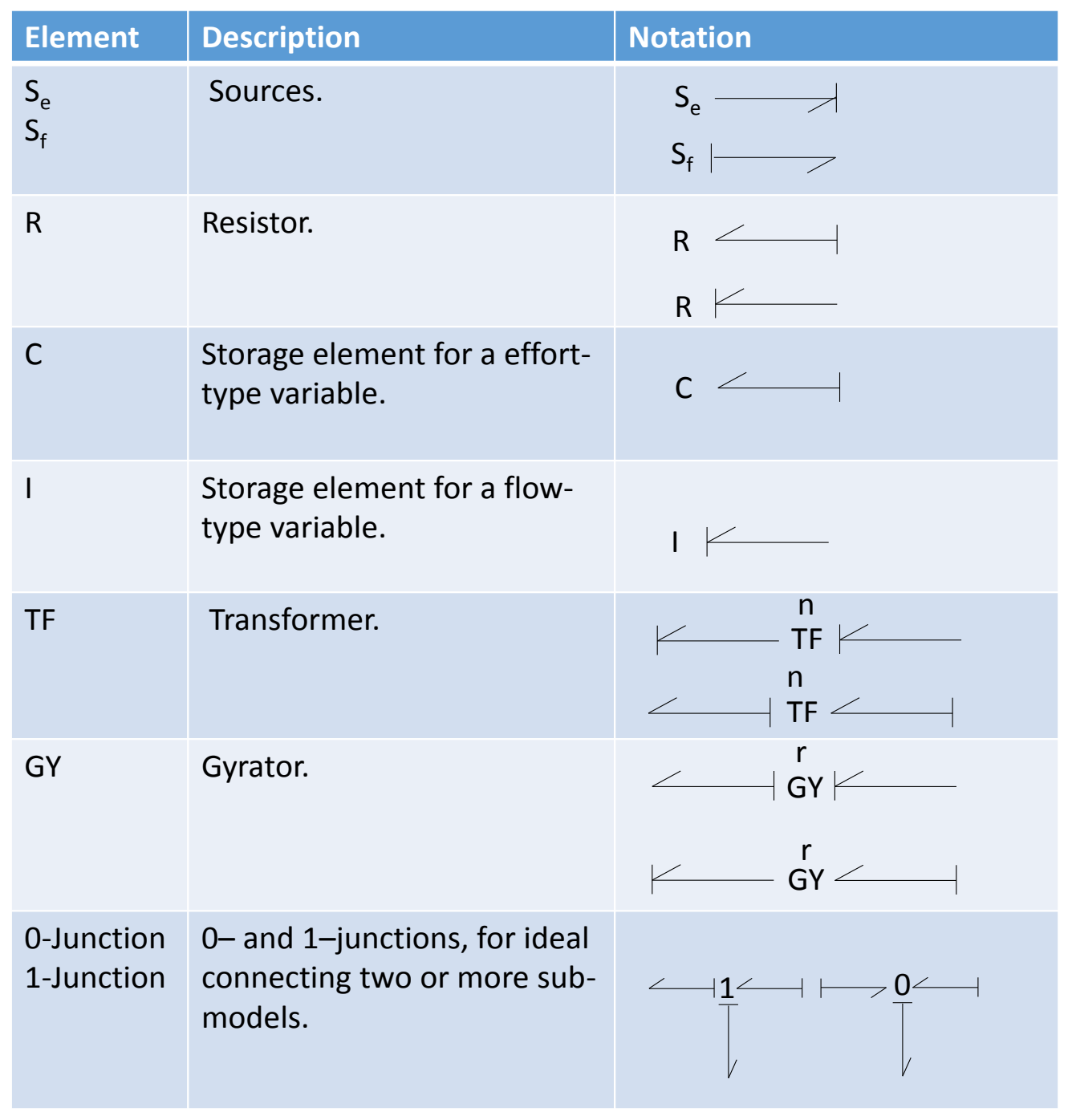

Figure 2.1: Commonly used bond graph elements 
The notion of power is important in energy transfer. Power is defined as the product of effort and flow. In many applications, it is necessary to increase the effort or the flow; however, the energy in the system cannot be increased or decreased. To achieve these transformations in the magnitude of effort or flow, a Transformer element, $T F$ can be used. When a $T F$ transforms effort, the two elements it is connecting must be sources of effort. Similarly, when a TF transforms flow, the two elements must be sources of flow. It is also necessary to transform energy from one type, i.e., from effort to flow and vice versa. This is accomplished using the Gyrator element, $G Y$. The $G Y$ element is also useful to transform energy across domains, i.e., electrical, chemical, mechanical, etc. In this case, if a $G Y$ element accumulates effort from one domain, it must generate flow in the other domain, and vice versa. These two elements, $T F$ and $G Y$, are not a source of effort or flow.

The $0-$ Junction and 1 -Junction elements are used to represent collections of elements that share effort or flow, respectively. For a 0 -Junction, exactly one element in the junction must be selected as a source of effort, i.e., only one stroke inside the junction. Similarly, for a 1-Junction, exactly one element in the junction must be selected as a source of flow, i.e., only one stroke outside the junction. In a 0-Junction, the sum of efforts must be zero (e.g., Kirchoff's Voltage Law in a series connected electric circuit), and in a 1 -Junction, the sum of flows must be zero (e.g., Kirchoff's Current Law in a parallel connected electric circuit).

The topology of the physical system guides the construction of a Bond Graph. The physical elements of a system must be identified as being one of the types of 
elements discussed above. Every distinct effort in the system must be represented by a unique 0 -Junction. All the Bond Graph elements must be connected using 1-Junctions between two corresponding 0-Junctions. Half arrows must be added to all bonds indicating the direction of flow. One source of effort must be selected as the reference and the corresponding 0 -Junction and all incident bonds must be eliminated. When TF and GY elements connect different subsystems, reference points must be selected for each subsystem. Finally, the Bond Graph must be simplified.

\subsubsection{Causality Assignment}

The objective of causality analysis is to determine whether the flow of energy in the system is consistent with the law of energy conservation [26]. The bond graph methodology admits four types of causality and by following these rules, one must assign causality to the bonds. Causality is denoted by a stroke ("|") on one end of the bond. The flow of energy in a bond is from the component on the bond without the stroke to the element that has the stroke. Note that half arrows only denote whether the corresponding physical component is generating or consuming energy. The details of assigning half arrows and causal strokes will be illustrated with a specific example after describing the four types of causality that must be considered.

1. Fixed Causality: The energy flow is constrained in one specific direction. For example, when the element at one end of a bond is a Source of Effort, the causal stroke must be at the other end, i.e., the flow is assumed to be out of the source of effort and into the element at the other end of the bond. 
2. Constrained Causality: In bond graph elements such the $T F, G Y, 0$-Junction and 1-Junction, there is a physical relation between the different ports of the element because the causality of one port imposes the causality on the other ports.

3. Preferred Causality: The storage elements $C$ and $I$ could be represented in either of integration or differentiation forms. The integration form is preferred because it forces the designer to specify an initial condition for the elements.

4. Indifferent Causality: In this case, the element does not impose any constraints on the causality. For example, in the case of a Resistor element, the flow can either be into the element or away from the element.

When carrying out the causality analysis, it is necessary to first start with the components that have fixed causalities. Usually, these are the sources. Next, it is necessary to check if any of the assigned causalities affect the elements with constrained causality, i.e., $T F, G Y$ or 0 -Junction and 1-Junction. If such connections exist, the assigned causalities must be propagated through these elements. The next step is to consider the components with preferred causality. If the preferred causalities can be assigned without violating any of the assigned causalities; any changed causalities must be propagated through the Junctions. Finally, it is necessary to check if there are unassigned causalities and the strokes are assigned to the indifferent causality elements. 
Causality analysis can serve as a diagnosis tool that can check whether or not the systems are modeled properly. When the causalities assigned are inconsistent with the rules, it usually denotes that a model change may be warranted.

\subsubsection{Dynamic Equations}

The dynamic equations of an energy system can be derived from the bond graph of that system. These equations are written in terms of states of the system. Generally, there are two kinds of states $P$ and $Q$ for each bond that supplies energy to a storage element; the $P$ state represents the integration of the effort variable and is usually used with the $I$ type storage elements; the $Q$ state is the integration of the flow variable and it is used with the $C$ type storage elements.

The concept of a strong bond is important and is related to the junction elements. Only one bond in a $1-$ junction must supply the flow, i.e., only one bond should have the causality stroke outwards. Similarly, only one bond in a $0-$ junction must supply the effort and that bond should have the causality stroke inwards. These unique causality bonds at the junctions are called strong bonds.

The procedure of deriving the dynamic equation from the bond graph is described below.

1. Start with the Sources, Inductors, Capacitors and Resistor elements and write their equations according to the causalities.

2. Write the equations of the two ports elements, i.e., $T F$ and $G Y$, and the Junction elements for the strong bonds variables. 
3. Resolve the unknown values using the values across the strong bonds.

4. Resolve the variables in the right hand side by using the states variables and system parameters.

5. Check to see if all the equations are completely reduced. If not, it means there is some loop in the system and this can be resolved by solving these equations as a system of linear equations.

\subsubsection{Illustrative Example}

Consider a simple two-tank model that is illustrated in Figure 2.2. There are two

sources of flow $S_{1}$ and $S_{2}$, and two storage tanks $T_{1}$ and $T_{2}$ that are modeled as capacitor elements. The valves $N_{1}$ and $N_{2}$ control the flow into the system from the respective sources. The valve $R$ controls the flow between the two tanks. 


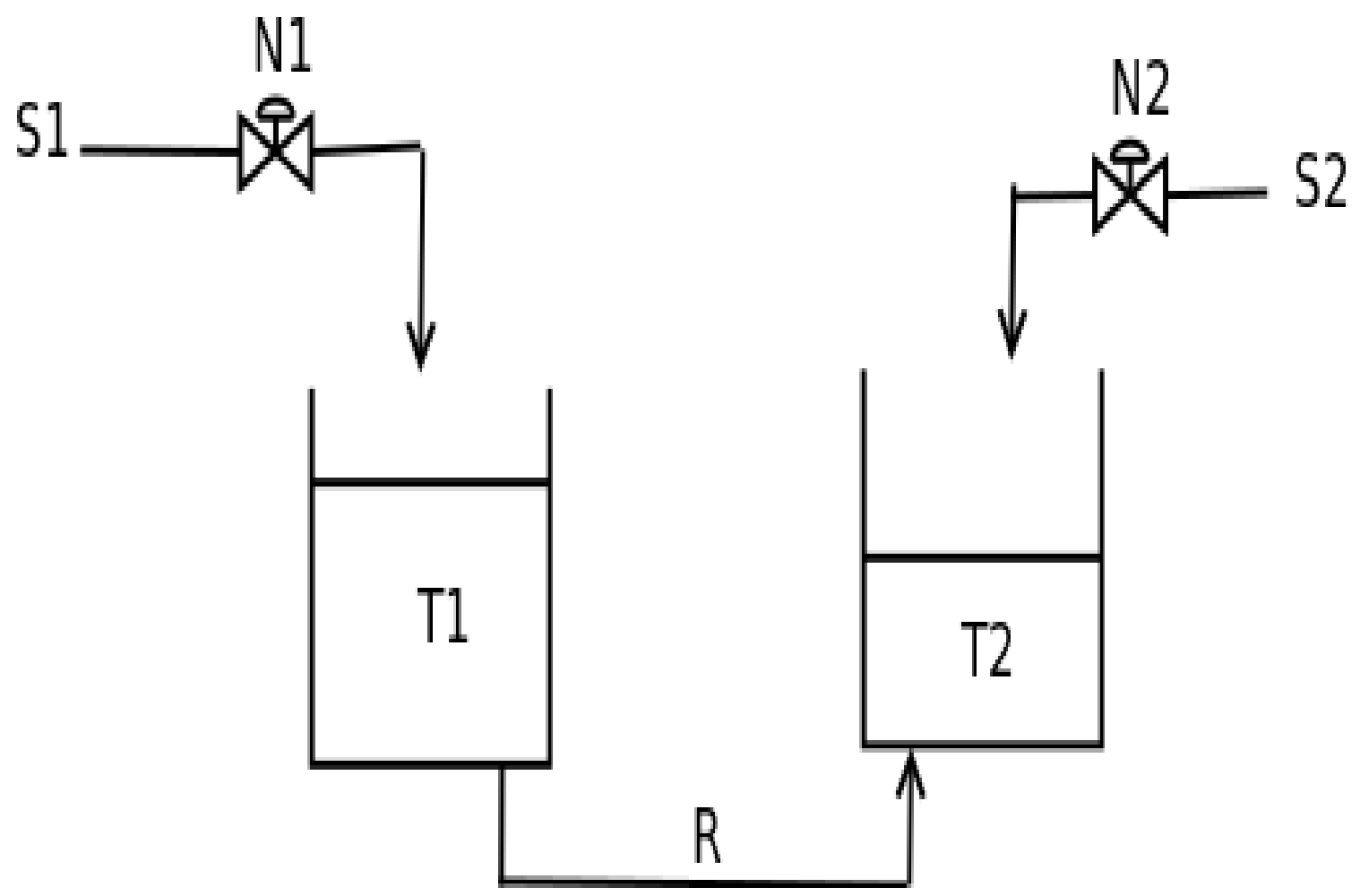

Figure 2.2: A simple two-tank system.

A Bond Graph model for the two-tank system in Figure 2.2 is shown in Figure 2.3. In this model, the two sources of flow $S_{1}$ and $S_{2}$ represent inflows into the system as shown in Figure 2.2. The tanks $T_{1}$ and $T_{2}$ are represented as storage elements $h_{1}$ and $h_{2}$ of type $C$. The valves $N_{1}$ and $N_{2}$ are modeled as $T F$ elements because they change the magnitude of the flow between the respective sources and the tanks. The valve that connects the two tanks is modeled as a resistor element $R$. The two $1-$ Junction elements represent the corresponding physical point that is between the valve and the tank in Figure 2.2 because the flow at these points is conserved. Similarly, since $R$ is a conventional valve, the pressure difference across 
the two tanks $T_{1}$ and $T_{2}$ regulates the flow through $R$, i.e., the effort is conserved. For this reason, the model in Figure 2.3 has a 0 -Junction element. The strokes on the bonds indicate the direction of flow; fluid from the sources flows into the storage elements (i.e., tanks). The flow through $R$ can either be from $T_{1}$ to $T_{2}$ or from $T_{2}$ to $T_{1}$ as the strokes indicate.

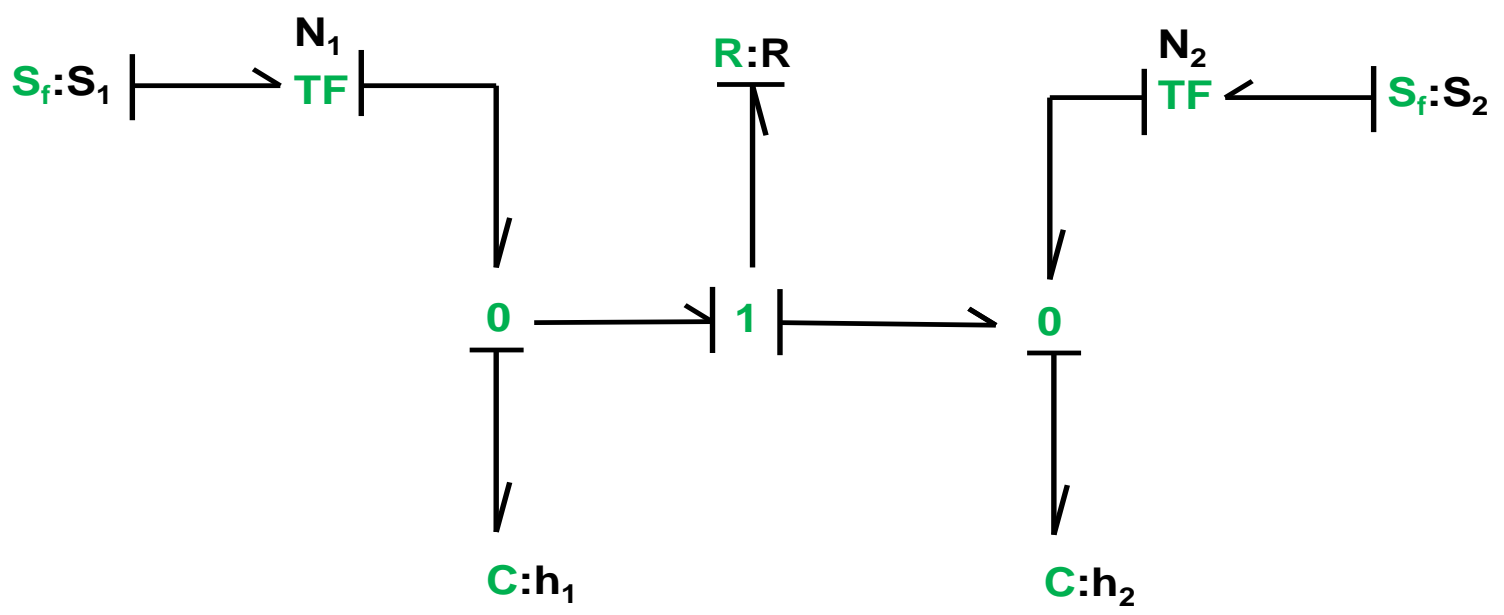

Figure 2.3: Bond graph model for the two-tank system.

The two state variables in the system are the levels of the tanks, i.e., $h_{1}$ and $h_{2}$. The dynamic equations specify how these variables change in the system. The equations derived from the bond graph model in Figure 2.2 are. 


$$
\begin{aligned}
\frac{d h_{1}}{d t} & =\frac{S_{1} N_{1}}{\rho g C_{1}}-\frac{1}{R}\left(h_{1}(t)-h_{2}(t)\right) \\
\frac{d h_{2}}{d t} & =\frac{S_{2} N_{2}}{\rho g C_{2}}+\frac{1}{R}\left(h_{1}(t)-h_{2}(t)\right)
\end{aligned}
$$

Note that these equations utilize all the components in the corresponding junctions shown in Figure 2.3.

\subsection{Bond Graph Applications Survey}

A Bond Graph is a domain independent, graphical, model for representing and reasoning about the dynamic behavior of a physical system [26]. Bond Graphs were introduced as a unifying notation for systems involving mechanical, electrical and hydraulic components [27]. Energy transfers between the components are explicitly represented in the Bond Graph approach. The components are the vertex of the Bond Graph; each node has pre-specified ports[28]. The edges (a.k.a. Bonds) of the graph represent bilateral energy transfers between nodes. Specifically, these bonds represent the bilateral signal flow of the power conjugate variables effort and flow [29] between the nodes via specific ports. Physical effects on the components and their interactions are initially considered qualitatively and specified more precisely as the modeling process evolves [30]. Irrespective of the domain, i.e., electrical, mechanical

or chemical, analogous systems have a common Bond Graph representation that can be analyzed in a common framework [31]. 
In this section, the major areas of Bond Graphs applications are introduced, and some representative results achieved in each area are presented.

\subsubsection{Electrical and Magnetic Systems}

One of the main areas that involve energy exchange is the electrical systems, such as electromagnetic circuits, both D.C. and A.C. circuits. For such systems a powerful technique is needed to model the components of the system and derive the dynamic equations. Clearly the Bond Graph approach is suitable for these applications.

For example, in [32] it was shown that it is possible to describe electromagnetic (E-M) fields with generalized Bond Graphs, i.e., the relations of E-M fields in moving matter, forces due to E-M fields and field transformations with respect to each other can be clearly represented by the Bond Graph. However, it is not possible to describe E-M phenomena in moving matter with conventional Bond Graphs, and a generalized bond graph concept is required.

Another work was done in [33], in which, a generalized Bond Graph model for rotary and linear electromechanical actuators was developed. It was also demonstrated that a wide variety of actuators can be modeled by versions of the basic Bond Graph which is simplified when some effects are negligible.

In [34], Bond Graph models were used to demonstrate that magnetic and electrostatic transducers are mathematically analogous although electrically dual. An all-mechanical equivalent model shows the non-linear behavior clearly and aids in the intuitive understanding of the problems associated with the use of such actuators. 


\subsubsection{Heat Transfer and Thermodynamic Systems}

The graphical representation of Bond Graphs such as the building blocks are useful in modeling the heat transfer and thermodynamics applications, few examples are demonstrated here.

Authors of [35] have proposed the use of Bond Graph building block approach to create models for representing the dynamics pf the heat exchanger.

In [36], authors proposed a clear graphical representation of heat exchangers using a particular type of pseudo-bond graph, which exhibits the structure of an assumed model which remains valid even for reversing flows and nonlinear element relationships.

Bond Graphs are also useful in constructing simulations, for example in [37]. The authors used a Bond Graph simulation of heat transfer through a concrete wall based upon modal analysis to be compared with that based on a finite-difference technique.

\subsubsection{Chemical Systems}

Another area of application for Bond Graph is the chemical systems in which the structure of the molecules and the reaction stages can be modeled and represented graphically. In this section, various chemical applications of Bond Graph are presented.

Molecular structure was studied in [38]. The authors proposed a characterization of molecular model surfaces. Bond Graphs were used to obtain the molecular 
skeleton which was compared to the van der Waals graph in describing the body of the molecule.

In [39], the author represented network theory for chemical reactions, and used Bond Graphs to derive the canonical set of differential equations for the reaction system.

Another chemical application of Bond Graph is in [40]. The author developed a complete system for oxygen generation from pressure swing adsorption. With the aid of Bond Graph concepts he was able to obtain the model for this system.

In [41], Bond Graphs were used to extend the chemical kinetics models to electrochemical systems. Furthermore, these Bond Graphs models were extended to include the thermal models due to energy dissipation of chemical nature.

\subsubsection{Bioengineering Systems}

Bioengineering systems involve energy conversion and energy exchange between parts as well as dynamic behavior. For this reason, Bond Graph techniques are widely used to model systems in this area.

For example, in [42], authors used Bond Graphs to develop a mathematical model in order to investigate the dynamic behavior of above-knee prosthesis in the swing phase.

Another bioengineering application is in [43], where the authors used the Bond Graphs technique to represent a network thermodynamic model for cell membrane coupled flow of water and permeable cryoprotectant additives. 


\subsubsection{Machines and Mechanical Systems}

One of the fundamental areas that uses Bond Graph approach for modeling systems and constructing simulations is the machines and mechanical systems. Because such systems involve energy conversion between domains and Bond Graph is domain independent technique.

In [44], a simple Bond Graph model of automotive power trains was proposed. Then various extensions of the basic model were also considered, including four wheel drive vehicles and power trains with automatic transmissions. The derived models were useful for preliminary assessment of the effects of power train / component design changes, and for power train control system design.

Authors of [45] have proposed the usage of the Bond Graph framework for performing the type synthesis of mechanisms.

The work in [46] demonstrates that the Bond Graph method provides a handy tool to build a model consisting of many mutually connected components in order to analyze the handling response of steering variations using different sets of tires.

\subsubsection{Hydraulics and Fluid Mechanic Systems}

Another important area for Bond Graph applications is hydraulics and fluid mechanics, here are few examples. In [47], Bond Graphs are proposed as an alternative to circuit models that provide an enhanced physical insight and computational ease. They showed the scope of Bond Graph applications using modeling transducers. 
A new approach to reduce the engine excited frame vibration in an automobile using semi-active hydraulic mount is discussed in [48]. Bond Graph approach was used in modeling their new technique.

In [49], a feasibility study of a dual-pump hydraulic supply system was presented. The pump consisted of a low-volume fixed-displacement pump and a highvolume variable-displacement pump. Bond Graph approach was used in the analysis of such systems.

\subsubsection{Economic and Social Systems}

Bond Graphs are not only useful for pure engineering applications, but also they can be used in modeling some of the economic applications. For instance, reinvestment, inventory affects, dynamic economics and constitutive relations can all be modeled and represented using Bond Graphs.

In [50], a survey was conducted of the economics and related literatures in order to recast the conventional theories into the form of constitutive relations. The authors were able to improve the modeling of engineering-economic dynamics by the bonding of the marketplace to economic populations, using bond graph concepts and bond graph representation.

In [51], the Bond Graph use was extended from describing physical systems only to the analysis of non-linear and dynamic economic systems using a specific set of rules for the construction of economic bond graphs and for the step by step derivation of the state equations that describe the dynamics of these economic systems. 
Also in [52], the possibility of describing reinvestment and inventory effects in Bond Graph terms is demonstrated. Since the associated differential equations in price and order-flow variables are bilinear forms, these effects are the essential inertia and compliance of economic bond graph theory.

\subsubsection{Programming Systems}

Bond Graph notations are useful for programming applications. In [53], authors proposed a programming system which can be used to construct models of physical systems. Bond Graph notations are used for representing the components and connections.

\subsubsection{Fault Detection and Isolation}

Bond graph Analytical Redundancy Relations (ARR) for systems diagnosis and fault analysis have been studied extensively in many domains [54, 55]. An approach for fault diagnosis of a nonlinear system using ARRs was proposed in [56]. The authors showed that ARRs are insensitive both to the state variables and the unknown parameters. The approach was validated using an example SISO model of an underwater vehicle.

A bond graph approach for fault detection and isolation (FDI) was proposed in [57]. Under the assumption that no two faults appear at the same time, they illustrated the use of residual bond graph sinks for the numerical evaluation of ARRs. The approach was validated using an example hydraulic system with two tanks. 
A bond graph approach was also proposed for modeling a traveling ultrasonic wave motor in [58]. The model was used as a reference to apply the ARR method. The model was validated by comparing the simulation behavior of an ultrasonic linear motor with experimental results from a prototype.

A hybrid bond graph (HBG) approach for modeling and diagnosing a switching system was proposed in [59]. The HBG was shown to be useful both for qualitative and for quantitative diagnosis. Global Analytical Redundancy Relationships (GARRs) were derived from the HBG model using a specific causality assignment procedure to support the quantitative diagnosis. A Temporal Causal Graph (TCG) and a directed graph were constructed to capture functional redundancy to support the qualitative diagnosis.

A qualitative parameter estimation approach for FDI based on bond graph for an automobile combustion engine cooling system was presented in [60]. A graph was used to describe the dynamic performance of this system. A small leak was created in the cooling system to verify the accuracy of the FDI.

Distributed FDI schemes have been proposed in [61]. The authors presented two different algorithms for developing and analyzing an online, distributed, modelbased diagnosis scheme for isolating sudden faults in large continuous systems. They demonstrated the effectiveness of their algorithms by applying them to the Advanced Water Recovery System developed at the NASA Johnson Space Center.

A formal modeling scheme for continuous systems that focused on diagnosis was presented in [62]. The authors offered an approach based on the bond graphs. 
They introduced schemes for the diagnosis task by converting equations to conflict sets. This representation facilitated candidate generation and the measurement selection processes.

A quantitative model-based FDI approach for hybrid systems was presented in [63]. The approach used ARRs that were obtained from a hybrid bond graph. The technique also used the standard sequential causality assignment procedure. The approach was validated using a power electronic switching circuit.

Another model-based FDI approach was proposed in [64]. Here the FDI is based on verifying the consistency of the ARRs using an interval pool. This work accounted for the uncertainty in the parameters and measurements. Information was gathered using partial derivatives of the ARRs and the candidates were eliminated according to the signs of the residuals. The technique was evaluated using a system comprising two tanks. 


\section{CHAPTER III}

\section{BOND GRAPH MODELS FOR WELLNESS MANAGEMENT}

The ability to model and reason about human behavior is critical for several applications that demand tight integration between emerging technologies and humans. The interest of this investigation is to understand the dynamics of human behavior in the context of empowering wellness management. The objective was to understand the enablers for behaviors that can improve the nutrition and physical activity levels of individuals; with this knowledge, next-generation systems designers can offer targeted and timely interventions that can keep healthy people healthy for longer periods of time.

Personalized Wellness Management (PWM) is a critical national priority that can significantly reduce healthcare costs that are currently greater than $16 \%$ of the nation's GDP [5]. Approximately $75 \%$ of the total healthcare costs are associated with management of chronic illnesses such as Diabetes and Hypertension [6]. In addition, there are high costs to our societies such as the loss of productivity and morale, and the worsening Quality of Life. To address these growing challenges, the current reactive approach in healthcare must be transformed to a proactive approach that empowers individuals to manage their wellness. In this view, the nutrition habits 
and the level of physical activity are key determinants of wellness and this view is consistent with the well established human weight dynamics model proposed in [65]. While these models view a human as a sophisticated metabolic machine, recent literature has highlighted the importance of integrating human behaviors when using such models for weight dynamics [1].

Understanding and explaining the dynamics of human behavior is a challenging problem. For over five decades, psychologists, sociologists, and other researchers have developed several theories to explain and reason about human behavior. As examples, the Theory of Planned Behavior [10] and the Social Cognitive Theory [11] are two theories that have been widely used to address problems, such as smoking cessation, controlling the spread of epidemics, and other public health issues. Although these theories have been validated at the scale of the population, they are difficult to operationalize at the level of an individual. Recent efforts have focused on reducing the gap between what such frameworks offer and what is required to explain observed behavior in individual humans [66].

Recently, control theorists have offered more usable models that are based on the theories of human behavior change $[1,2,3,7,8]$. These models represent the constructs of the human behavior theories using a fluid flow analogy; here, a collection of tanks store fluid that flows from external sources and between the tanks in the system. These storage tanks represent latent factors, such as attitude, social norms and intention, that influence behavior from the point of view of a particular theory of human behavior. The rate of accumulation in a tank is the difference between the 
inflow into the tank and the outflow from the tank. The dynamic behavior of such a system is characterized by observing how the level of fluids in the tanks change over time. Despite the utility of these fluid analogy models in making the theories of behavioral change more accessible and operational, there can be some ambiguities in such models, especially when they are used to represent the complex energy transfers that occur within the human body.

Bond Graphs represent a domain independent modeling approach based on energy transfer between system components that captures the dynamic behavior of physical systems. When applied to human behavioral modeling, human behaviors can be interpreted as complex sequences of energy exchanges both within the human and between the human and their environment. For example, the digestive system transforms nutrition to biochemical energy stored in the body; this energy is used to effect mechanical action governed by several factors such as emotion, intent and self-efficacy. Finally, there is a source of potential ambiguity in the fluid analogy models; for example, the flow of fluid between interconnected tanks may or may not depend on the elevation of the tanks. For these reasons, the need to explore how Bond Graphs could be used to model and reason about the dynamics of human behavior change arises.

In this Chapter, Bond Graph models that capture the same behaviors as the fluid analogy models for human behavior change are developed. It is also shown that, Bond Graph models carefully address the problem of different tank elevations because of the natural feedback feature. In addition, this Chapter shows that, causality 
analysis can be used to validate the correctness of the model. Moreover it is also demonstrated that the Bond Graph models offer a comprehensive framework for representing and reasoning about the dynamics of human behavior change.

\subsection{Models for Human Behavioral Change}

Several theories and frameworks have been developed by psychologists and social scientists to explain human behavior at multiple scales. Some of the major theories that have been proposed include:

1. Theory of Planned Behavior (TPB) [10],

2. Social Cognitive Theory (SCT) [11],

3. Self Determination Theory (SDT) [12],

4. Transtheoretical Model (TTM) also known as the Stages of Change Model [13],

5. Theory of Reasoned Action (TRA) [67], and

6. Health Belief Model (HBM) [14].

Each of these theories propose a set of constructs and processes, to explain how they relate to human behavior while accounting for both environmental and individual influences. While these theories have been used to explain human behaviors at an aggregate level, they cannot be used as models that can be readily manipulated to offer interventions to individuals; because they were proposed as hypotheses for human behaviors. 


\subsubsection{Fluid Analogy Models}

Recently, control theorists have offered a new approach to operationalize some of the above theories. They have proposed fluid analogy models to represent and reason

about human behavioral change $[1,2,3,7,8]$. These models are based on some of the above well-established theories. The main objective in these fluid analogy models was to provide easy to understand, actionable, models that could be incorporated into systems that interact with humans. Two specific examples that were reported in the literature are described below for completeness.

The fluid analogy model shown in Figure 3.1 is the model shown in [2]. The constructs in TPB are Attitude, Social Norms, Intention, Perceived Behavioral Control and Behavior. As illustrated, these constructs are represented as storage tanks. Valves and pipes are used to link the interactions between the constructs. External influences are represented as sources in the model. Model parameters are included to capture and represent variations between individuals. 


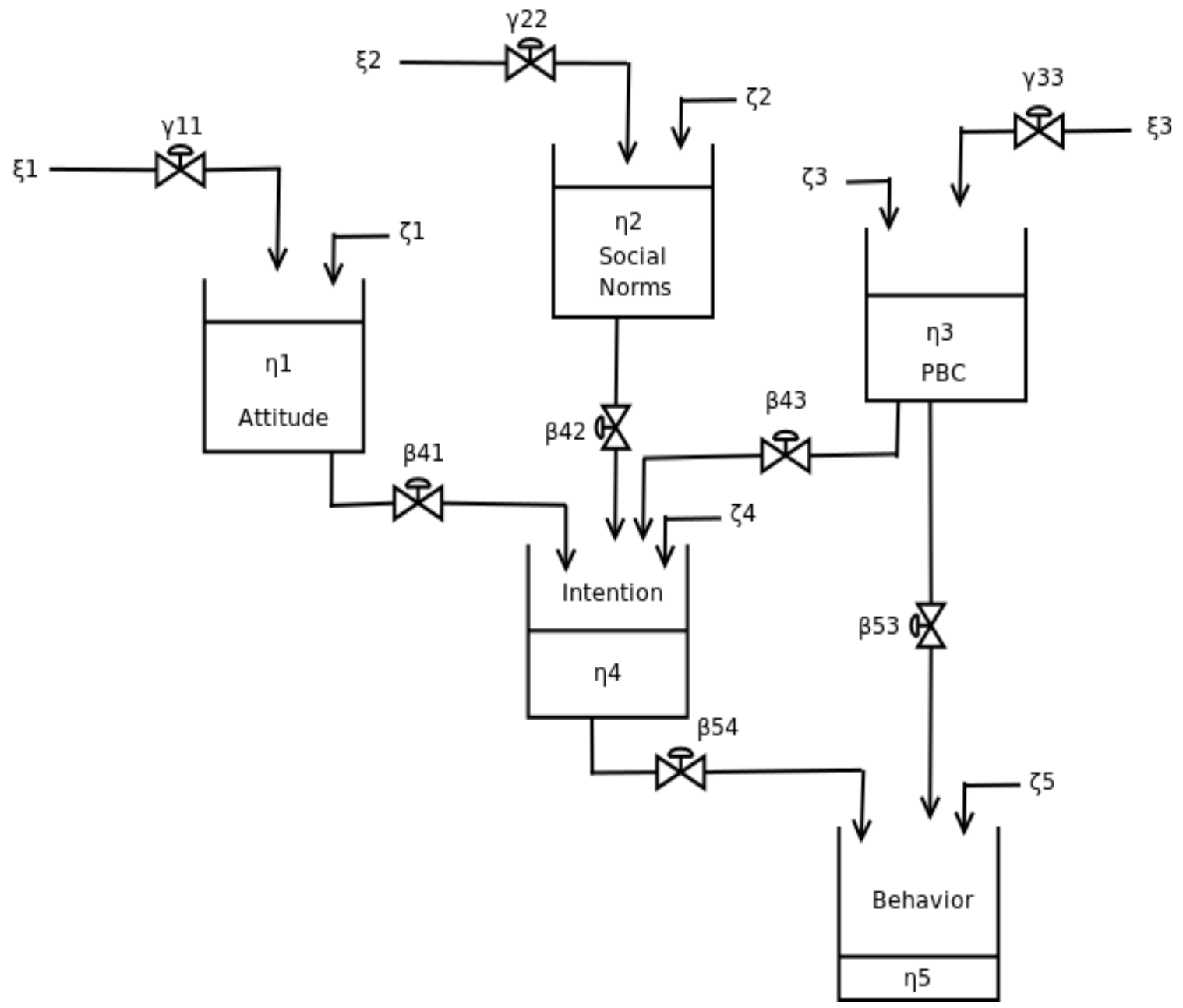

Figure 3.1: Fluid analogy model based on the Theory of Planned Behavior as presented in $[1,2]$.

The dynamic equations that represent the behavior of the system were derived using the principle of conservation of mass, i.e., accumulation in a tank is the difference between the inflow to the tank and the outflow from the tank.

Another fluid analogy model that is based on a Simplified Social Cognitive Theory as reported in [7] is shown in Figure 3.2. The constructs in the simplified SCT are Behavior, Behavior Outcomes, Cues to Action, and Outcome Expectancy. 
As discussed in the preceding model, tanks are associated with these constructs. In this case, there is a reinforcement pathway where the behavior reinforces the behavior outcomes.

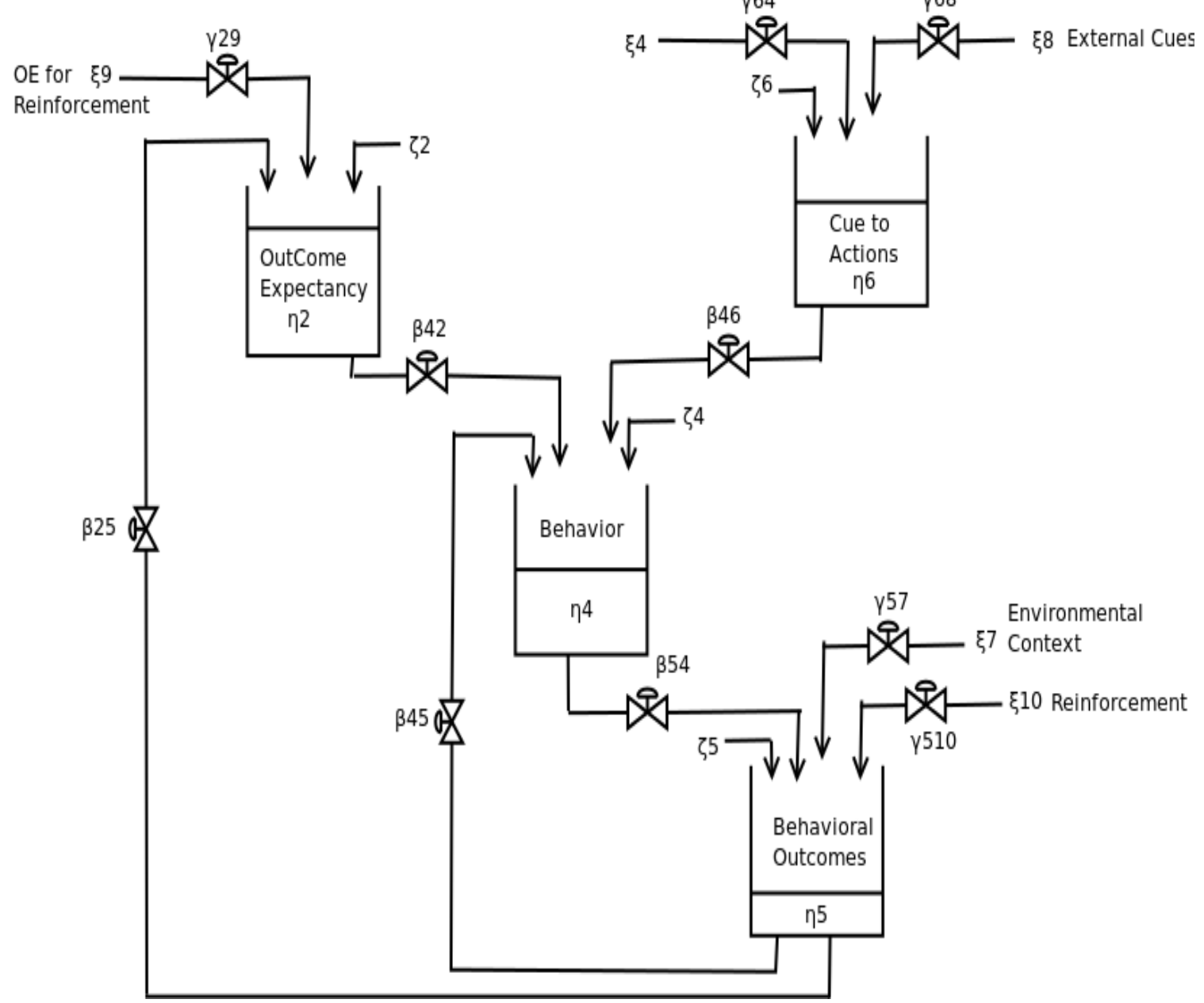

Figure 3.2: Fluid analogy of the simplified Social Cognitive Theory as presented in $[3]$.

\subsubsection{Ambiguity in the Fluid Analogy Models}

The fluid analogy models can, however, be ambiguous. Consider the fluid analogy model based on the Theory of Planned Behavior shown in Figure 3.1 as presented 
in $[1,2]$. From these fluid flow models, it is not clear if the relative elevation of the tanks affect the flow. For example, in Figure 3.1, social norms affect intention and not vice versa. Similarly, attitude affects intention and not vice versa. Are these constructs of TPB indeed asymmetrical? Further, the flow from Behavioral Outcomes to Behavior in Figure 3.2 indicates that the valve $\beta 45$ is a control valve. Since the notation of all the valves in Figure 3.1 and Figure 3.2 are the same, it can be assumed that the valves are all control valves. However, neither the representation of the fluid analogy models nor the dynamic equations derived from these models in $[1,2,3]$ show the energy that is required to operate the control valves. On the other hand, the bond graph models do not have such ambiguities.

Consider the two tank model shown in Figure 2.2. Notice that the change of fluid level in $h_{1}$ is affected by the level $h_{2}$ when $R$ is a conventional valve as demonstrated in Equation (2.1). On the other hand, if $R$ is a controlled, one-directional valve, that requires additional energy to cause the flow, the bond graph model is as illustrated in Figure 3.3. The external modulator changes the value of the resistor based on the environmental conditions. 


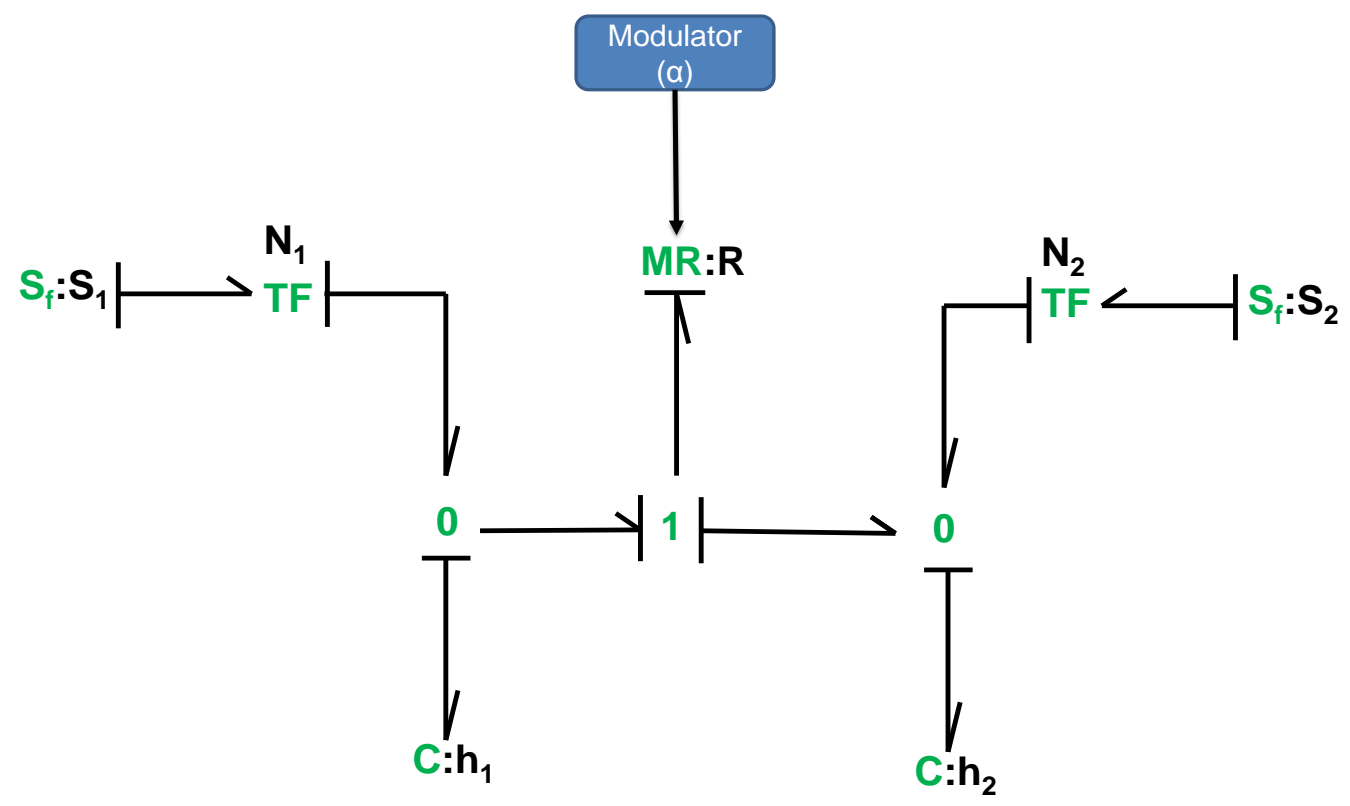

Figure 3.3: Bond graph model for the two tank system assuming that $R$ is a Control Valve.

The dynamic equations that are derived from the bond graph in Figure 3.3

are

$$
\begin{aligned}
\frac{d h_{1}}{d t} & =\frac{S_{1} N_{1}}{\rho g C_{1}}-\frac{1}{R^{\prime}}\left(h_{1}(t)-h_{2}(t)\right), \\
\frac{d h_{2}}{d t} & =\frac{S_{2} N_{2}}{\rho g C_{2}}+\frac{1}{R^{\prime}}\left(h_{1}(t)-h_{2}(t)\right) .
\end{aligned}
$$

Note that $R^{\prime}$ is not a constant value and depends on the modulation signal. Equation (2.1) describes the dynamic behavior of the two tank system assuming that 
$R$ is a conventional valve. Note that this model accounts for the level of fluid in both the tanks. The Equation (3.1) represents the dynamic behavior that were derived from the bond graph model shown in Figure 3.3 when $R^{\prime}$ is a control valve. Thus, the ambiguity of the type of valve is resolved because the bond graph model shows the external modulator and the dynamic equations use an externally modulated value for the resistance $\left(R^{\prime}\right)$.

An important aspect to notice in the bond graph models is that there are natural feedback loops in human behaviors. Such feedback loops are indeed captured in the bond graph models. For example, in Figure 4.8 notice that there is a natural feedback loop between attitude and intention, and between social norms and intention. In the event these constructs are asymmetric and decoupled, then the bond graph model would utilize modulated resistors in place $\beta_{41}$ ane $\beta_{42}$. In contrast, such loops must be explicitly accounted for in the fluid analogy models by changing the either the valve that regulates the flow between two tanks, or by changing the design of the pipes. Special attention must be paid to whether or not gravity affects the dynamics of the system.

From the discussion in this section, it can be concluded that, fluid analogy models for human behavior have been very useful to make the theories of human behavior more accessible and actionable. The tank analogies which represent internal factors that influence behavior are intuitive. The connections between the tanks and the control parameters are convenient for specializing the models for individuals. There is, however, a source of ambiguity in the fluid analogy models and these 
ambiguities are resolved in the bond graph models. These ambiguities do not arise because causality analysis forces the designer to explicitly represent all the sources of energy and energy transfers in the system.

\subsection{Bond Graphs Models for Human Behavior Change}

This section presents bond graph models for human behavior using the well-developed fluid analogy models as a starting point. This approach allows the reuse of the control parameters. The focus is on the Theory of Planned Behavior and Social Cognitive Theory. Using the fluid analogy models as a starting point, bond graphs were constructed, carried out causality analysis and derived dynamic equations from these models. The remainder of this section presents the models and the dynamic equations.

\subsubsection{Theory of Planned Behavior}

The storage tanks shown in Figure 3.1, i.e., Attitude $\left(\eta_{1}\right)$, Social Norms $\left(\eta_{2}\right)$, Perceived Behavioral Control $\left(\eta_{3}\right)$ (PBC), Intention $\left(\eta_{4}\right)$ and Behavior $\left(\eta_{5}\right)$, are represented as storage elements of type $C$ in the bond graph model shown in Figure 4.8. In addition to these state variables, the input sources are Behavioral Belief $\left(\xi_{1}\right)$, Normative Belief $\left(\xi_{2}\right)$ and Control Belief $\left(\xi_{1}\right)$; these are modeled as sources of flow in the bond graph $S_{f}$. The uncertainties associated with the state variables are modeled as disturbances (illustrated as sources of flow), $\varsigma_{1}, \varsigma_{2}, \cdots$, and $\varsigma_{5}$, in the bond graph model. 
Causality analysis was carried out by placing the strokes on the bonds to represent the flow in the system. Comparing with the fluid flow illustrated in Figure 3.1, notice that the causality assigned corresponds to these flows. For example, note that the levels of Attitude and Social Norms affect level of intention; the level of PBC affects both intention and behavior. The main idea of TPB, i.e., intention directly affects behavior, is also evident in the bond graph model.

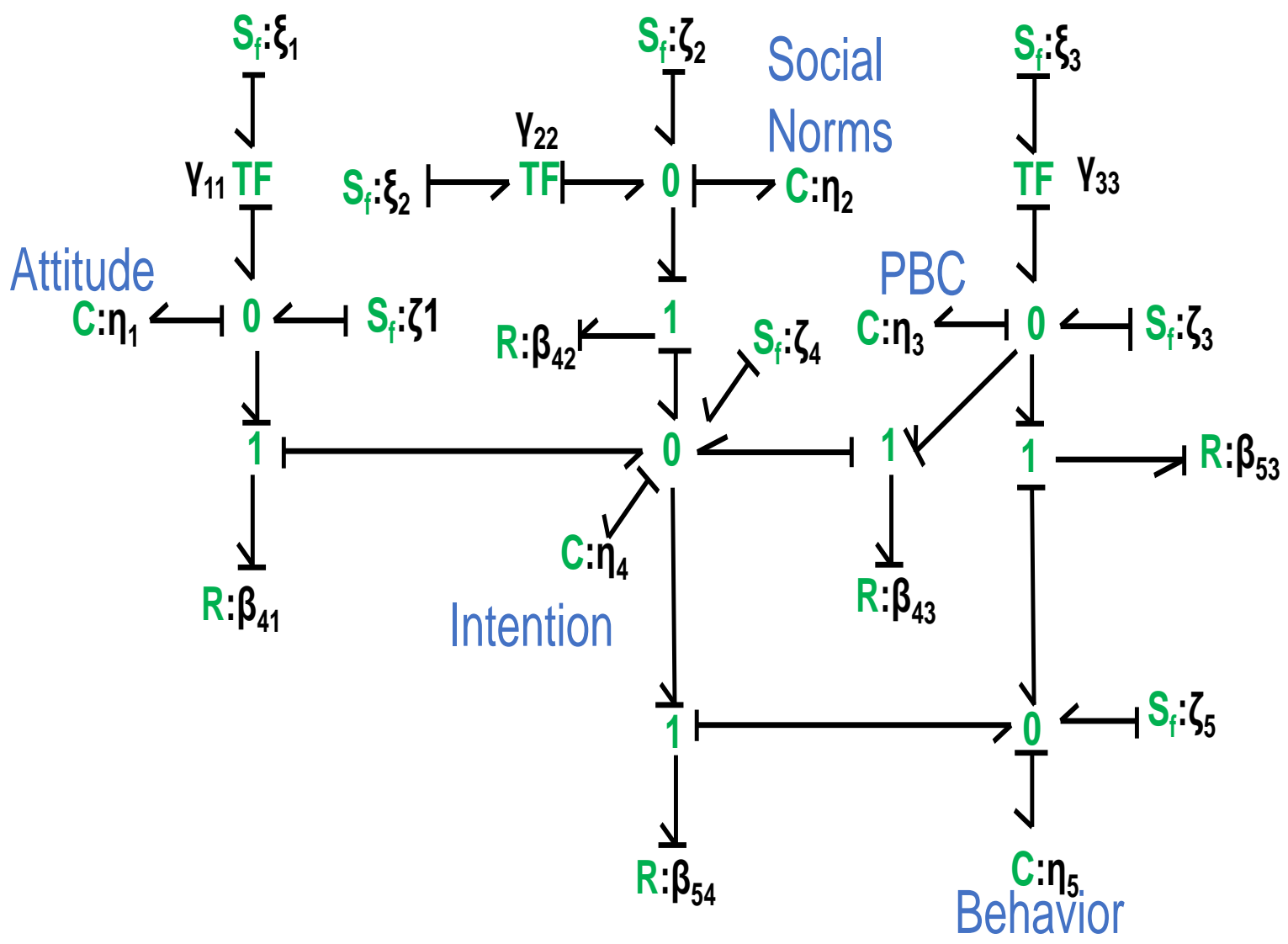

Figure 3.4: Bond graph model based on the Theory of Planned Behavior. Note, the sources of flow $\zeta_{i}$ represent disturbances. 
Dynamic equations obtained from the bond graph are:

$$
\begin{aligned}
& \frac{d \eta_{1}}{d t}=\gamma_{11} \xi_{1}-\frac{1}{\beta_{41}}\left(\eta_{1}-\eta_{4}\right)+\varsigma_{1} \\
& \frac{d \eta_{2}}{d t}=\gamma_{22} \xi_{2}-\frac{1}{\beta_{42}}\left(\eta_{2}-\eta_{4}\right)+\varsigma_{2} \\
& \frac{d \eta_{3}}{d t}=\gamma_{33} \xi_{3}-\frac{1}{\beta_{43}}\left(\eta_{3}-\eta_{4}\right)-\frac{1}{\beta_{53}}\left(\eta_{3}-\eta_{5}\right)+\varsigma_{3} \\
& \frac{d \eta_{4}}{d t}=\frac{1}{\beta_{41}}\left(\eta_{1}-\eta_{4}\right)+\frac{1}{\beta_{42}}\left(\eta_{2}-\eta_{4}\right)+\frac{1}{\beta_{43}}\left(\eta_{3}-\eta_{4}\right)-\frac{1}{\beta_{54}}\left(\eta_{4}-\eta_{5}\right)+\varsigma_{4} \\
& \frac{d \eta_{5}}{d t}=\frac{1}{\beta_{53}}\left(\eta_{3}-\eta_{5}\right)+\frac{1}{\beta_{54}}\left(\eta_{4}-\eta_{5}\right)+\varsigma_{5}
\end{aligned}
$$

\subsubsection{Simplified Social Cognitive Theory}

The inputs in the simplified Social Cognitive Theory model are Internal Cues $\left(\xi_{4}\right)$, External Cues $\left(\xi_{8}\right)$, Environmental Context $\left(\xi_{7}\right)$, Outcome Expectancy Reinforcement $\left(\xi_{9}\right)$ and Reinforcement $\left(\xi_{10}\right)$. 
The internal cues and external cues affect the level of the storage element Cues to Action $\left(\eta_{6}\right)$. The Behavioral Outcomes are affected by Outcome Expectancy, Environmental Context and Reinforcement. The level of Behavior $\left(\eta_{4}\right)$ is affected by the levels of the other three state variables.

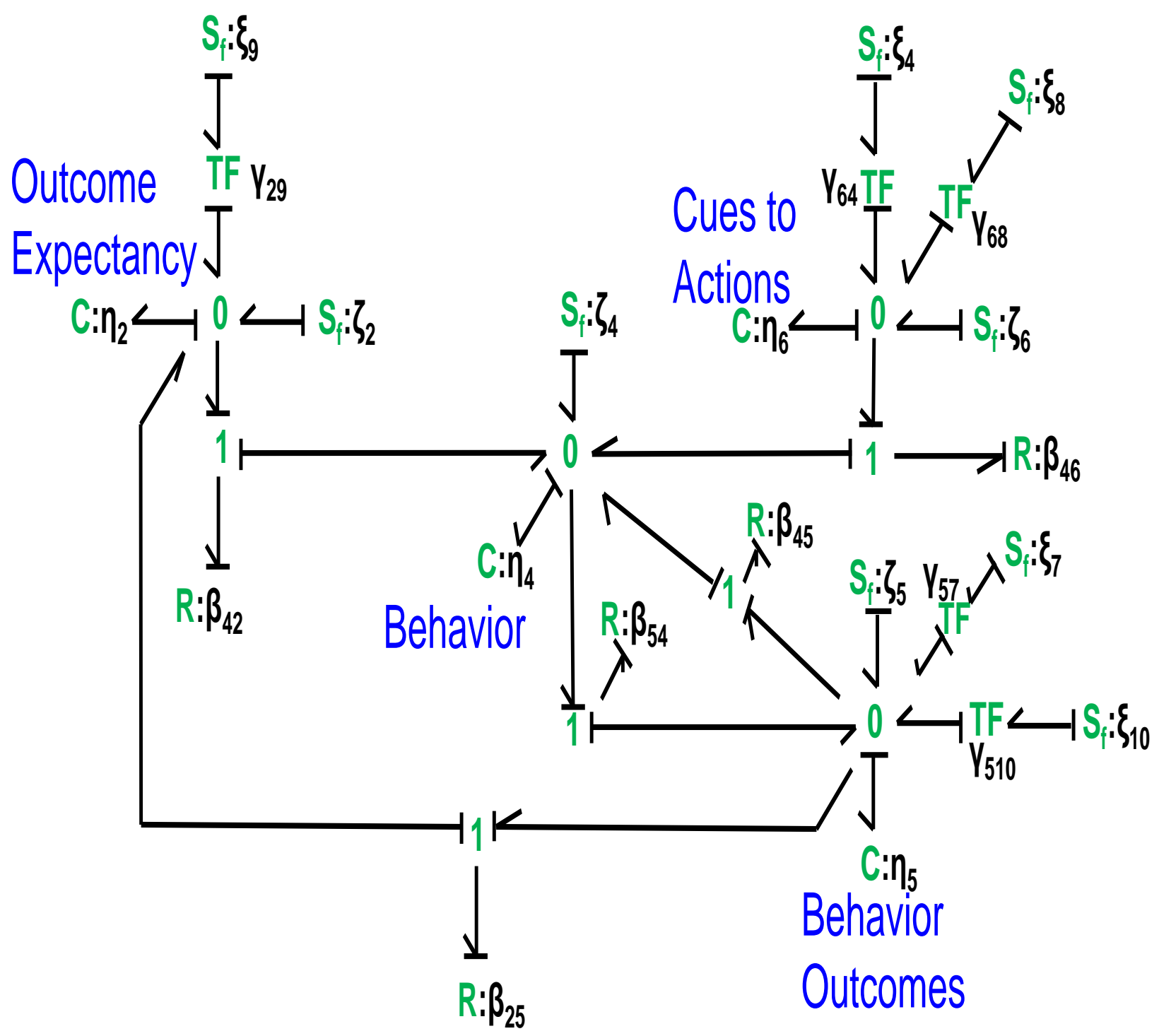

Figure 3.5: Bond graph model based on the simplified Social Cognitive Theory. 
The dynamic equations obtained from the bond graph are:

$$
\frac{d \eta_{2}}{d t}=\gamma_{29} \xi_{9}+\frac{1}{\beta_{25}}\left(\eta_{5}-\eta_{2}\right)-\frac{1}{\beta_{42}}\left(\eta_{2}-\eta_{4}\right)+\varsigma_{2}
$$

$$
\frac{d \eta_{4}}{d t}=\frac{1}{\beta_{46}}\left(\eta_{6}-\eta_{4}\right)+\frac{1}{\beta_{42}}\left(\eta_{2}-\eta_{4}\right)+\frac{1}{\beta_{45}}\left(\eta_{5}-\eta_{4}\right)-\frac{1}{\beta_{54}}\left(\eta_{4}-\eta_{5}\right)+\varsigma_{4}
$$

$$
\frac{d \eta_{5}}{d t}=\gamma_{57} \xi_{7}+\gamma_{510} \xi_{10}+\frac{1}{\beta_{54}}\left(\eta_{4}-\eta_{5}\right)-\frac{1}{\beta_{45}}\left(\eta_{5}-\eta_{4}\right)-\frac{1}{\beta_{25}}\left(\eta_{5}-\eta_{2}\right)+\varsigma_{5}
$$

$$
\frac{d \eta_{6}}{d t}=\gamma_{64} \xi_{4}+\gamma_{68} \xi_{8}-\frac{1}{\beta_{46}}\left(\eta_{6}-\eta_{4}\right)+\varsigma_{6}
$$

\subsection{System Analysis}

The dynamic equations of a system help to understand how the system will respond to changes in the inputs. This formulation allows the abstraction of the human behavior, the analysis of the behaviors, and the design of the controllers that can help achieving the desired behaviors. Of course, it is important not to forget that 
these control actions in fact affect humans and hence mechanisms that ensure that the control actions are effectively conveyed to the humans and adopted by them to realize the changes in behavior must be designed.

In order to systematically analyze the dynamic behavior represented by the equations in the preceding section, reasonable values were selected for the constants to emphasize the relationships between the inputs and outputs. The impulse response was then examined and step response of the system. The transfer function was generated and evaluated the stability of the system.

\subsubsection{Time response}

There are five state variables and three inputs in the bond graph for the TPB system. Each state variable was associated with a disturbance; a normal distribution with mean value 0 and variance 0.1 was assigned. All the transformer ratios were assigned a value 1 for simplicity. To regulate the flows, the values of $\beta_{41}=0.8, \beta_{42}=0.5$, $\beta_{43}=0.4, \beta_{53}=0.4$, and $\beta_{54}=0.9$ were selected

Figure 3.6 illustrates how the state variables change in response to a step input (top) and a sinusoidal input (bottom). For the step input, all the three inputs, i.e., Behavioral Belief, Normative Belief, and Control Belief, were assigned a step value 1 and for the sinusoidal inputs, all the inputs were assigned value $\sin (t)$ at time $t$.

Notice that as time progresses the levels of the state variables increase linearly in response to a step input. Attitude, Social Norms and PBC have higher 
values because they are directly connected to the inputs. The value of Social Norms is high because $\beta_{42}=0.5$ and hence the flow out of this storage variable is slower. Behavior follows Intention closely both in response to the step input and in response to the sinusoidal input.

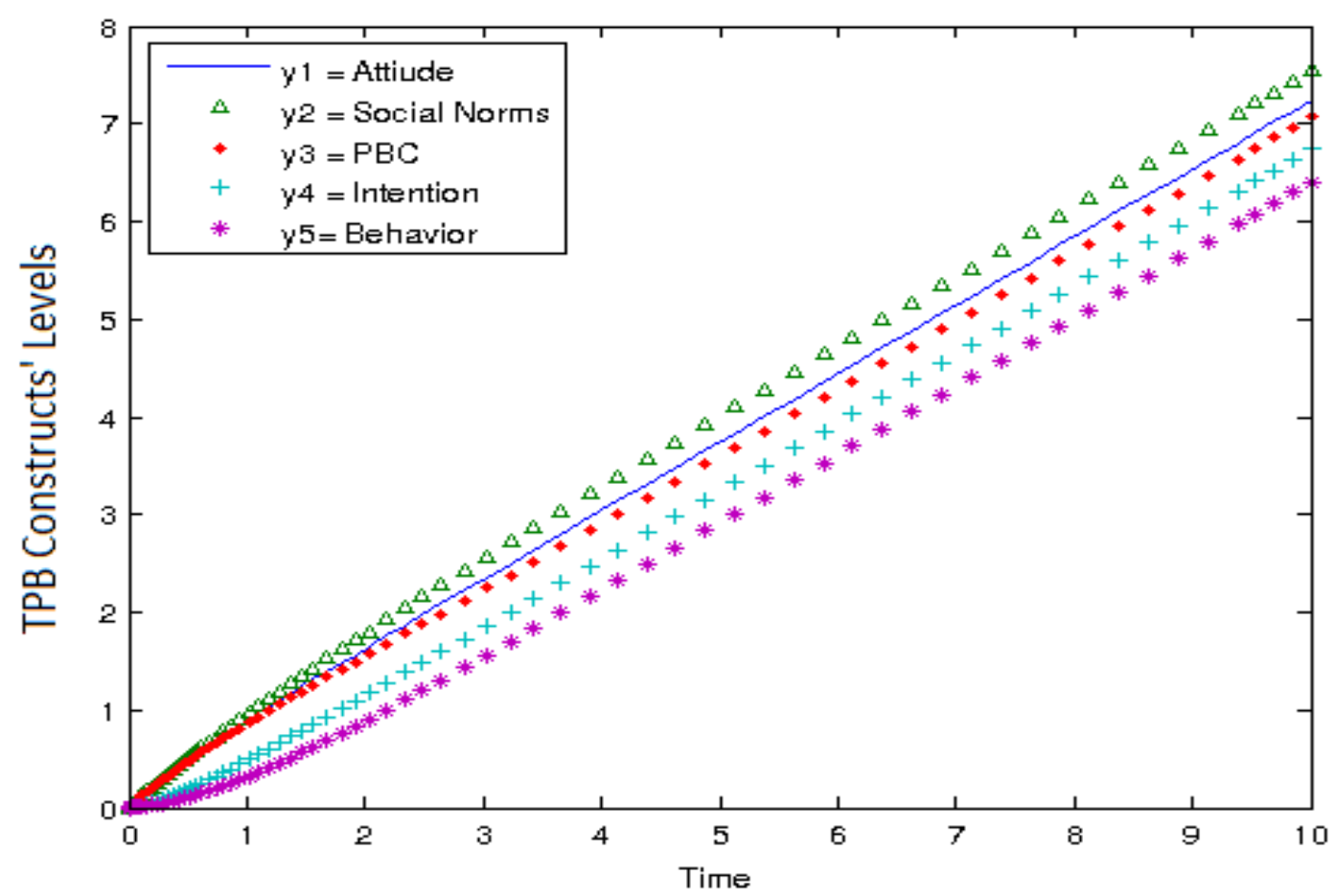

Figure 3.6: Values of the state variables in response to a step input of 1 at all the three inputs. Notice that Behavior is most closely related to Intention as can be noticed from the bond graph model in Figure 4.8. 


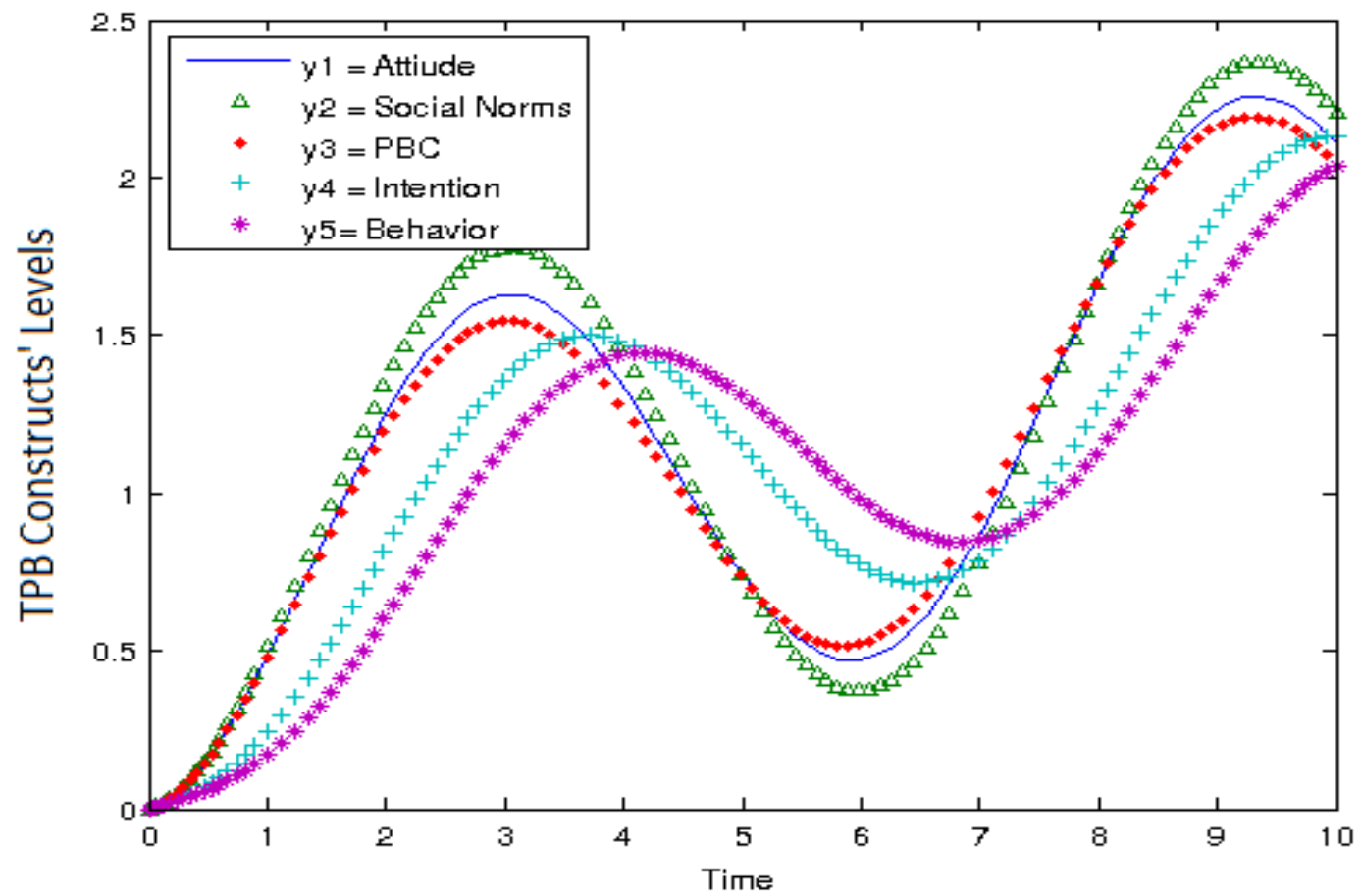

Figure 3.7: Values of the state variables when the inputs are sinusoidal, the response is different. Notice that Behavior is most closely related to Intention as can be noticed from the bond graph model in Figure 4.8.

In practice, if the values for the resistors and the inputs accurately for an individual can be estimated, it is now possible to use this approach to predict what future behavior can be expected. This understanding may be valuable to offer suitable interventions by designing controllers.

\subsubsection{Stability}

In [68], it was showed that the two tank model is a convenient model for the interaction between a client and a therapist. Analogous to the ideas in [16], the fluid levels of the tanks represented the valence, or affect, of the client $\left(C_{2}\right)$ and the therapist 
$\left(C_{1}\right)$. The model shown in Equations 4.1 was used to analyze the stability of the system. For example, the critical points were identified and the system trajectories were plotted starting from different initial conditions, i.e., different initial states of both the client and the therapist. For example, if the therapist has initially positive valence, how is that going to affect the initially negative, neutral or positive client. Using the parameters from [16] the phase portraits shown in Figure 4.6 were obtained

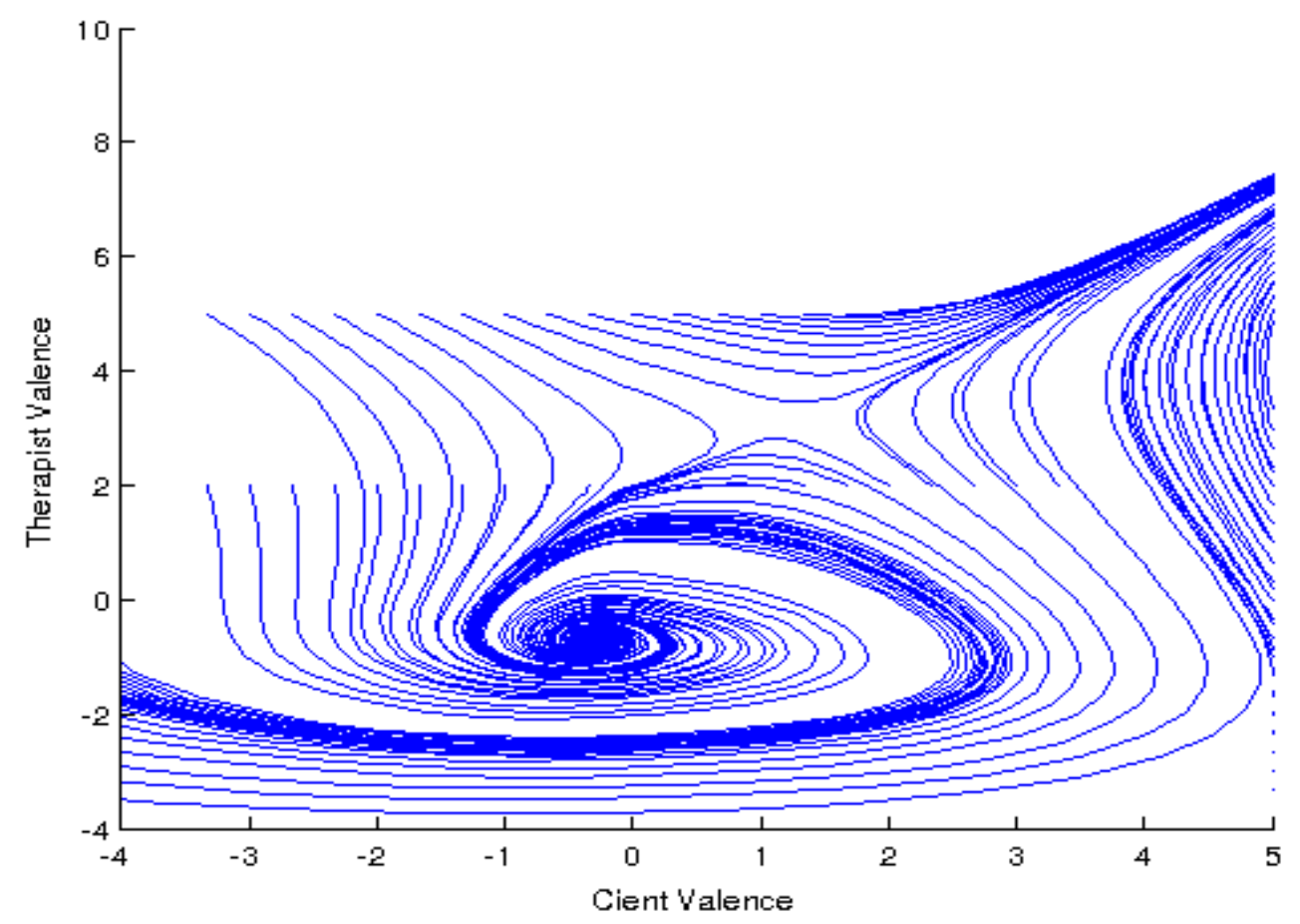

Figure 3.8: The phase portraits for the psychotherapy relation

Notice from Figure 4.6 that there are two critical points that must be observed - the first is a stable point or Attractor at the location $(-0.4,0)$ as indicated 
on the scale. This is a desirable point that must be reached as a conclusion of a successful therapy since it moves the client from one equilibrium to another. The other critical point to observe is the saddle point that appears on the top right part of the figure; this represents a failed therapy. The figure shows that, when the therapist has a positive valence, the therapy sessions can lead a client with a negative or positive valence to the attractor. On the other hand a when the therapist has a negative valence, the session may fail.

\subsection{Evaluation}

To validate the bond graph models, three scenarios for a hypothetical 25 year old male participant were considered, Tom, who weighs $112 \mathrm{Kg}$ (246 lbs). Suppose that Tom is seeking interventions to help with weight loss.

The well-established weight dynamics model proposed by Hall was used as a reference [65]. This model accepts as input the physical activity level and nutrition level of the participant in intervals of one day; the output of the model is a predicted trajectory for the weight of the participant over a period of up to 180 days. To use this model, a nutrition and physical activity level profile were created for Tom in three different scenarios.

In the first scenario, it is assumed that Tom consumed 2200 calories per day and expends about 400 calories per day in addition to his basal metabolic rate through focused exercise activities. Using this baseline scenario, the weight dynamics model was used to compute the weight trajectory for Tom for 180 days. To evaluate 
the effectiveness of these human behavior models, it is assumed in Scenario 2 that Tom seeks intervention and is resistant to follow the advice. In Scenario 3, it is assumed that Tom seeks intervention and diligently follows the advice offered. Both the TPB model and the simplified SCT model were used to investigate how Tom's behavior, i.e., adherence to the nutrition and physical activity recommendations, affect his weight dynamics over the same period of 180 days.

It is assumed that without any intervention, i.e., in Scenario 1, that variation in the nutrition because of uncertainties is between 1500 kCalories and 2900 kCalories. Similarly, it is assumed that the calories expended because of exercise were between 330 calories and 650 calories. Under these assumptions, the baseline weight dynamics for a duration of 180 days are illustrated in Figure 3.9. The solid line in the middle of the figure on the left illustrates the weight when there is no uncertainty in the nutrition intake or the physical activity level. The two dotted lines, above and below the solid line, illustrate the variation in the weight that is possible if the uncertainties occur as indicated.

\subsubsection{TPB Bond Graph Model}

The weight dynamics for Scenario 2 and Scenario 3 were computed using the TPB Bond Graph model. Here, it is assumed that the intervention only affects the participant's perceived behavioral control, i.e., input $\zeta_{3}$. It is also assumed that the social norms are constant, i.e., $\zeta_{2}=-0.5$, and Attitude remained constant, i.e., $\zeta_{1}=0.5$. The results are shown in Figure 3.9. The line with the stars represents the weight dy- 
namics in Scenario 2. Recall, in this scenario, the participant is resistant to change. For this reason, over the duration of 180 days, he only reduces about $3 \mathrm{Kg}$. The line with the circles illustrates the weight dynamics in Scenario 3. Notice in this case that the change achieved is very close to the maximum that can be achieved according to the Hall model.

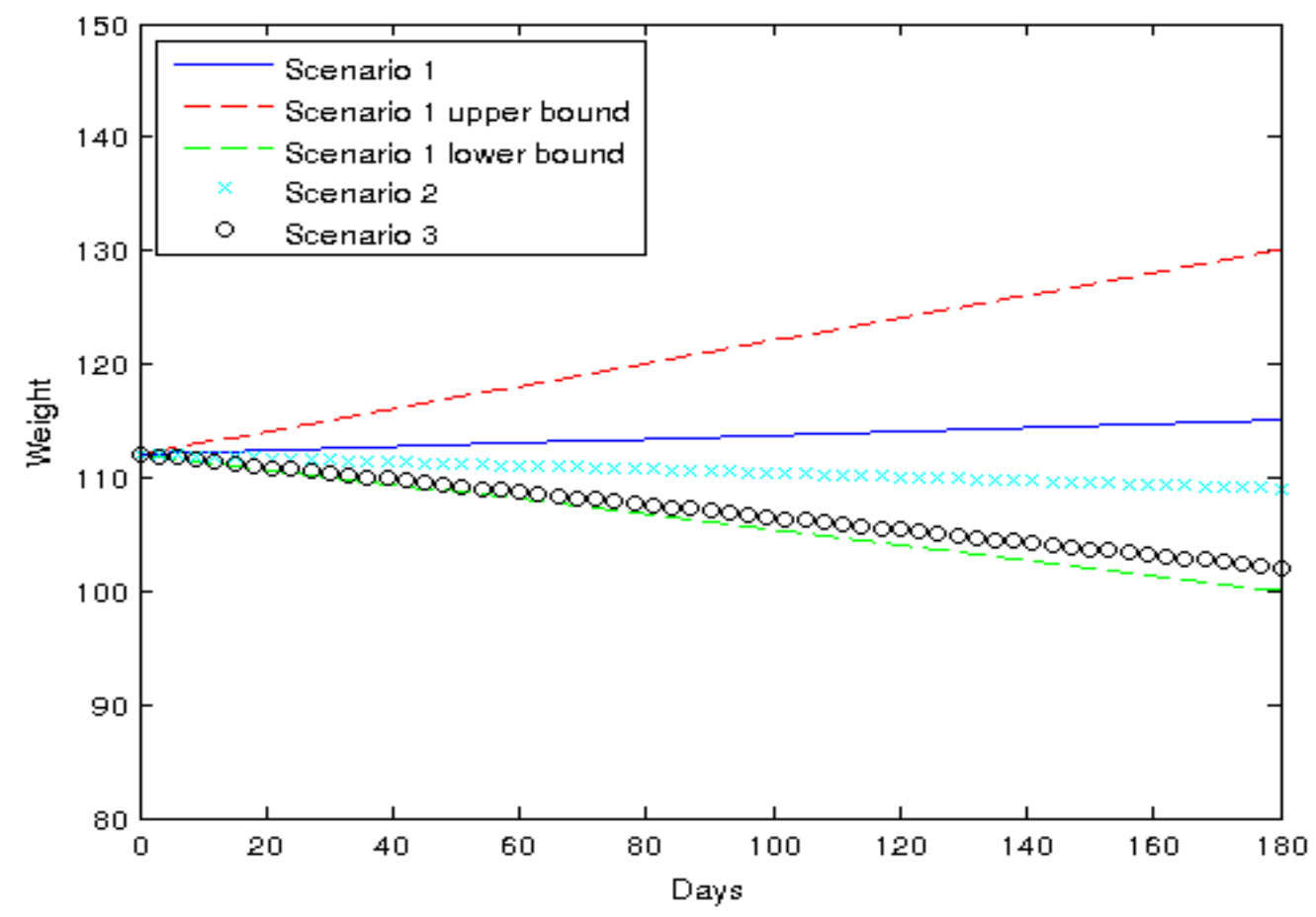

Figure 3.9: The weight dynamics for the participant in three scenarios. Scenario 1 is the baseline. The participant is resistant to change in Scenario 2 and the participant diligently complies with the recommendations in Scenario 3 as computed using the TPB bond graph model.

To understand the dynamics of the TPB model, it is important to examine how perceived behavior control (PBC) affects both the intention and the behavior of the participant. In Scenario 2 the resistance value associated with PBC was high, 
i.e., the valve was close $80 \%$. In Scenario 3, this valve was closed only $20 \%$ and the resistance was low. Figure 3.10 illustrates the change in state variables of the TPB model over the duration of 180 days. The top part of the figure illustrates the values of these variables for Scenario 3 and the bottom part illustrates corresponding values for Scenario 2. Notice that because of the high resistance in Scenario 2, the behavior is correspondingly undesirable and the outcomes, i.e., weight loss, is low.

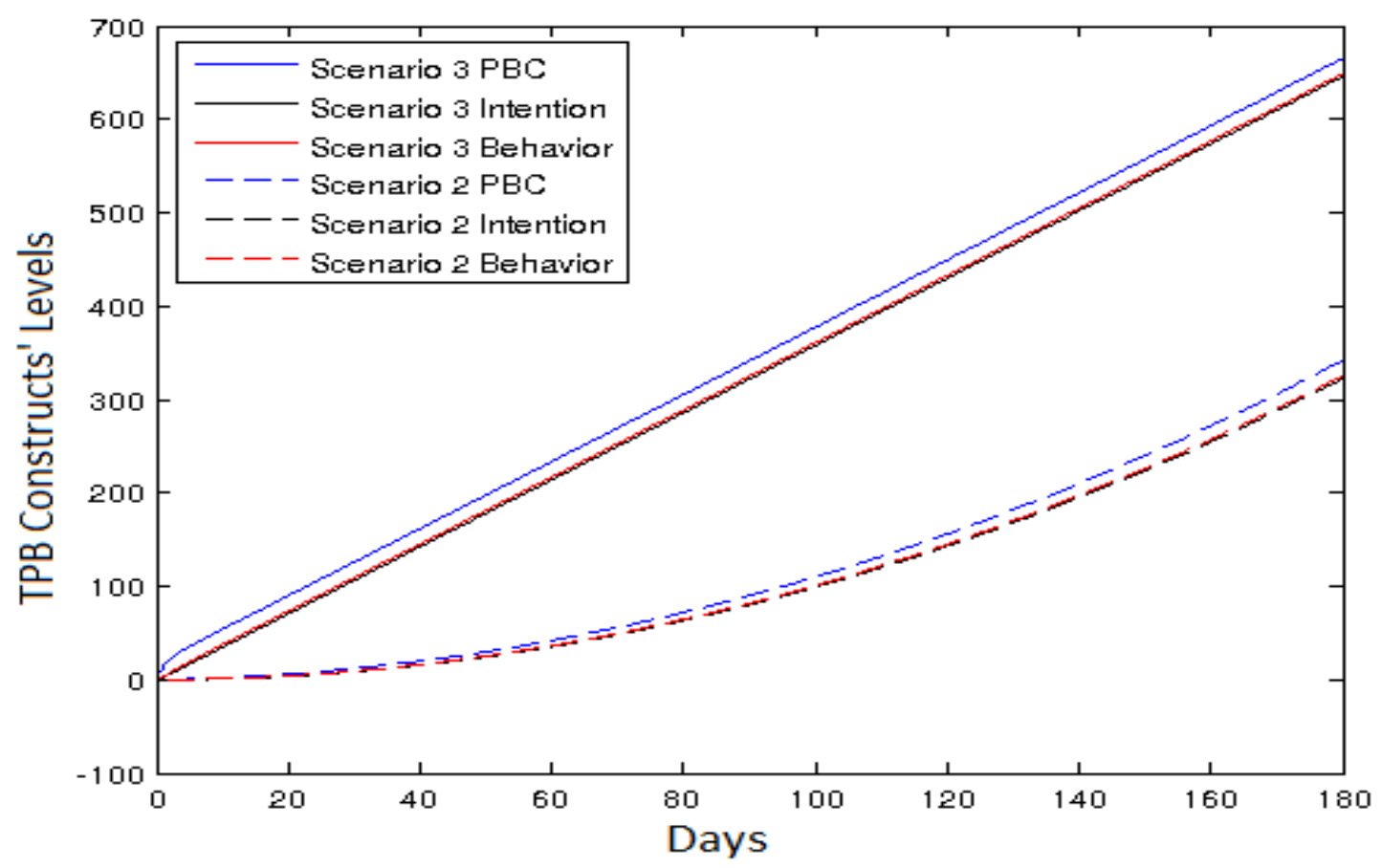

Figure 3.10: When the resistance to PBC intervention is low, the participant responds promptly (top), behavior improves and the weight loss is achieved as desired. When the resistance is high (bottom), the behavior is undesirable and weight loss is not achieved. 


\subsubsection{SCT Bond Graph Model}

Figure 3.11 and Figure 3.12 present similar results obtained using the SCT Bond Graph model to compute the interventions. In this model, the external cues to action was used as the intervention. As illustrated in Figure 3.11, in Scenario 2 the weight loss is about $5 \mathrm{Kg}$ because the participant's resistance to intervention is high, i.e., valve was closed to $80 \%$. Note that in Scenario 3, the weight loss matches the best that can be achieved according to the weight dynamics model. Figure 3.12 shows how the change in the external cues to action affects behavior in Scenario 2 and Scenario 3. 


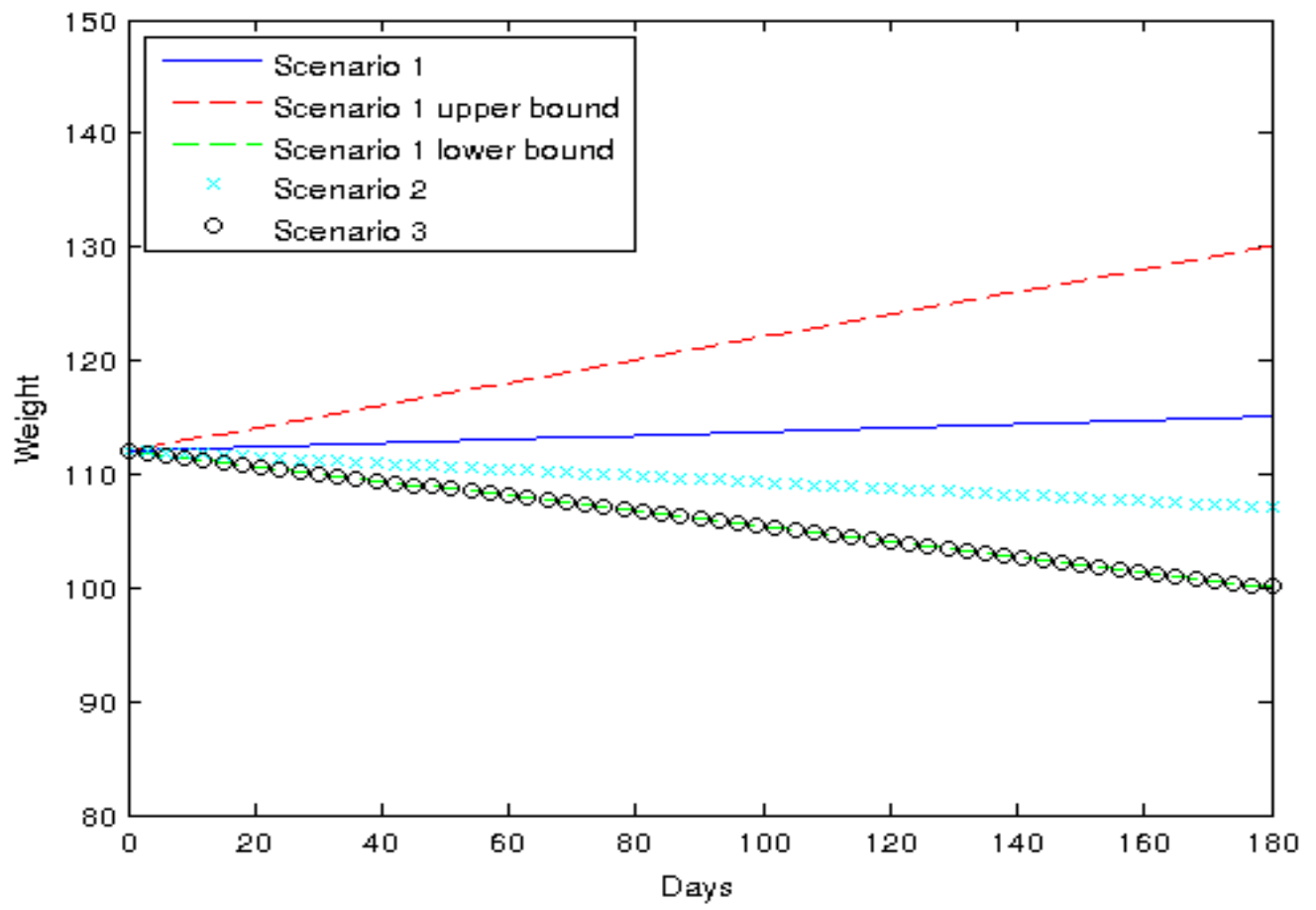

Figure 3.11: The weight dynamics for the participant in three scenarios. Scenario 1 is the baseline. The participant is resistant to change in Scenario 2 and the participant diligently complies with the recommendations in Scenario 3 as computed using the SCT bond graph model. 


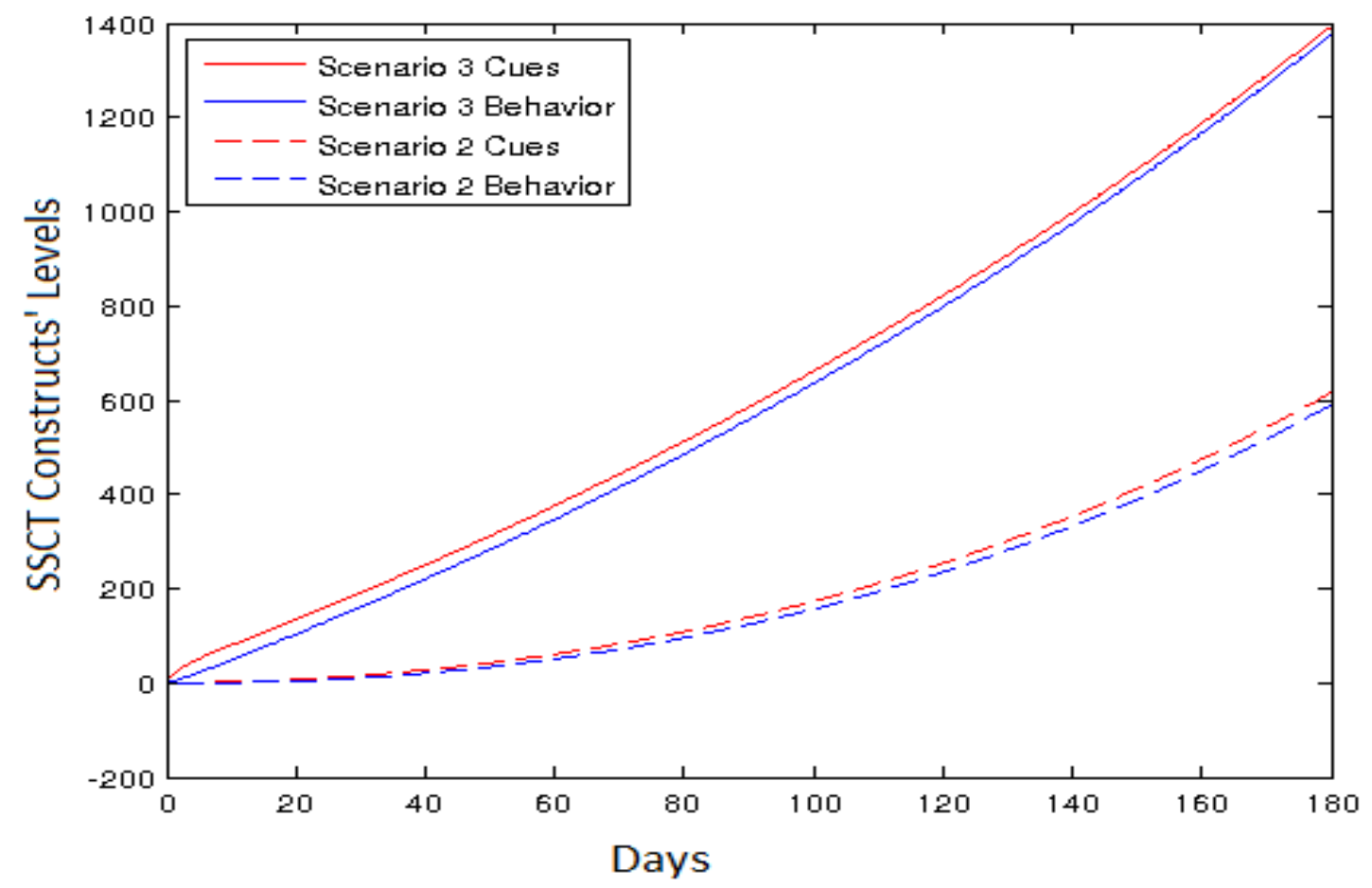

Figure 3.12: When the resistance to external cues intervention is low, the participant responds promptly (top), behavior improves and the weight loss is achieved as desired. When the resistance is high (bottom), the behavior is undesirable and weight loss is not achieved.

\subsection{Discussion}

The presented Bond Graph models for human behavior are versatile and domain independent. The results in this section demonstrate that both the TPB and SCT based Bond Graph Models are useful for providing weight loss interventions. The results show that the SCT model helps the hypothetical participant to achieve better weight loss. This is likely because of the reinforcement that happens in SCT via the feedback pathway that connects behavior outcomes with behavior. 
The causality analysis that can be carried out using the bond graph models is useful during model construction to better understand the system and verify correctness of the model. In addition, it helps to identify the state variables of the system. Further, causality analysis is also useful during diagnosis of the system. Through system identification, what interventions are necessary to help a participant achieve their target objectives can be determined.

These initial steps to model human behavior using bond graphs are promising. By organizing communities of people into groups that have similar behavior it may be possible in the future to construct behavior models at larger scales and identify interventions that can effectively improve the wellness of communities.

\subsubsection{Hybrid Bond Graph Models}

Challenges that result from discontinuities or abrupt changes in behavior can be better modeled and analyzed using Hybrid Bond Graphs [69]. The main idea here is to switch the dynamics of the system by modifying the states of the system based on the change that occurred. The dynamics are resumed in the new mode. Such mode switches are accomplished through Controlled Junctions. In addition to the model, the modeler must provide a control specification (CSPEC) that regulates the operations of the controlled junctions [69].

This Section presents an example to illustrate this idea. Consider the TPB model for an individual who has a low level of physical activity and poor nutrition. Suppose this individual receives a diagnosis that requires him to change his exercise 
and nutrition habits. This person may change from being well-motivated to becoming complacent several times before he settles down in a new routine. Such a system can be modeled using three modes of operation: 1) when the person is complacent and unmotivated, 2) instantaneous triggers (say interventions from a therapist) that cause the individual to switch from one mode to another, and 3) when the person is motivated to exercise and eat well.

In the first mode, the inputs to the system are stable with negative values for the Behavioral Belief $\left(\xi_{1}\right)$ and the Normative Belief $\left(\xi_{2}\right)$, and a small positive value for the Control Belief $\left(\xi_{1}\right)$. In the second mode, an impulse occurs in the Control Belief $\left(\xi_{1}\right)$, and in the third mode there is a large positive step in the Behavioral Belief $\left(\xi_{1}\right)$. This situation can be modeled as a bond graph with one controllable junction associated with the Behavioral belief input as illustrated in Figure 3.13. The CSPEC for this junction depends on a condition of the Control belief when the effort input cross a certain threshold. When the controllable junction is turned ON, the Behavioral input changes the state vector and pushes the system into the third mode. 


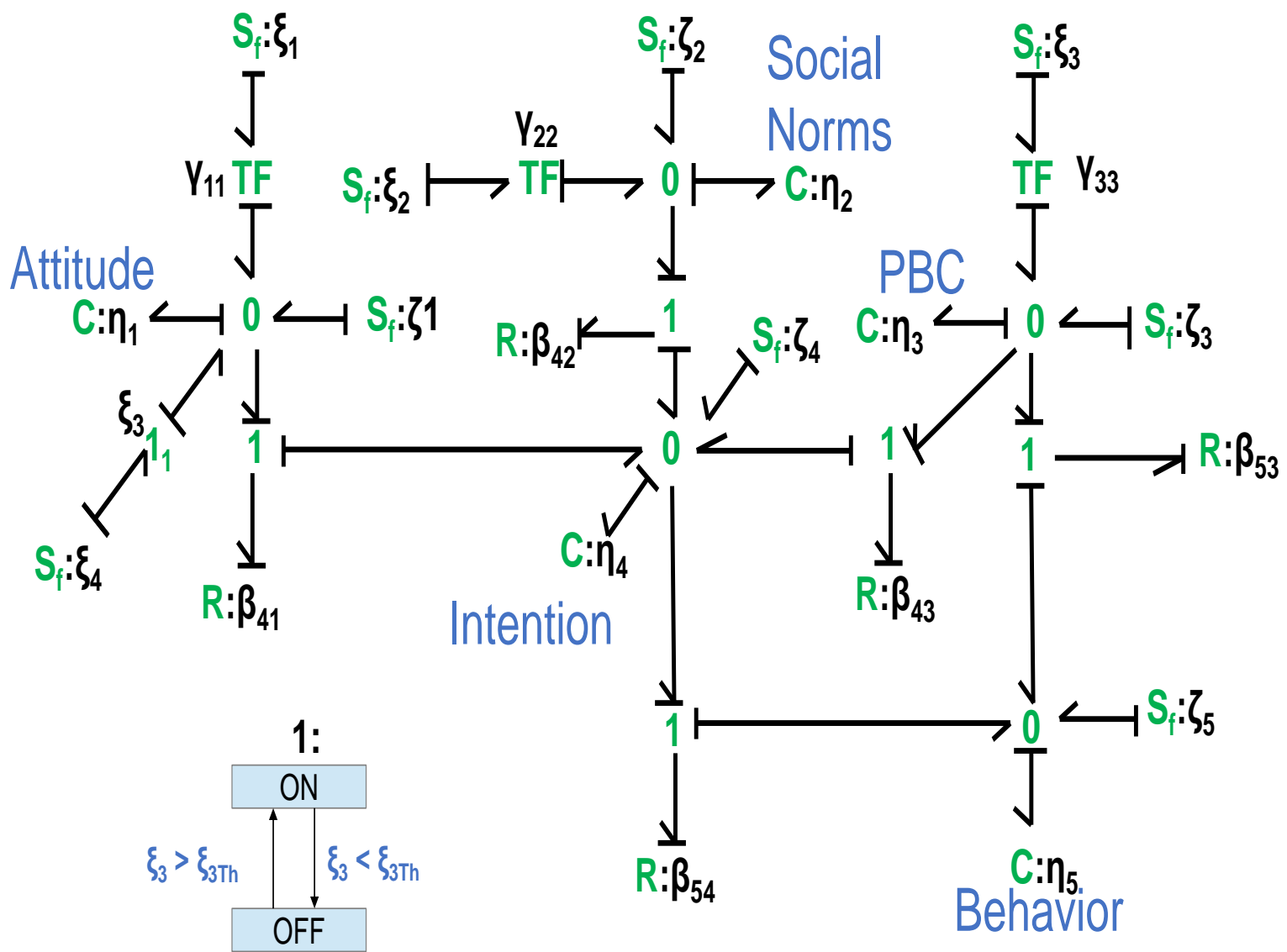

Figure 3.13: Hybrid bond graph model for the Theory of Planned Behavior.

A thorough analysis of such a model and a comprehensive review of the relevance of hybrid bond graphs are topics for future research.

This chapter presented a Bond Graph approach to modeling human behaviors. This approach is a comprehensive alternative to the fluid analogy models that have ushered in a model-based approach to operationalizing the well-known human behavior theories. The ability to carry out causality analysis in the Bond Graph 
models serves to ensure that the energy transfers are consistent and the modelers intentions are accurately captured. It was demonstrated that the models help in designing controllers that can guide the selection of wellness interventions. In the future, hybrid bond graph models can be developed to better represent and reason about the interactions between the individual and their environment. 
CHAPTER IV

BOND GRAPH MODELS FOR THE CLIENT-THERAPIST INTERACTIONS

This Chapter presents Bond Graph models that describe the interaction between the Client and the Therapist. The main objective is to understand how to stimulate positive behaviors in individuals that lead to better management of their own wellness. Thus the need to describe the interaction during the therapy session or motivational interviews arises. This Chapter presents Bond Graph models for the client and therapist interaction based on the theory of planned behavior and the social cognitive theory in addition to the basic model. It is shown that, the proposed models of interaction offer many control options to analyze and maintain a healthy Client-Therapist relation.

As illustrated in the previous Chapter, Bond Graph approach has been applied for existing, well-known behavioral theories in order to construct dynamic models [4]. In this Chapter, it is first shown that the dynamics of Client-Therapist interaction can be derived from a Bond Graph model. The Bond Graph model is itself derived from a fluid analogy model for the Client and the Therapist in order to simplify the presentation. The value of causality analysis in this approach is demonstrated. In addition, it is shown that, this approach also leads to a similar 
conclusion about the stable states of the system as reported in [16]. The model for the Client-Therapist interaction has been extended based on the theory of planned behavior, the social cognitive theory and the simplified social cognitive theory. Bond graph models were obtained and the dynamic equations were derived. Further, state space representation was used to describe the dynamics in each case, and the models were analyzed to determine the gain margins of the different inputs.

\subsection{The Basic Client-Therapist Relation Model}

Human behavior is viewed as sequence of complex energy transfers across multiple domains. The interest is to model the behavior in order to improve decisionsupport $[22,70]$ and, hence, the aim is to develop actionable models for human behavior. In this section, a basic model for the interaction between a Client and a Therapist that is inspired by the work in [16] is presented.

A fluid analogy representation [4] for the interaction between a client and a therapist is demonstrated in Figure 4.1. There are two tanks for storing fluid — the client is represented by the storage tank on the right and the other tank represents the therapist. Following the ideas in [16], the level of fluid in each of these storage tanks corresponds to the valence, or affect, of the client $\left(h_{2}\right)$ and the therapist $\left(h_{1}\right)$. The valence of the therapist is a function of his or her training $\left(S_{1}\right)$ and is regulated by the valve $N_{1}$. It is assumed that a better trained therapist, i.e., more flow in $N_{1}$, would have higher valence. The valence of the client is affected by the environmental conditions $\left(S_{2}\right)$ and regulated by $N_{2}$. Through the therapy, the valence of the client 
and the therapist are changed because of the flows through the valve that is labeled Therapy $(R)$.

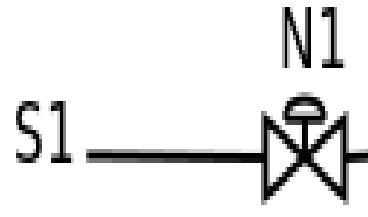

Figure 4.1: Fluid analogy representation for the interaction between a Therapist and a Client.

To construct a Bond Graph for the Client-Therapist interaction, the physical components of the system illustrated in in Figure 4.1 were identified and mapped to Bond Graph elements. The corresponding Bond Graph elements are shown in Table 4.1. 
Table 4.1: Physical components of the system and corresponding bond graph elements

\begin{tabular}{|c|c|c|}
\hline Physical Element & Type & Value \\
\hline Training source & Sf & $S_{1}$ \\
\hline Environment source & Sf & $S_{2}$ \\
\hline Training valve & $\mathrm{TF}$ & $N_{1}$ \\
\hline Environment valve & $\mathrm{TF}$ & $N_{2}$ \\
\hline Therapist valence & $\mathrm{C}$ & $L_{1}$ \\
\hline Client valence & $\mathrm{C}$ & $L_{2}$ \\
\hline Therapy & $\mathrm{R}$ & $R$ \\
\hline
\end{tabular}

As illustrated in Chapter 2, every distinct effort point in the system was represented by a 0 -Junction and the Bond Graph elements were connected between these effort points using 1-Junctions and half arrows. The Bond Graph was simplified and the resulting model after causality analysis is shown in Figure 4.2. 


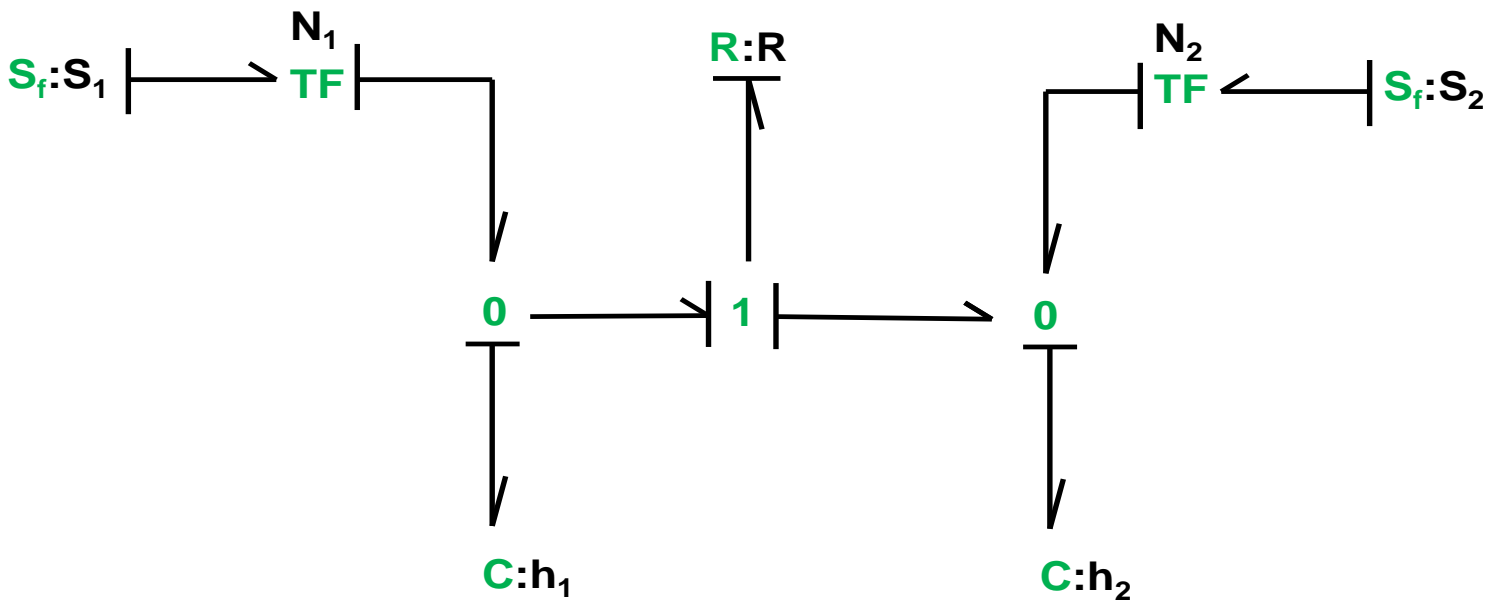

Figure 4.2: Bond graph representation for the Client-Therapist model

The dynamic behavior of the system that was obtained using the procedure in $[31]$ is

$$
\begin{aligned}
\frac{d h_{1}}{d t} & =\frac{S_{1} N_{1}}{\rho g C_{1}}-\frac{1}{R}\left(h_{1}(t)-h_{2}(t)\right) \\
\frac{d h_{2}}{d t} & =\frac{S_{2} N_{2}}{\rho g C_{2}}+\frac{1}{R}\left(h_{1}(t)-h_{2}(t)\right)
\end{aligned}
$$

Equations (4.1) show how the valence of the client and therapist change over time. Since these are the only state variables in the system, these two equations 
collectively describe the behavior of the interaction system. These equations are identical to the behavior presented in [16].

\subsubsection{Regulating the Dynamic Behavior of the Client-Therapist Relation}

The Bond Graph model allowed us to systematically obtain a set of dynamic equations for a subjective system. Now this dynamic model in Equation 4.1 can be used to regulate how the Client-Therapist relation evolves. To illustrate this idea, the model was used to regulate the client valence through therapy using three different control schemes, the Client response under each scheme was recorded. To maintain the Client valence, it is assumed that, there is an optimal level, $L_{c}$, that should be maintained. A well trained therapist should be able to control the ("flow of") therapy (offered) to the client and the ("flow of") feedback from the client. To model this interaction, controllers were designed to regulate the valve $R$. The integrated system was represented as a multiple inputs and multiple outputs (MIMO) system. First, the transfer function of the system should be obtained from the dynamic equations that were derived using the Bond Graph. From Equation 4.1, the Client's valance can be expressed in terms of the therapist training $\left(S_{1}, N_{1}\right)$, the environment inputs $\left(S_{2}, N_{2}\right)$ and the therapy regulation valve $(R)$ as

$$
h_{2}(s)=\left[\begin{array}{c}
\frac{1}{s^{2}+\left(R^{2}+2 R\right) s+\left(R^{3}+R^{2}\right)} \\
\frac{R s}{s^{2}+\left(R^{2}+2 R\right) s+\left(R^{3}+R^{2}\right)}
\end{array}\right] \times\left[\begin{array}{ll}
S_{1} N_{1} & S_{2} N_{2}
\end{array}\right]
$$


The MIMO transfer function was obtained from the Equation 4.2 as

$$
H(s)=\left[\begin{array}{c}
\frac{1}{s^{2}+\left(R^{2}+2 R\right) s+\left(R^{3}+R^{2}\right)} \\
\frac{R s}{s^{2}+\left(R^{2}+2 R\right) s+\left(R^{3}+R^{2}\right) .}
\end{array}\right]
$$

From this point, different controllers can be explored in order to regulate behaviors in this interaction.

Maintaining Client Valence using Proportional Controller Under proportional Control, the controller transfer function is only one term of proportional ratio $\left(K_{p}\right)$ [71]. Only the first entry of the transfer function is considered, since the training and the therapy valves can be controlled in order to regulate the valance of the client. Environment context on the other hand is not easy to be manipulated. A unit step value is selected as input for both sources and we assumed that the transformers have unit ratios. The therapy valve was assumed to be $50 \%$ opened. The transfer function can be written as

$$
H_{1}(s)=\frac{1}{6.25 s^{2}+7.5 s+1}
$$

After integrating the proportional controller $\left(K_{p}\right)$ to the system, the response of the system to a unit set point was depicted in Figure 4.3. 


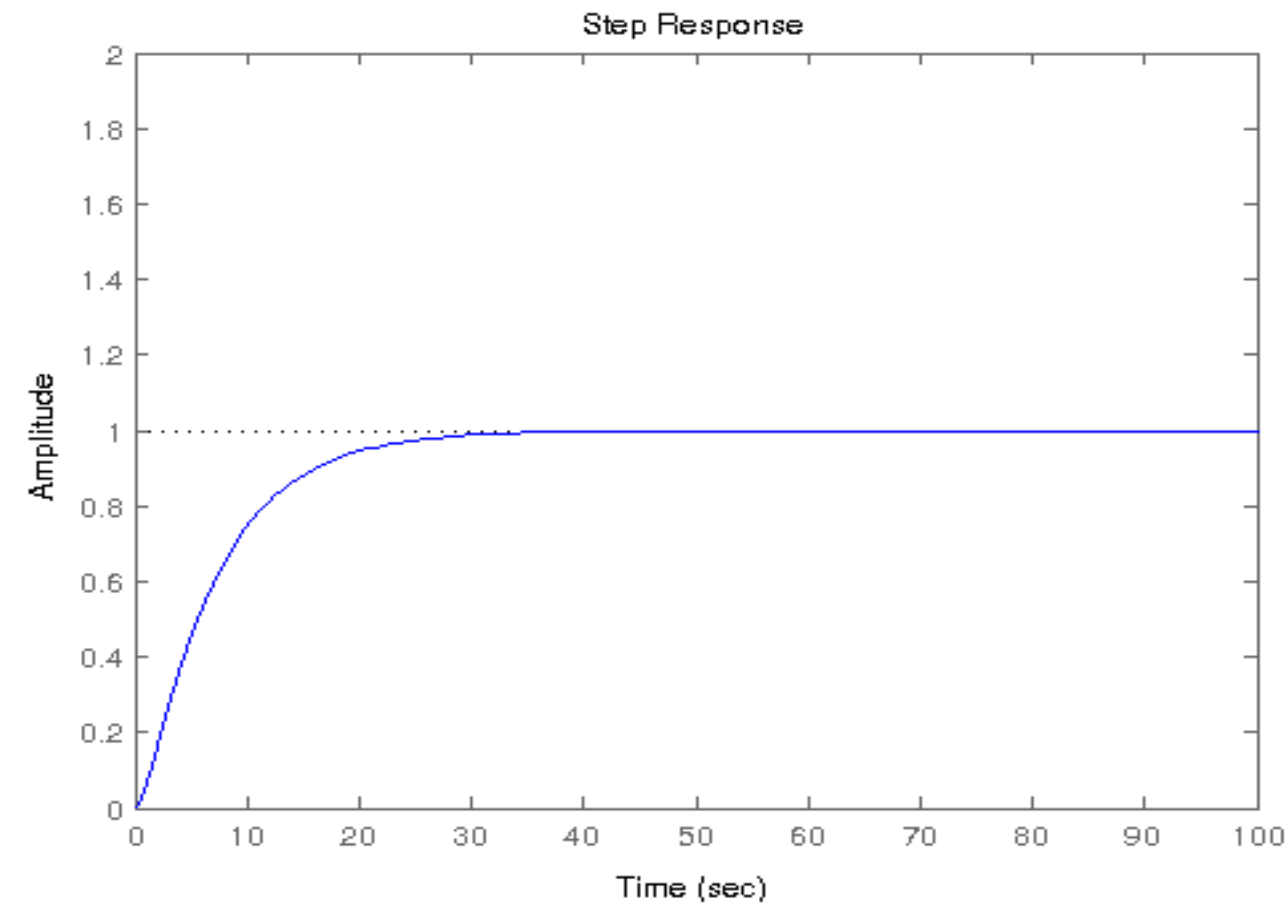

Figure 4.3: The step response of the client valence under the proportional controller

Maintaining Client Valence using Proportional and Integral Controller

Because of the delay in the response of the proportional Control, an integral part was added to the controller. The new transfer function of the controller now has two terms $\left(K_{p}+\frac{K_{i}}{s}\right)$ [71]. Again the transfer function between the Client's valance level and the training and therapy valves is considered, using the same values as in the first case yields the transfer function as

$$
H_{1}(s)=\frac{10 s+1}{6.25 s^{2}+7.5 s+10 s+1}
$$


After connecting the proportional integral controller $\left(K_{p}+\frac{K_{i}}{s}\right)$ to the system, the response of the system to a unit set point was depicted in Figure 4.4.

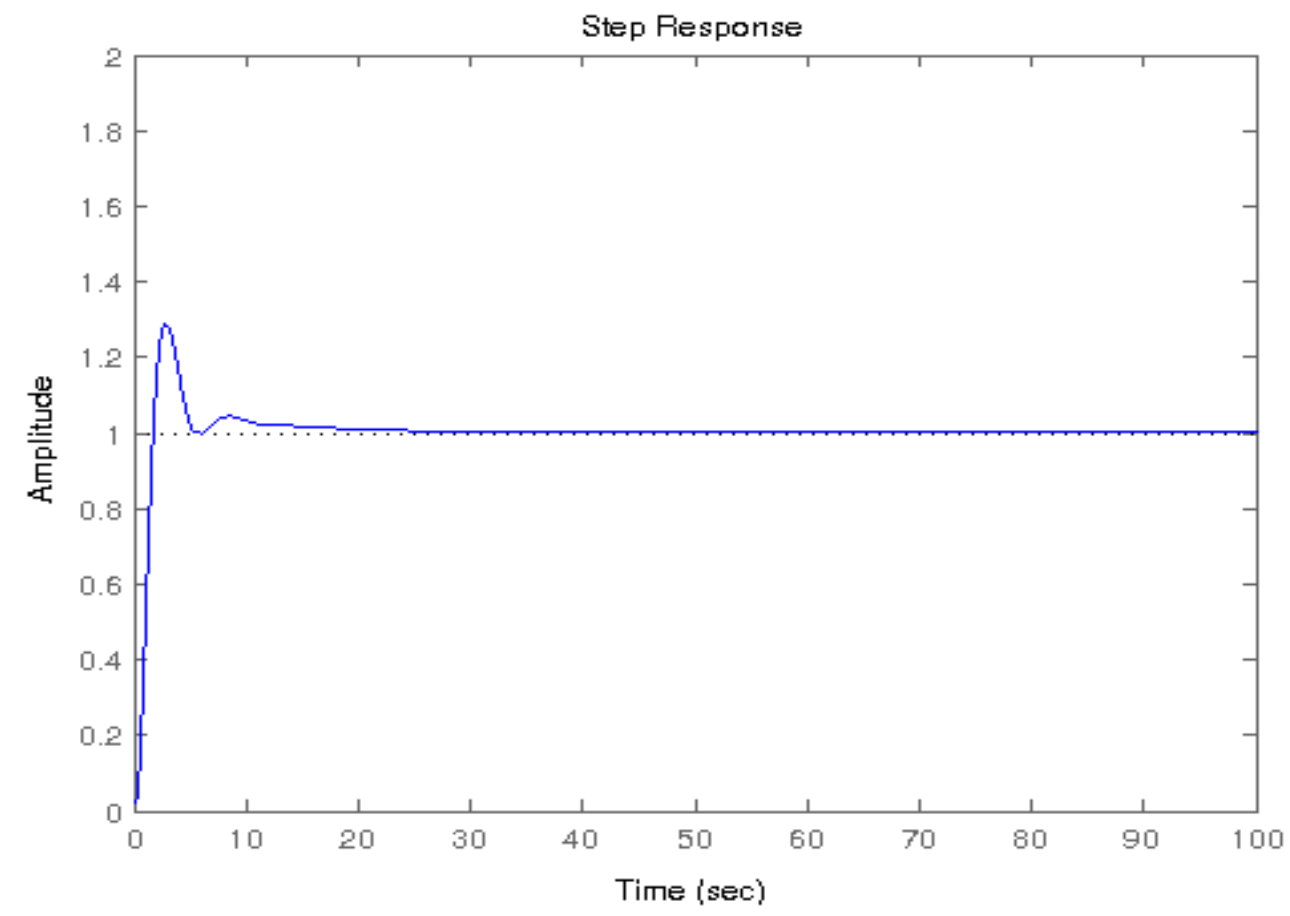

Figure 4.4: The step response of the client valence under the proportional and integral controller

Maintaining Client Valence using Proportional, Integral and Derivative Controller The addition of the integral part to the controller makes the response faster, but it causes an overshooting in the system response. A derivative part was added to the controller to limit this overshooting. The transfer function now becomes $\left(K_{p}+\frac{K_{i}}{s}+K_{d} \times s\right)[71]$. Again, considering just the relation between the client's valence, and the training and therapy valves, using the same values as in the first case, the equivalent transfer function is 


$$
H_{1}(s)=\frac{10 s^{2}+10 s+1}{6.25 s^{2}+17.5 s+10 s+1}
$$

With this proportional, integral and derivative controller, the response of the system to a unit set point is shown in Figure 4.5.

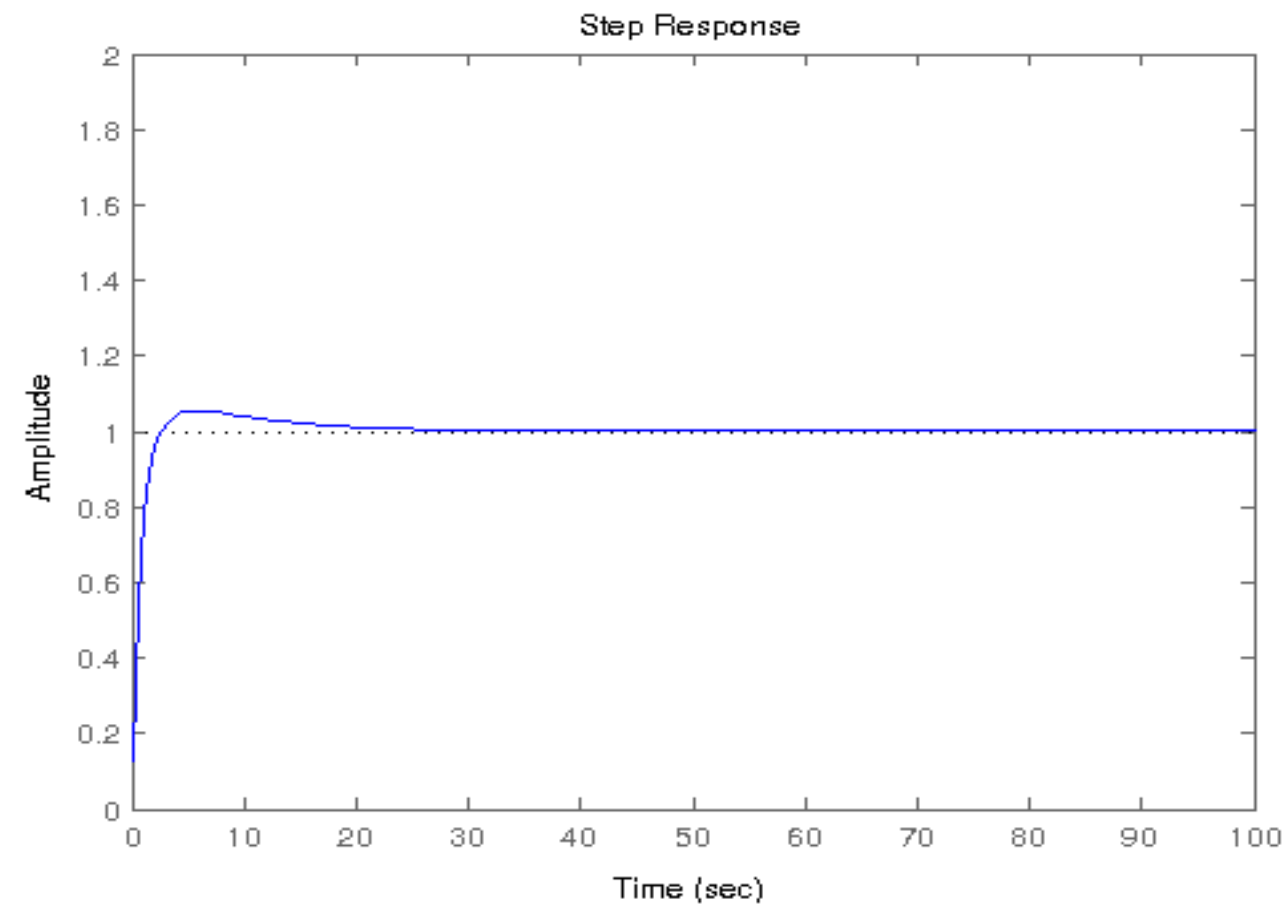

Figure 4.5: The step response of the client valence under the proportional, integral and derivative controller

It can be seen from these results that, the controllers that were used to regulate the interactions offered a variety of choices. Among these controllers, the PID controller provides minimum delay and minimum overshoot; it is however difficult to tune. 


\subsubsection{Examining the Client-Therapist Relationship}

The model shown in Equation (4.1) was also used to analyze the stability of the system. The critical points of this system were identified and the system trajectories were plotted starting from different initial conditions, i.e., different initial states of both the Client and the Therapist. For example, if the therapist has initially positive valence, how is that going to affect the initially negative, neutral or positive client. Using the parameters from [16], the phase portraits were plotted as shown in Figure 4.6.

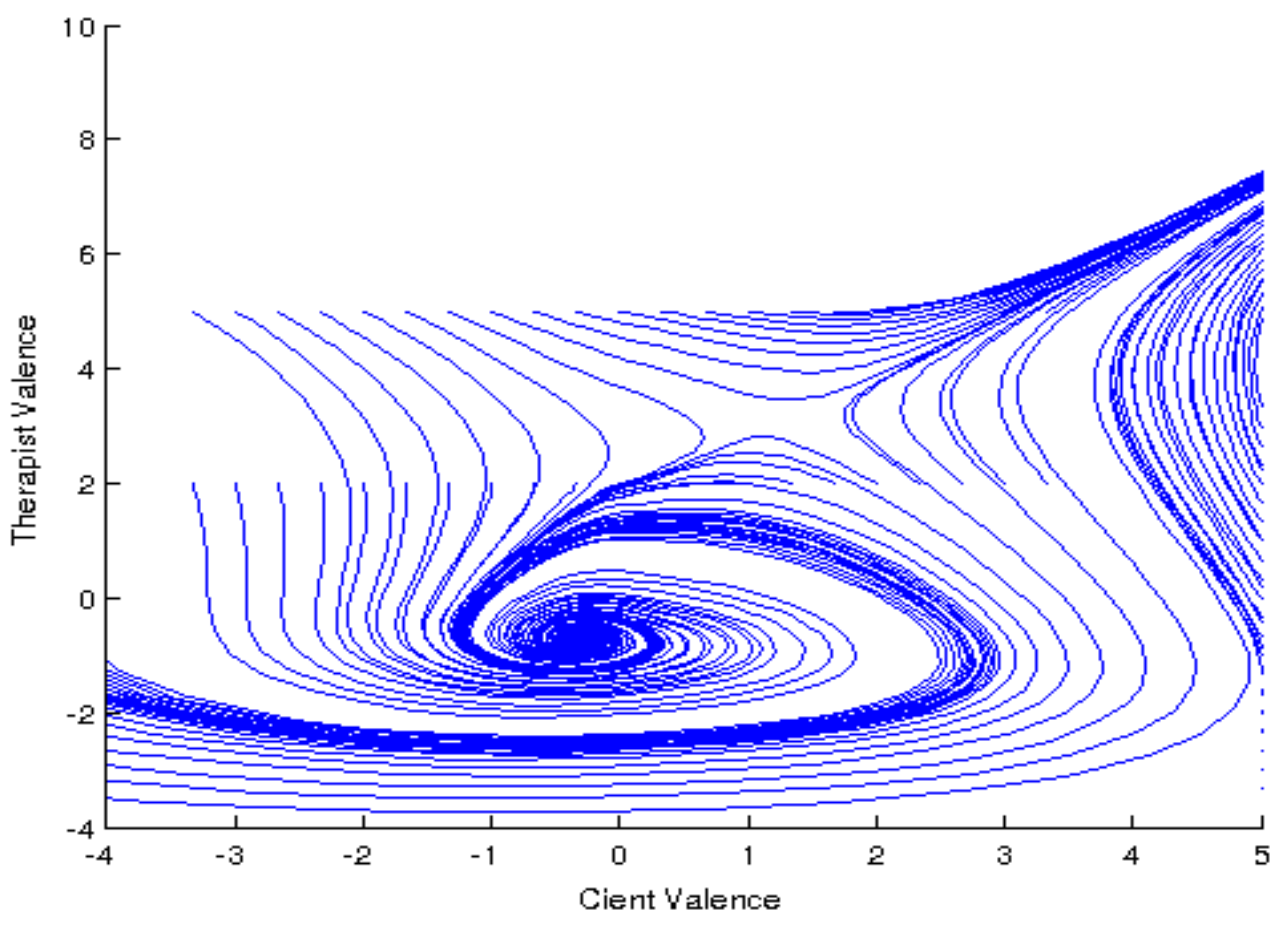

Figure 4.6: The phase portraits for the psychotherapy relation 
Notice from Figure 4.6 that, there are two critical points - the first is the stable point, Attractor, which is the point reached as a conclusion of a successful therapy program. The other point is the saddle point that represents a failed therapy. The figure shows that, when the therapist has a positive valence, the therapy sessions can lead a client with a negative or positive valence to the attractor. On the other hand a when the therapist has a negative valence, the session will conclude in the saddle point. These two stable points are similar to the ones reported in [16].

It can be seen from these results that the Bond Graph approach is useful for obtaining dynamical system model of the Client-Therapist interaction as illustrated in $[16]$.

The next step is to extend the client side of the Bond Graph model to capture the parameters and environmental triggers in the various constructs for human behavior in the Bond Graph models.

\subsection{Client-Therapist Relation Using The Theory of Planned Behavior}

A more detailed model for the client offers may more flexibility and control options to achieve target behaviors. The constructs of the theory for human behavior also allow us to understand the observed behaviors at a higher resolution.

For instance, in a system with one controlled variable there is only one transfer function. If the characteristic equation of such system fails the stability test, then no control is possible. Even when the system is stable, only a simple controller can be added to regulate the valance of the client as described earlier. 
Human behavior is, in fact, much more complicated than the model presented in 4.2. To cope with complexity, model for the client based on the well-known Theory of Planned Behavior (TPB) [10, 4] was designed.

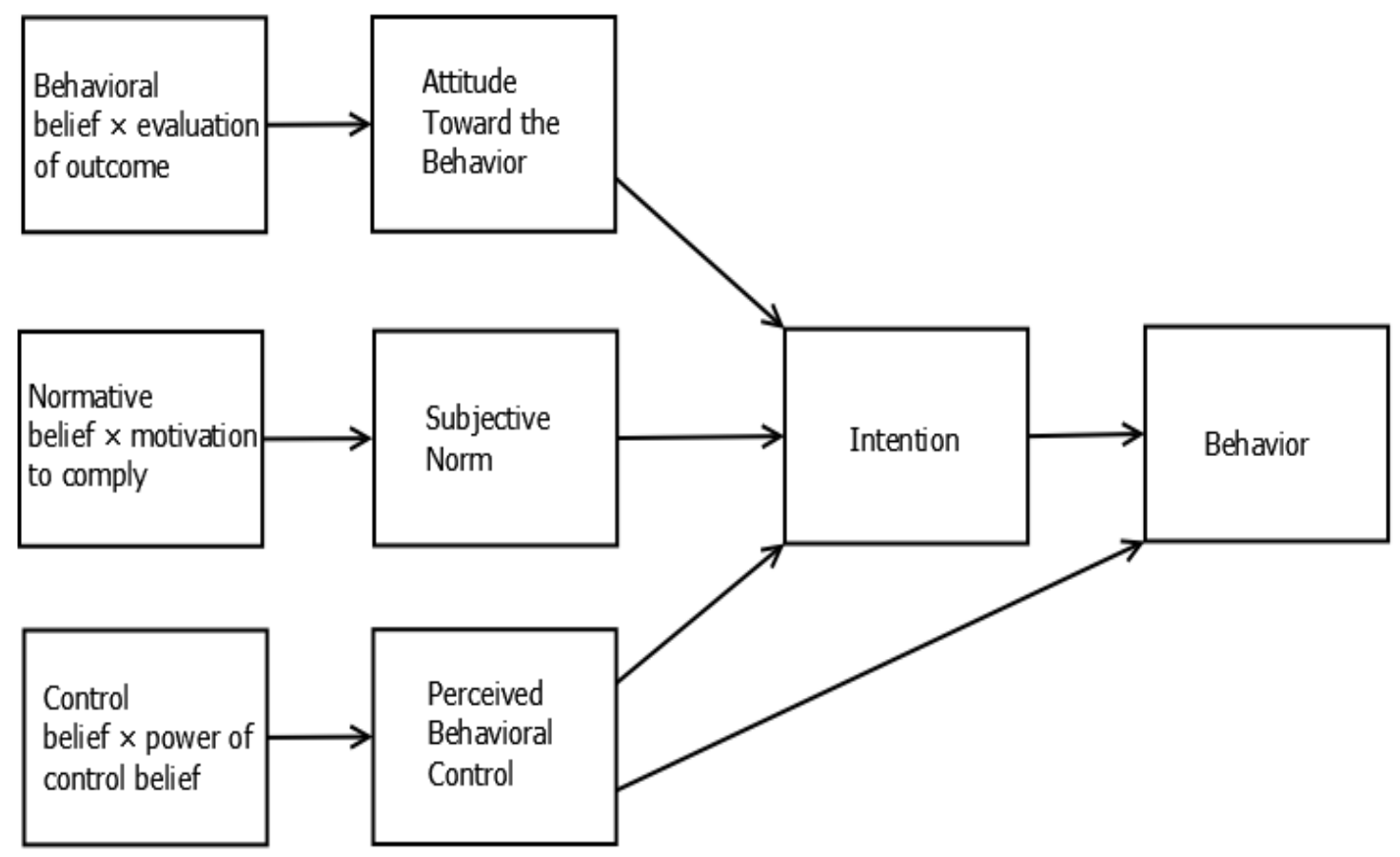

Figure 4.7: Block diagram of the Theory of Planned Behavior.

As mentioned in the previous Chapter, the major constructs of TPB, shown in Figure 4.7, are Attitude $\left(\eta_{1}\right)$, Social Norms $\left(\eta_{2}\right)$, Perceived Behavioral Control $\left(\eta_{3}\right)(\mathrm{PBC})$, Intention $\left(\eta_{4}\right)$ and Behavior $\left(\eta_{5}\right)$. These constructs are represented as storage elements in the Bond Graph model shown in Figure 4.8; these elements are the five state variables in the model. The input sources are Behavioral Belief $\left(\xi_{1}\right)$, Normative Belief $\left(\xi_{2}\right)$ and Control Belief $\left(\xi_{1}\right)$; these are modeled as sources of flow 
in the Bond Graph model. The uncertainties associated with the state variables are modeled as disturbances and all these are also modeled as sources of flow $\left(\varsigma_{1}, \varsigma_{2}, \cdots\right.$, $\left.\varsigma_{5}\right)$ in the bond graph model.

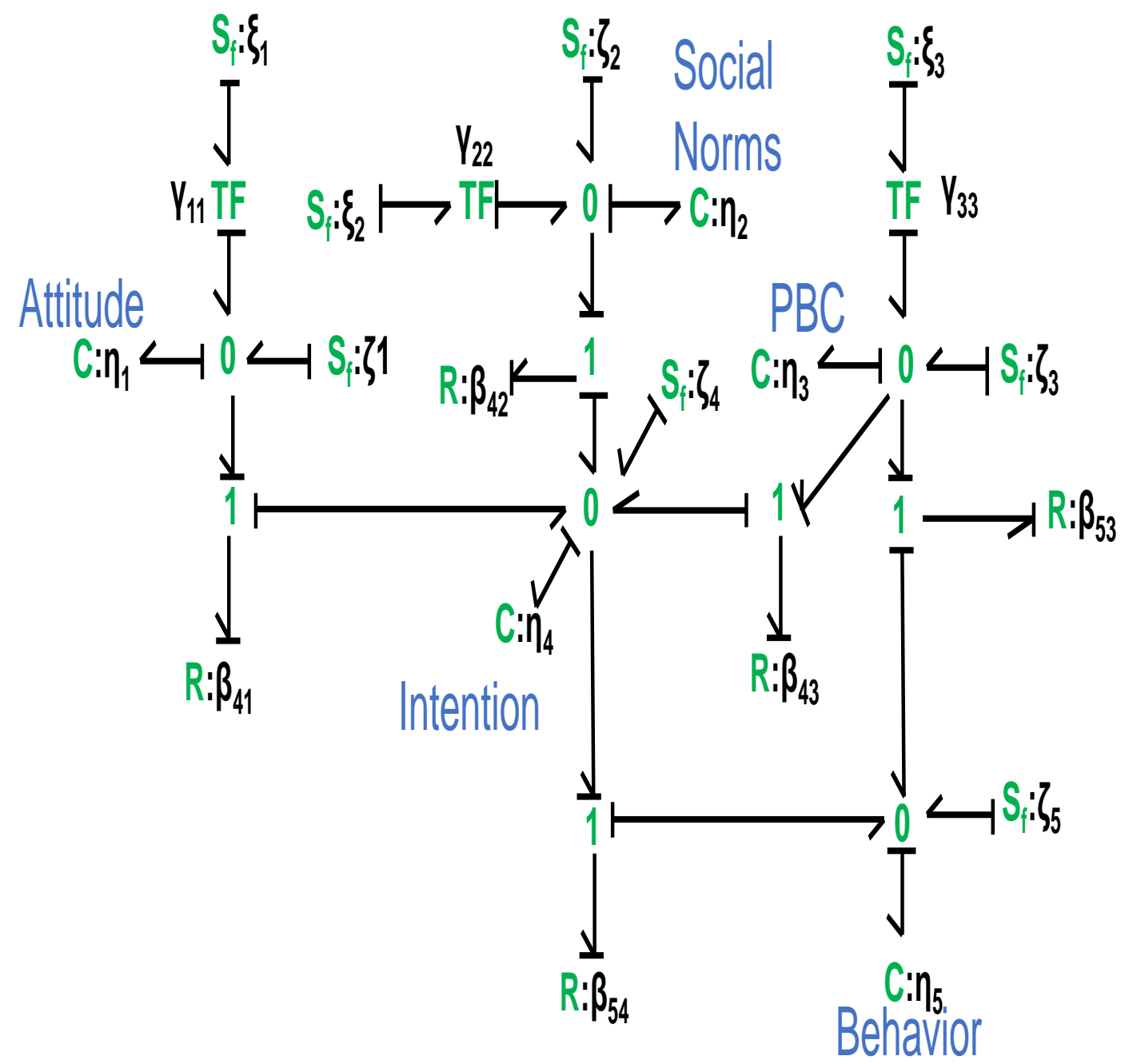

Figure 4.8: Bond graph model based on the Theory of Planned Behavior [4]. 
The dynamic equations obtained from the Bond Graph are:

$$
\frac{d \eta_{1}}{d t}=\gamma_{11} \xi_{1}-\frac{1}{\beta_{41}}\left(\eta_{1}-\eta_{4}\right)+\varsigma_{1}
$$

$$
\frac{d \eta_{2}}{d t}=\gamma_{22} \xi_{2}-\frac{1}{\beta_{42}}\left(\eta_{2}-\eta_{4}\right)+\varsigma_{2}
$$

$$
\frac{d \eta_{3}}{d t}=\gamma_{33} \xi_{3}-\frac{1}{\beta_{43}}\left(\eta_{3}-\eta_{4}\right)-\frac{1}{\beta_{53}}\left(\eta_{3}-\eta_{5}\right)+\varsigma_{3}
$$

$$
\frac{d \eta_{4}}{d t}=\frac{1}{\beta_{41}}\left(\eta_{1}-\eta_{4}\right)+\frac{1}{\beta_{42}}\left(\eta_{2}-\eta_{4}\right)+\frac{1}{\beta_{43}}\left(\eta_{3}-\eta_{4}\right)-\frac{1}{\beta_{54}}\left(\eta_{4}-\eta_{5}\right)+\varsigma_{4}
$$

$$
\frac{d \eta_{5}}{d t}=\frac{1}{\beta_{53}}\left(\eta_{3}-\eta_{5}\right)+\frac{1}{\beta_{54}}\left(\eta_{4}-\eta_{5}\right)+\varsigma_{5}
$$

Now if the Client's valence tank in Figure 4.1 is replaced with the TPB model shown in Figure 4.7, a more detailed model that describes the interaction between a Therapist and Client is obtained as shown in Figure 4.9. 


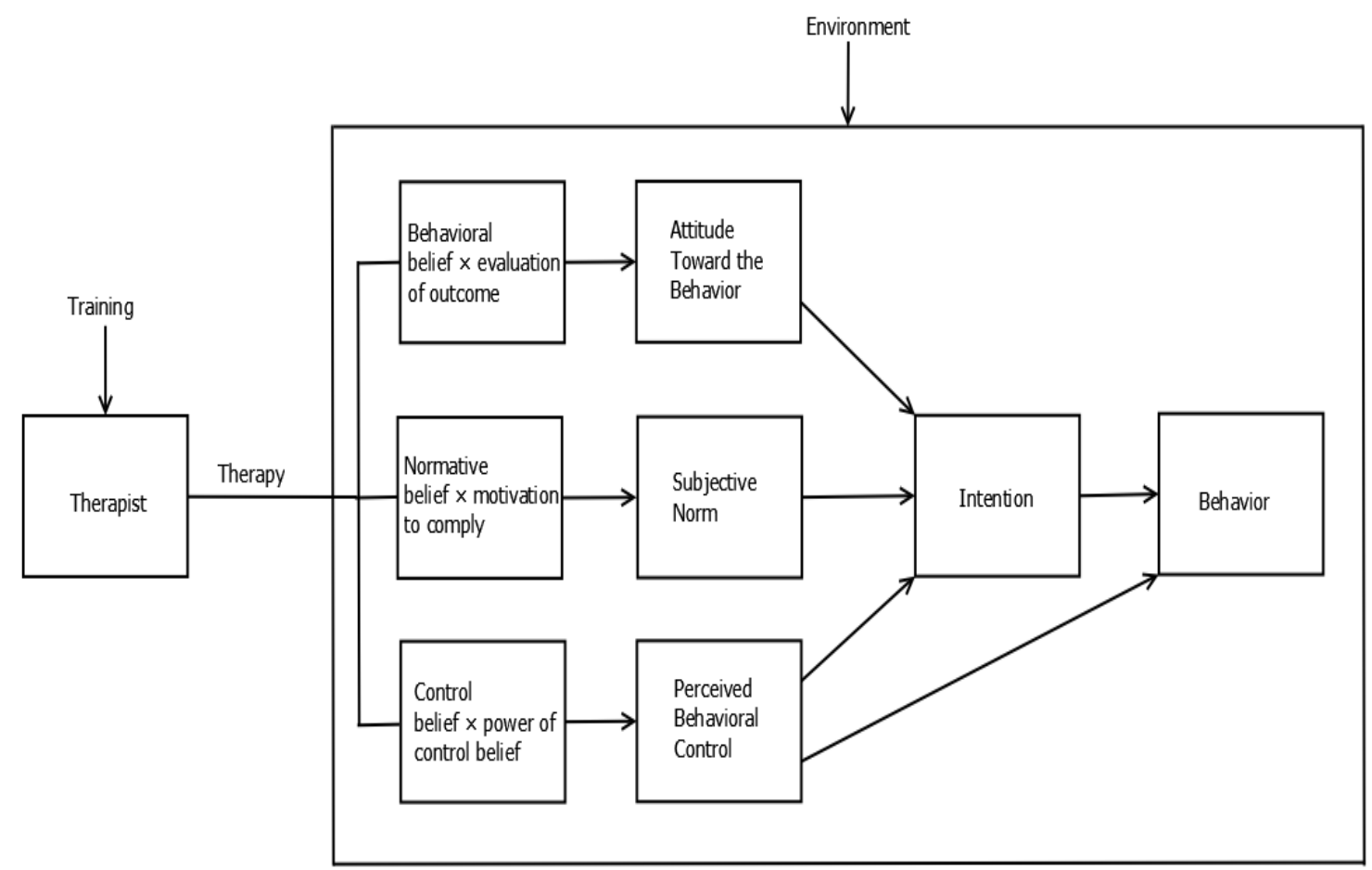

Figure 4.9: Client-Therapist block diagram based on the Theory of Planned Behavior.

The Bond Graph of this new Client-Therapist model can also be obtained by allowing the therapist to regulate all the inputs of the TPB model as illustrated in Figure 4.10. 


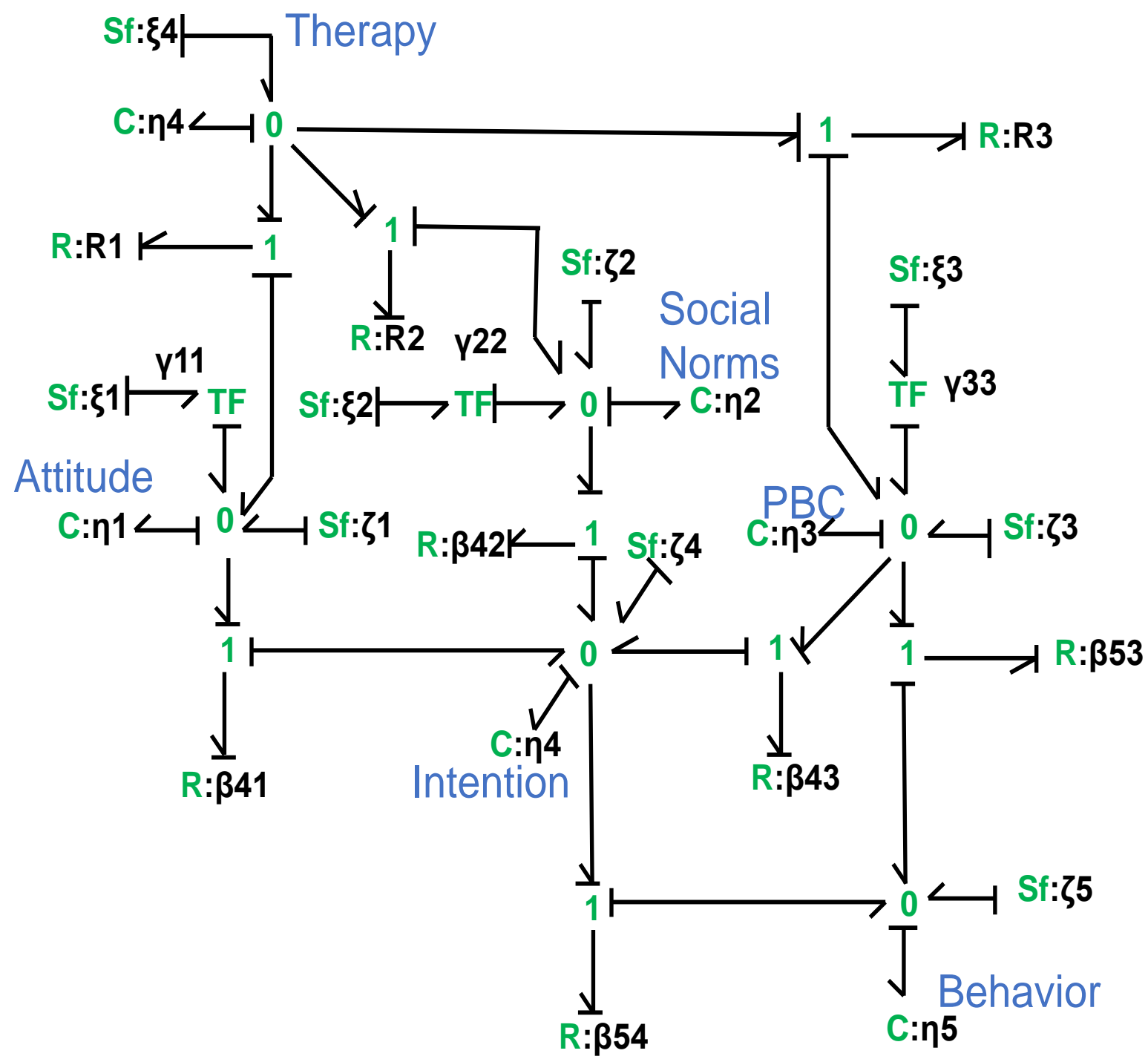

Figure 4.10: Bond graph model for the Client-Therapist relation based on the Theory of Planned Behavior.

This new dynamic model for the MIMO Client-Therapist interaction offers more control options. It is necessary, however, to assess the stability of the system using tools, such as the Root Locus and Bode Plots [71]. The Root Locus method allows one to determine whether the system is stable, critically stable or not stable. 
Controllers can only be designed when the system is stable or critically stable. Bode Plots allow us to determine the gain margin and phase margin for each input. In this case, it is important to understand these margins for the three inputs in the TPB Bond Graph model. The relative levels of the margins can be used by the therapist to identify the most influential therapy that can be offered to the client. The specific values of the margins can be used to determine the intensity of the intervention (therapy), perhaps guided by an online controller that works besides the therapist.

\subsubsection{State-Space Representation}

The dynamic behavior of the interaction that is derived from the Bond Graph model allows us to view the interaction as a MIMO system that receive inputs and generate outputs. The inputs to this system are behavioral beliefs $\xi_{1}$, normative beliefs $\xi_{2}$ and control beliefs $\xi_{3}$. The states are the levels of the storage elements - Attitude

$\left(\eta_{1}\right)$, Social Norms $\left(\eta_{2}\right)$, Perceived Behavioral Control $\left(\eta_{3}\right)(\mathrm{PBC})$, Intention $\left(\eta_{4}\right)$ and Behavior $\left(\eta_{5}\right)$, while the Behavior $\left(\eta_{5}\right)$ is considered as the only output.

The state vector of the system can be represented as

$$
X=\left[\begin{array}{lllll}
\eta_{1} & \eta_{2} & \eta_{3} & \eta_{4} & \eta_{5}
\end{array}\right] \cdot
$$

The state vector can change over time as

$$
\frac{d X}{d t}=\left[\begin{array}{lllll}
\frac{d \eta_{1}}{d t} & \frac{d \eta_{2}}{d t} & \frac{d \eta_{3}}{d t} & \frac{d \eta_{4}}{d t} & \frac{d \eta_{5}}{d t}
\end{array}\right] \cdot^{T}
$$


The input vector to the system is

$$
U=\left[\begin{array}{llllllll}
\xi_{1} & \xi_{2} & \xi_{3} & \zeta_{1} & \zeta_{2} & \zeta_{3} & \zeta_{4} & \zeta_{5}
\end{array}\right]^{T} \cdot
$$

In order to formulate the dynamics of the system in the state-space representation [72], four matrices, $A, B, C$, and $D$ are defined as follows:

$$
\begin{aligned}
& A=\left[\begin{array}{ccccc}
-\frac{1}{\beta_{41}} & 0 & 0 & \frac{1}{\beta_{41}} & 0 \\
0 & -\frac{1}{\beta_{42}} & 0 & \frac{1}{\beta_{42}} & 0 \\
0 & 0 & -\frac{1}{\beta_{43}}-\frac{1}{\beta_{53}} & \frac{1}{\beta_{43}} & \frac{1}{\beta_{53}} \\
\frac{1}{\beta_{41}} & \frac{1}{\beta_{42}} & \frac{1}{\beta_{43}} & -\frac{1}{\beta_{41}}-\frac{1}{\beta_{42}}-\frac{1}{\beta_{43}}-\frac{1}{\beta_{54}} & \frac{1}{\beta_{54}} \\
0 & 0 & \frac{1}{\beta_{53}} & \frac{1}{\beta_{54}} & -\frac{1}{\beta_{53}}-\frac{1}{\beta_{54}}
\end{array}\right] \\
& B=\left[\begin{array}{cccccccc}
\gamma_{11} & 0 & 0 & 1 & 0 & 0 & 0 & 0 \\
0 & \gamma_{22} & 0 & 0 & 1 & 0 & 0 & 0 \\
0 & 0 & \gamma_{33} & 0 & 0 & 1 & 0 & 0 \\
0 & 0 & 0 & 0 & 0 & 0 & 1 & 0 \\
0 & 0 & 0 & 0 & 0 & 0 & 0 & 1
\end{array}\right] \\
& C=\left[\begin{array}{lllll}
0 & 0 & 0 & 0 & 1
\end{array}\right] . \\
& D=\left[\begin{array}{llllllll}
0 & 0 & 0 & 0 & 0 & 0 & 0 & 0
\end{array}\right]
\end{aligned}
$$


The matrix $A$ is the state matrix that relates the state change vector $\left(\frac{d X}{d t}\right)$ to the state $X$. The matrix $B$ is the input matrix that relates the state-change vector to the input vector $(U)$. The output matrix, $C$, relates $X$ to the output vector, $Y$. Finally, the feed through matrix, $D$, relates $U$ to $Y$. In this system, there is no direct connection between any input to the behavior.

The dynamic behavior the system derived from the Bond Graph model in Figure 4.10 can now be represented in a state-space formulation as

$$
\begin{array}{r}
\frac{d X}{d t}=A \times X+B \times U \\
Y=C \times X+D \times U
\end{array}
$$

In order to evaluate the stability of the system, a transfer function for the interaction is needed. The transfer function of this MIMO system [72] is

$$
H(s)=C \times\left((s * I-A)^{-1}\right) \times B+D .
$$

\subsubsection{Stability Analysis}

Stability analysis is required before designing controllers. One method is to compute the poles of the characteristic equation of the system and plot them on the Complex Plane. Poles on the positive real part indicate that the system is unstable. The system is said to be critically stable if any of the poles are on the imaginary axis. The system is stable, otherwise [71]. 
The Root Locus method is used to compute the poles of the system and plot them as shown in Figure 4.11. The values for the parameters in the transfer function, i.e., Equation (4.20), were selected to represent the situation where an individual lacked the intention for exercise. The Behavioral beliefs input was set to 0.1, Normative belief of value of -0.5 , and Control belief of value of 0.7 , all the transfer ratios of the inputs were assumed to be 1 . The resistance values between storage elements were selected to be $50 \%$.

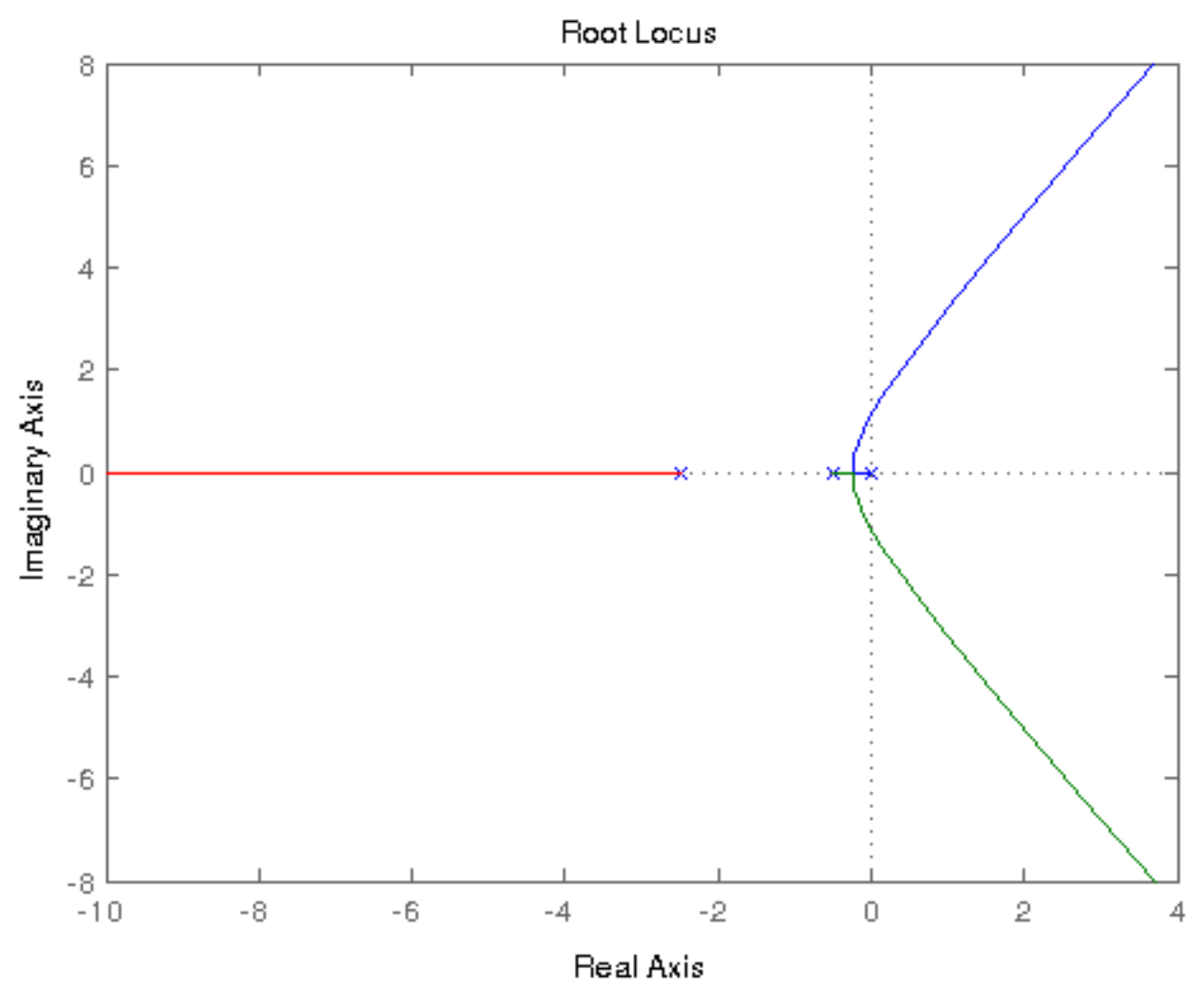

Figure 4.11: The position of the poles on the Complex Plane indicate that the system is critically stable. 
As seen from Figure 4.11, the system is critically stable. In such cases the controller must be designed carefully by calculating the gain and phase margins of stability [73]. For this reason, Bode Plots were used as discussed next.

\subsubsection{Stability Margins}

The key idea for computing stability margins is to plot the gain and the phase of the transfer function. The three inputs were selected as discussed above and the output was observed for the Behavior level. Figure 4.12 shows the gain and phase margins obtained. Here, the bottom set of figure shows the phase margin of input signal for each of the TPB inputs $-\xi_{1}, \xi_{2}$ and $\xi_{3}$. The top part of the figure shows the gain margins for the corresponding inputs. Since the phase plot of the third input, i.e., the Control belief does not cross the -180 phase, then the gain margin for this input is infinite, unlike the other two inputs which have limited gain margins. It is concluded from this analysis that, the Control belief input can be freely used to regulate the Behavioral input for the client. 


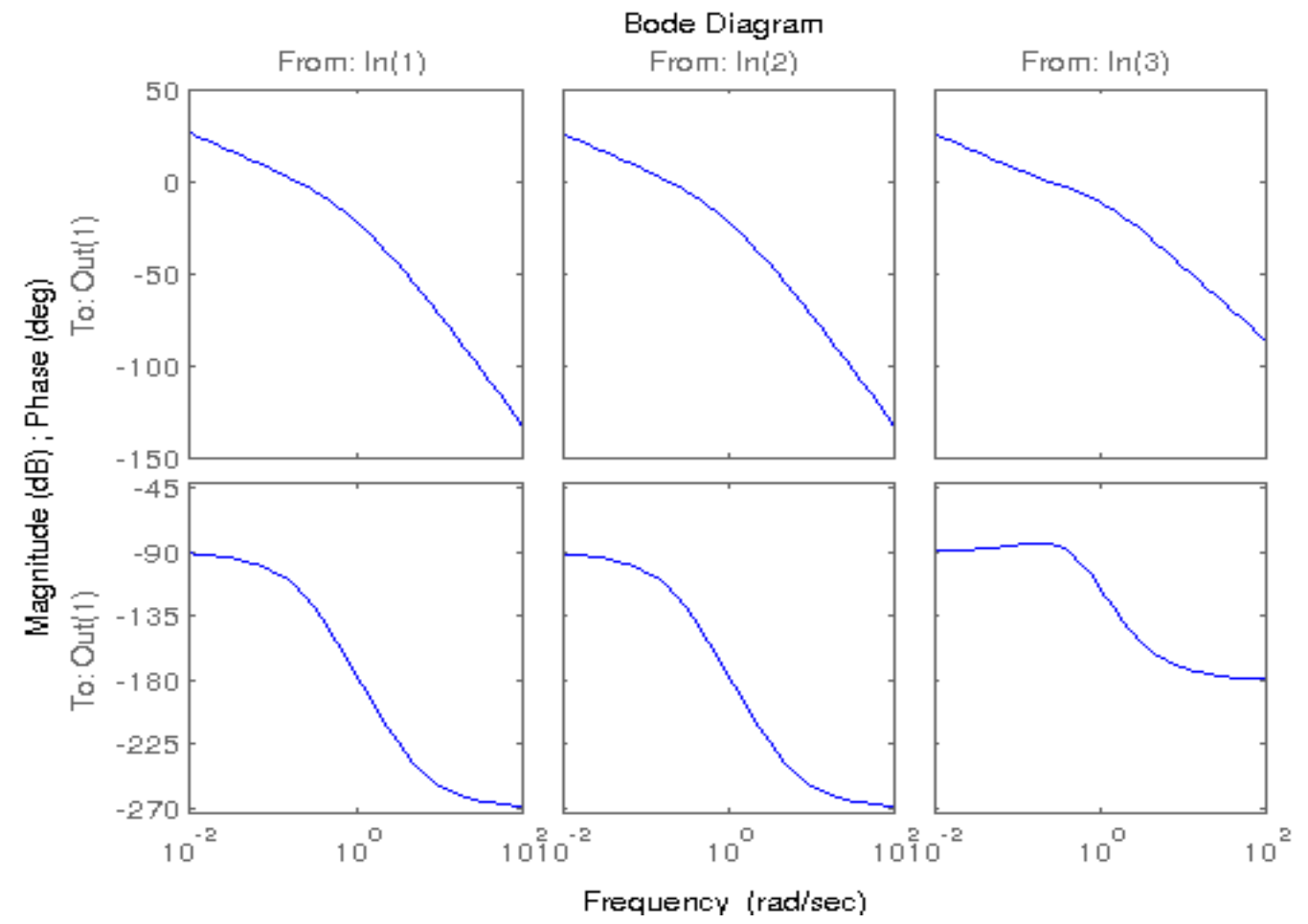

Figure 4.12: Gain and phase Bode plots for the Behavior output of the TPB.

To understand the value of such analysis, the system was evaluated using different values for the two resistor elements $\beta_{53}$ and $\beta_{54}$; these two resistors directly influence the value of the storage element $\eta_{5}$, which is the Behavior we wish to observe. Three different values were considered for each resister element, low resistance(10\%), medium resistance (50\%) and high resistance (90\%). For each of the nine possible combinations, Bode plots were generated to compute the gain and phase margins and identified the input variable with the highest margins. The results are presented in Figure 4.13. As can be seen, the Control Belief input is the best variable to regulate in the TPB model for the client in most cases. When the client 
has the ability to directly translate their intentions to behavior and the is strong enough to believe that they can carry out the suggested actions, then it is better for the therapist to regulate either the Behavior Belief input or the Normative Belief input.

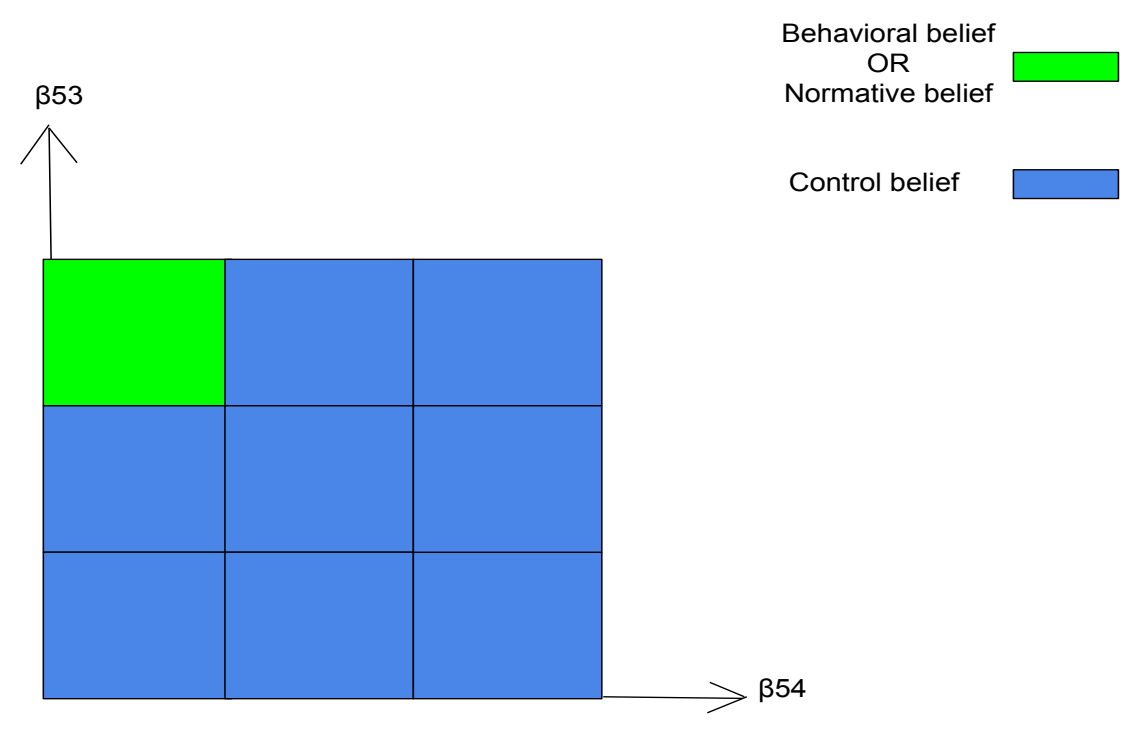

Figure 4.13: Plot shows the best input variable to be chosen as manipulated variable in each combination of resistances values in the Theory of Planned Behavior model. Notice that, the Control belief is recommended in eight out of the nine regions

\subsubsection{Regulating Behavior}

In order to maintain the behavior level for a specific individual at a certain level, a controller can be designed to adjust the flow. The controller can serve as a reference 
for the therapist, who can then select the level of intervention that may be useful for the client.

To illustrate these ideas, again the same values as in the previous sections were selected for all the parameters in the transfer function shown in Equation (4.20). The PID controller was connected to the system as shown in Figure 4.14. The controller was tuned based on the parameter values selected. The Control belief $\left(\xi_{3}\right)$ input was selected as controlled variable because of its large gain margin. The response obtained using this PID Controller for an individual with initial behavior level 0 and desired behavior at 11, is shown in Figure 4.15. Notice that the response is close to the desired behavior level. In contrast, the same controller was used to adjust the Behavior by selecting the Behavior belief $\left(\xi_{1}\right)$ input as controlled variable. The result is shown in Figure 4.16. Notice that, the response in this case is not as close as the first case to the desired Behavior level. 


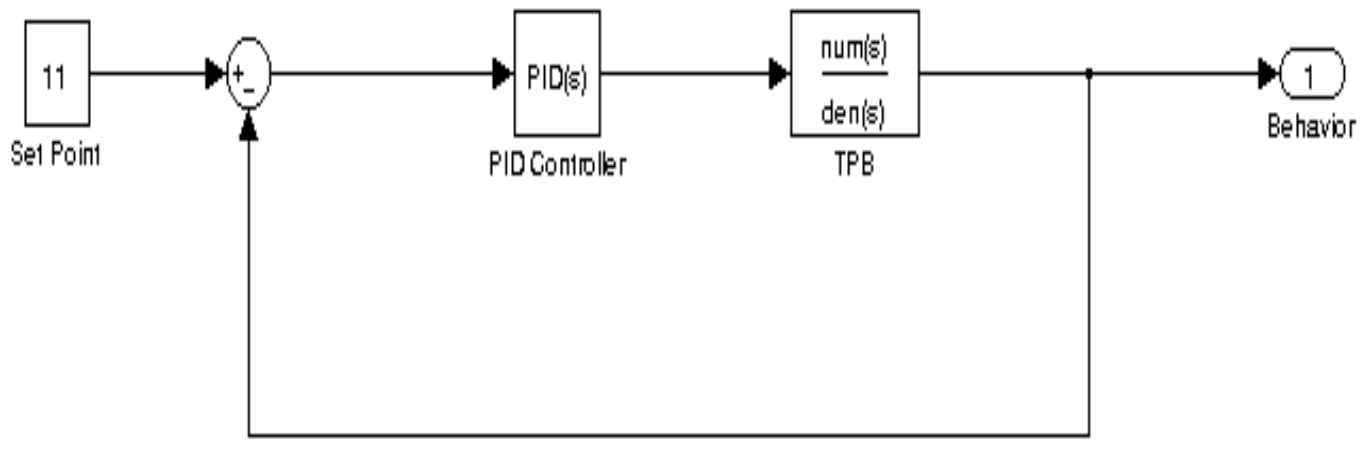

Figure 4.14: Control block diagram of the TPB model integrated with a PID controller. 


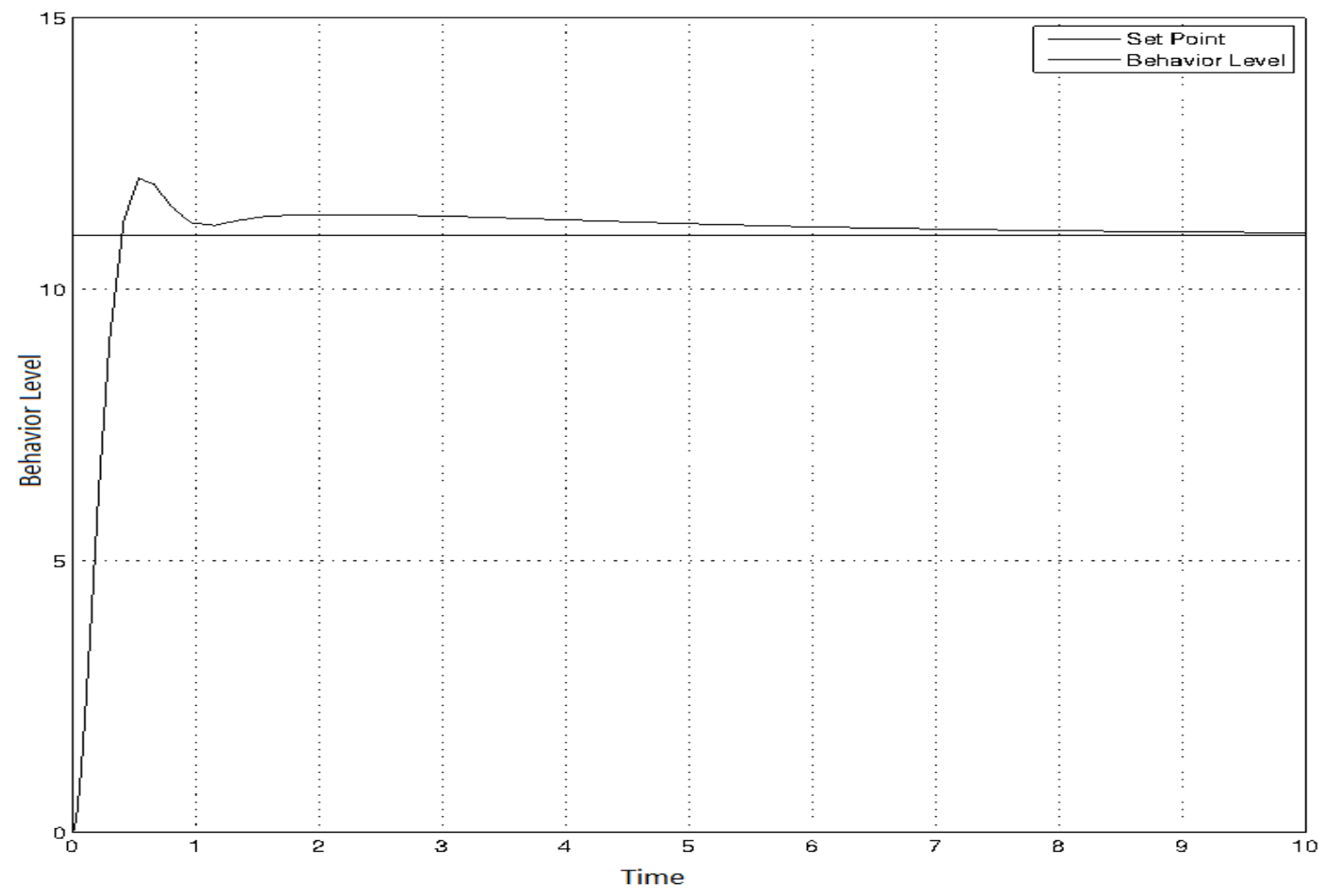

Figure 4.15: The response of the individual's Behavioral level for a step change of 11 when Control belief $\left(\xi_{3}\right)$ is used as controlled variable. 


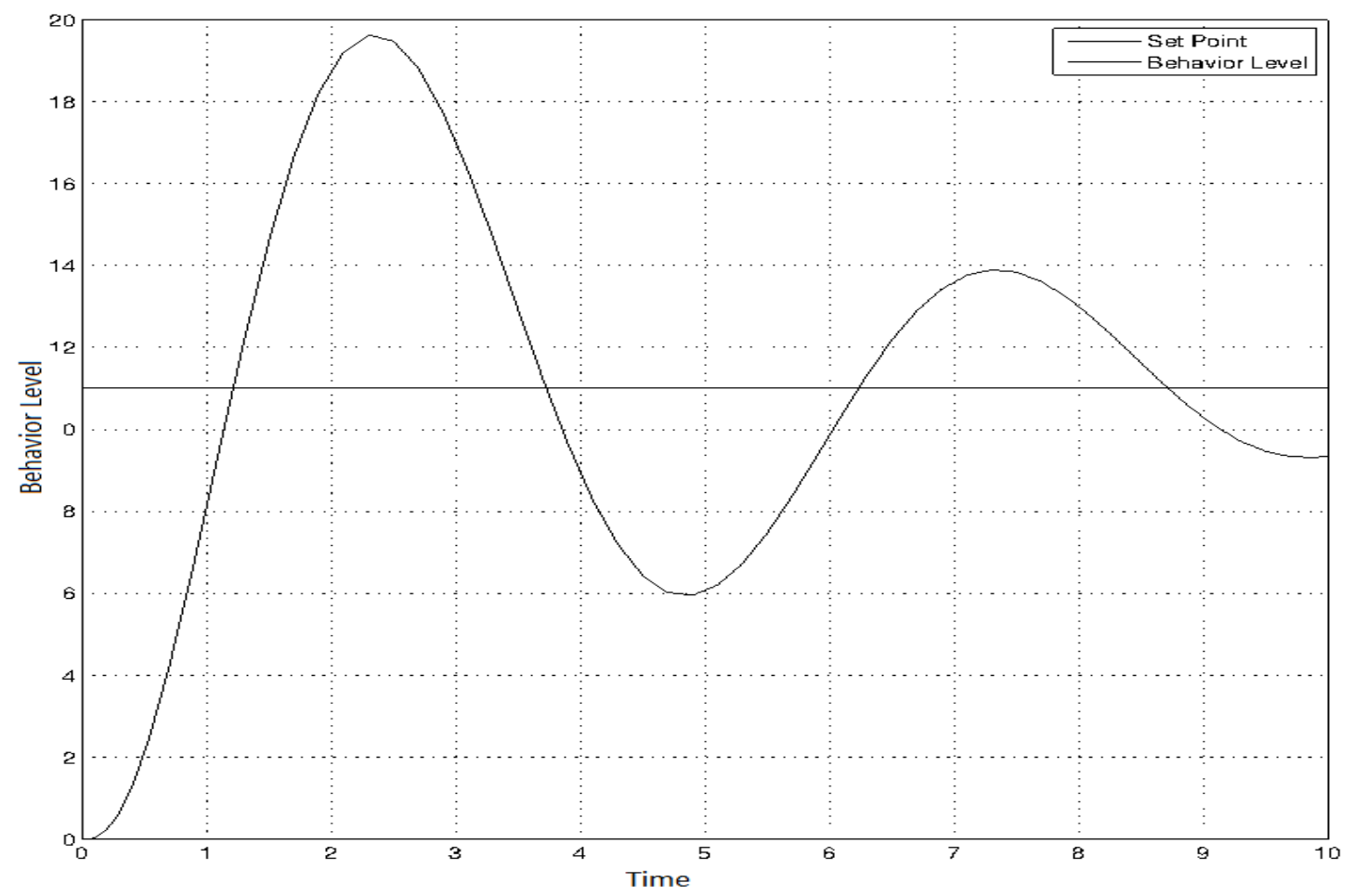

Figure 4.16: The response of the individual's Behavioral level for a step change of 11 when Behavior belief $\left(\xi_{1}\right)$ is used as controlled variable.

\subsection{Client-Therapist Relation Using The Social Cognitive Theory}

In this section, the client's side is extended based on the social cognitive theory. In the literature, there are two different human behavior models that were proposed based on the SCT. Fluid analogy model was proposed in [3] to describe the standard social cognitive theory; which consists of six state variables as shown in Figure 4.17, while [7] proposed fluid model for the simplified social cognitive theory; in which only four state variable were considered as demonstrated in Figure 4.18. 


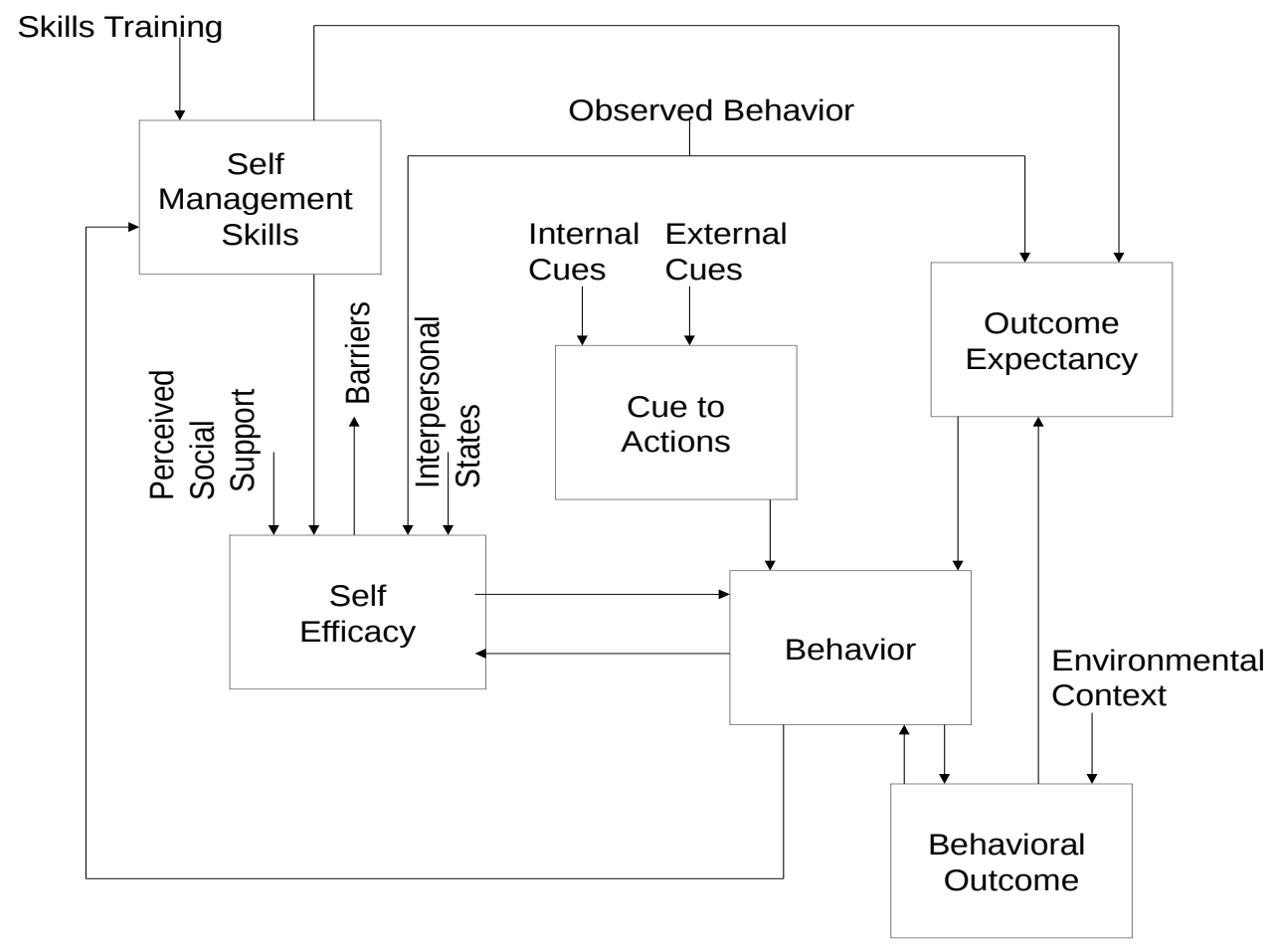

Figure 4.17: Block diagram of the Social Cognitive Theory. 


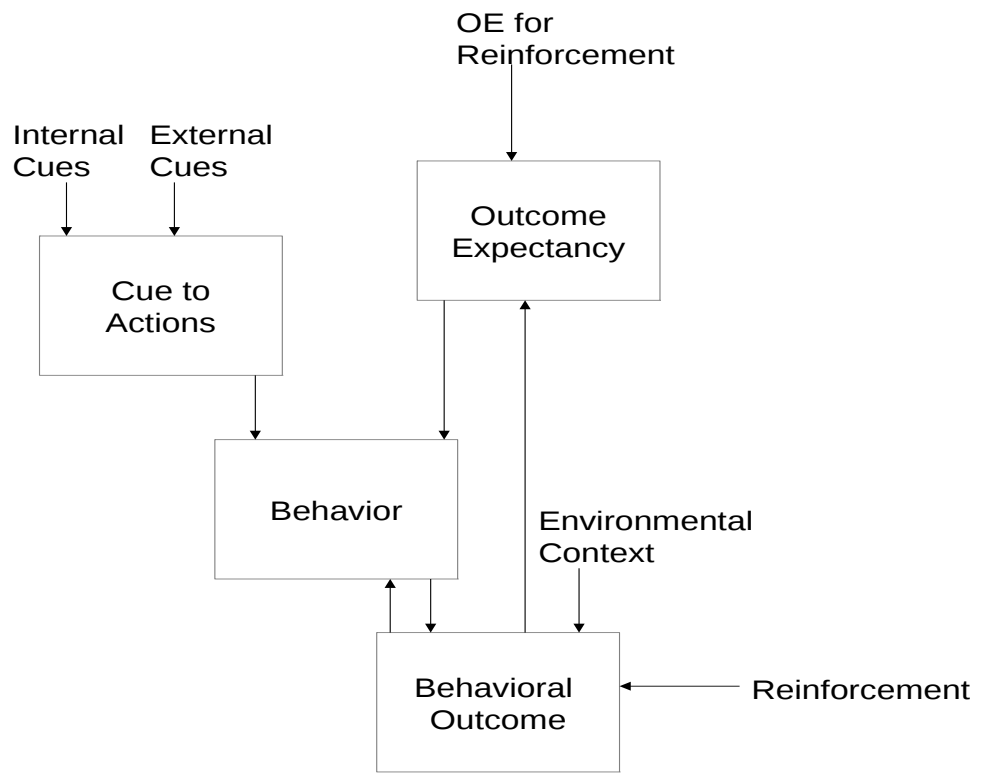

Figure 4.18: Block diagram of the simplified Social Cognitive Theory.

The major constructs of SCT, shown in Figure 4.17 are Self-management Skills $\left(\eta_{1}\right)$, Outcome Expectancy $\left(\eta_{2}\right)$, Self-Efficacy $\left(\eta_{3}\right)$, Behavior $\left(\eta_{4}\right)$, Behavioral Outcomes $\left(\eta_{5}\right)$ and Cue to Actions $\left(\eta_{6}\right)$. These constructs are represented as storage elements in the Bond Graph model shown in Figure 4.19. These elements are the six state variables in the model. The input sources are Skills training $\left(\xi_{1}\right)$, Observed Behavior $\left(\xi_{2}\right)$, Perceived Social Support $\left(\xi_{1}\right)$, Internal Cues $\left(\xi_{4}\right)$, Perceived Barriers $\left(\xi_{5}\right)$, Interpersonal States $\left(\xi_{6}\right)$, Environmental Context $\left(\xi_{7}\right)$, External Cues $\left(\xi_{8}\right)$; these are modeled as sources of flow in the Bond Graph. The uncertainties associated with the state variables are modeled as disturbances and all these are also modeled as sources of flow $\left(\varsigma_{1}, \varsigma_{2}, \cdots, \varsigma_{6}\right)$ in the Bond Graph model. 
The simplified SCT excludes the Self-management Skills $\left(\eta_{1}\right)$ and the SelfEfficacy $\left(\eta_{3}\right)$. It only includes three input sources out of the original eight inputs which are, Internal Cues $\left(\xi_{4}\right)$, Environmental Context $\left(\xi_{7}\right)$ and External Cues $\left(\xi_{8}\right)$; in addition to two new input sources: Outcome Expectancy reinforcement $\left(\xi_{9}\right)$ and Reinforcement $\left(\xi_{1} 0\right)$. The Bond Graph representation for the simplified social cognitive theory is shown in Figure 4.20. 


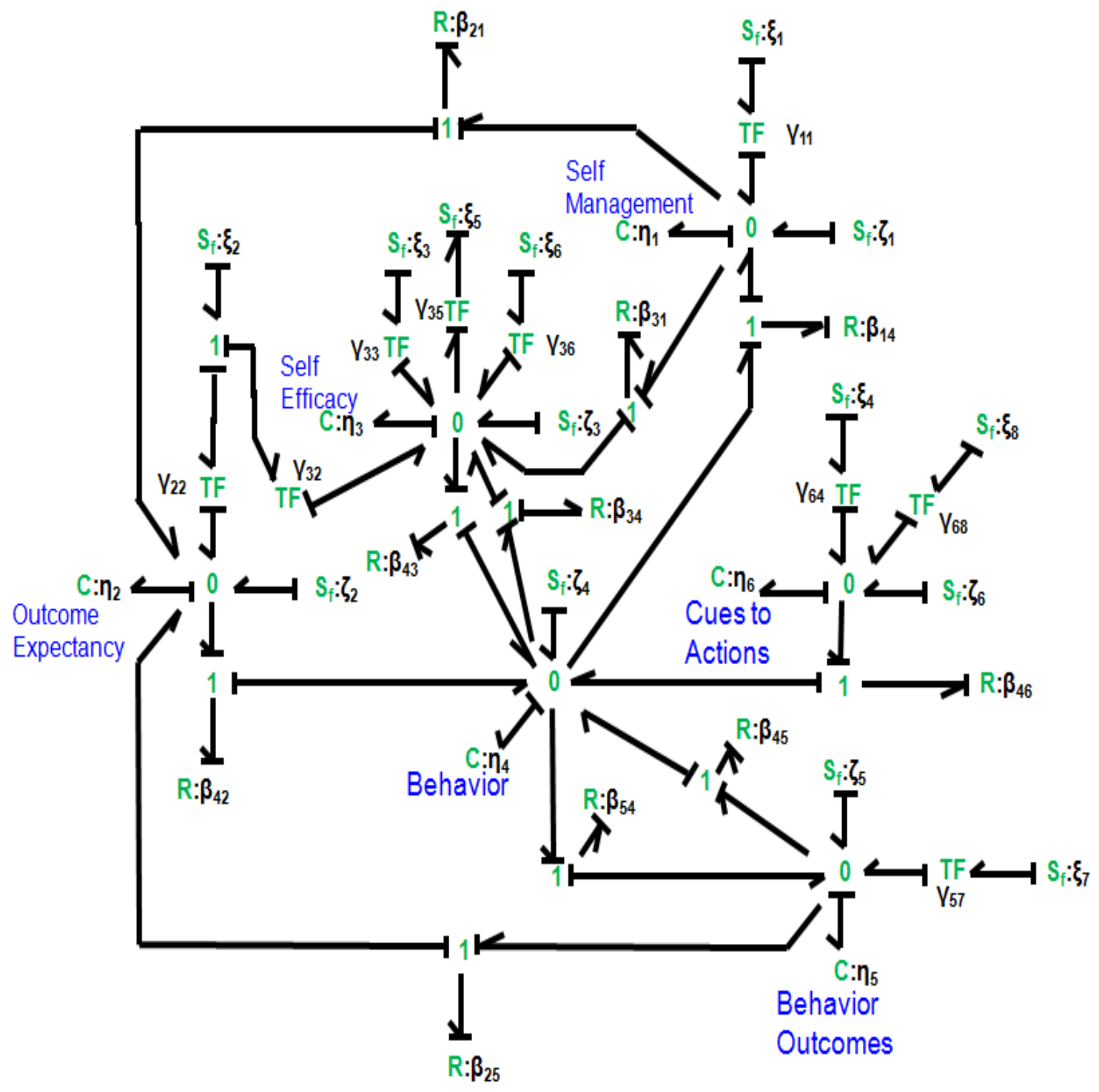

Figure 4.19: Bond graph model based on the Social Cognitive Theory. 


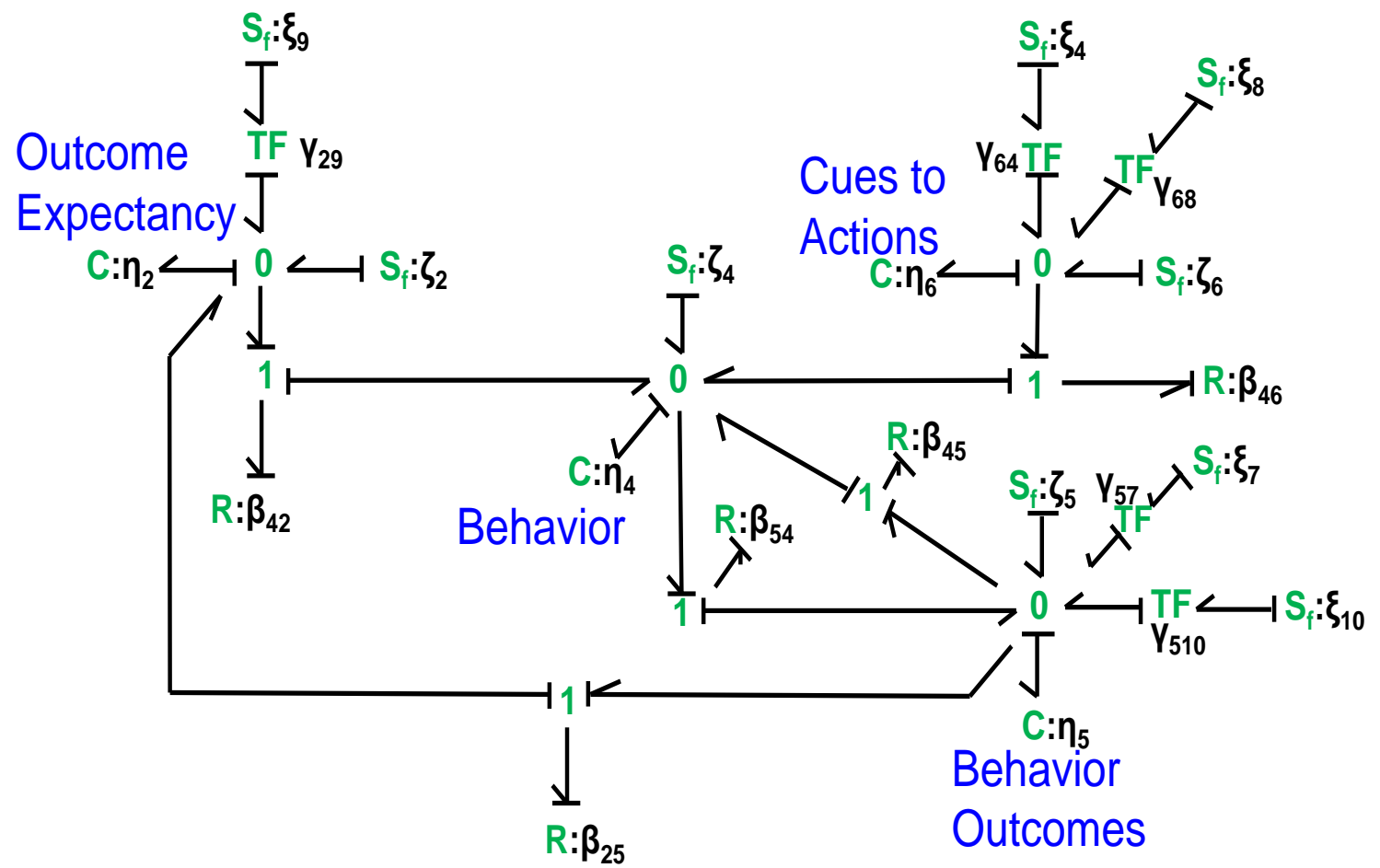

Figure 4.20: Bond graph model based on the Simplified Social Cognitive Theory.

The dynamic equations obtained using Bond Graph for the SCT are

$$
\frac{d \eta_{1}}{d t}=\gamma_{11} \xi_{1}+\frac{1}{\beta_{41}}\left(\eta_{1}-\eta_{4}\right)-\frac{1}{\beta_{21}}\left(\eta_{1}-\eta_{2}\right)-\frac{1}{\beta_{31}}\left(\eta_{1}-\eta_{3}\right)+\varsigma_{1}
$$

$$
\frac{d \eta_{2}}{d t}=\gamma_{22} \xi_{2}+\frac{1}{\beta_{25}}\left(\eta_{5}-\eta_{2}\right)+\frac{1}{\beta_{21}}\left(\eta_{1}-\eta_{2}\right)-\frac{1}{\beta_{42}}\left(\eta_{2}-\eta_{4}\right)+\varsigma_{2}
$$




$$
\frac{d \eta_{3}}{d t}=\gamma_{33} \xi_{3}+\gamma_{36} \xi_{3}-\gamma_{35} \xi_{5}+\frac{1}{\beta_{31}}\left(\eta_{1}-\eta_{3}\right)+\frac{1}{\beta_{34}}\left(\eta_{4}-\eta_{3}\right)-\frac{1}{\beta_{43}}\left(\eta_{3}-\eta_{4}\right)+\varsigma_{3}
$$

$$
\begin{gathered}
\frac{d \eta_{4}}{d t}=\frac{1}{\beta_{46}}\left(\eta_{6}-\eta_{4}\right)+\frac{1}{\beta_{42}}\left(\eta_{2}-\eta_{4}\right)+\frac{1}{\beta_{45}}\left(\eta_{5}-\eta_{4}\right) \\
+\frac{1}{\beta_{43}}\left(\eta_{3}-\eta_{4}\right)-\frac{1}{\beta_{54}}\left(\eta_{4}-\eta_{5}\right)-\frac{1}{\beta_{14}}\left(\eta_{4}-\eta_{1}\right)-\frac{1}{\beta_{34}}\left(\eta_{4}-\eta_{3}\right)+\varsigma_{4} \\
\frac{d \eta_{5}}{d t}=\gamma_{57} \xi_{7}+\frac{1}{\beta_{54}}\left(\eta_{4}-\eta_{5}\right)-\frac{1}{\beta_{45}}\left(\eta_{5}-\eta_{4}\right)-\frac{1}{\beta_{25}}\left(\eta_{5}-\eta_{2}\right)+\varsigma_{5} \\
\frac{d \eta_{6}}{d t}=\gamma_{64} \xi_{4}+\gamma_{68} \xi_{8}-\frac{1}{\beta_{46}}\left(\eta_{6}-\eta_{4}\right)+\varsigma_{6}
\end{gathered}
$$

The dynamic equations for the simplified model are

$$
\frac{d \eta_{2}}{d t}=\gamma_{29} \xi_{9}+\frac{1}{\beta_{25}}\left(\eta_{5}-\eta_{2}\right)-\frac{1}{\beta_{42}}\left(\eta_{2}-\eta_{4}\right)+\varsigma_{2}
$$




$$
\frac{d \eta_{4}}{d t}=\frac{1}{\beta_{46}}\left(\eta_{6}-\eta_{4}\right)+\frac{1}{\beta_{42}}\left(\eta_{2}-\eta_{4}\right)+\frac{1}{\beta_{45}}\left(\eta_{5}-\eta_{4}\right)-\frac{1}{\beta_{54}}\left(\eta_{4}-\eta_{5}\right)+\varsigma_{4}
$$

$$
\frac{d \eta_{5}}{d t}=\gamma_{57} \xi_{7}+\gamma_{510} \xi_{10}+\frac{1}{\beta_{54}}\left(\eta_{4}-\eta_{5}\right)-\frac{1}{\beta_{45}}\left(\eta_{5}-\eta_{4}\right)-\frac{1}{\beta_{25}}\left(\eta_{5}-\eta_{2}\right)+\varsigma_{5}
$$

$$
\frac{d \eta_{6}}{d t}=\gamma_{64} \xi_{4}+\gamma_{68} \xi_{8}-\frac{1}{\beta_{46}}\left(\eta_{6}-\eta_{4}\right)+\varsigma_{6}
$$

Now, the Client's tank from the simple two tanks diagram shown in Figure 4.1 is replaced by the block diagrams of the SCT and SSCT shown in Figures 4.17 and 4.18. The overall block diagrams are shown in Figures 4.21 and 4.22. 


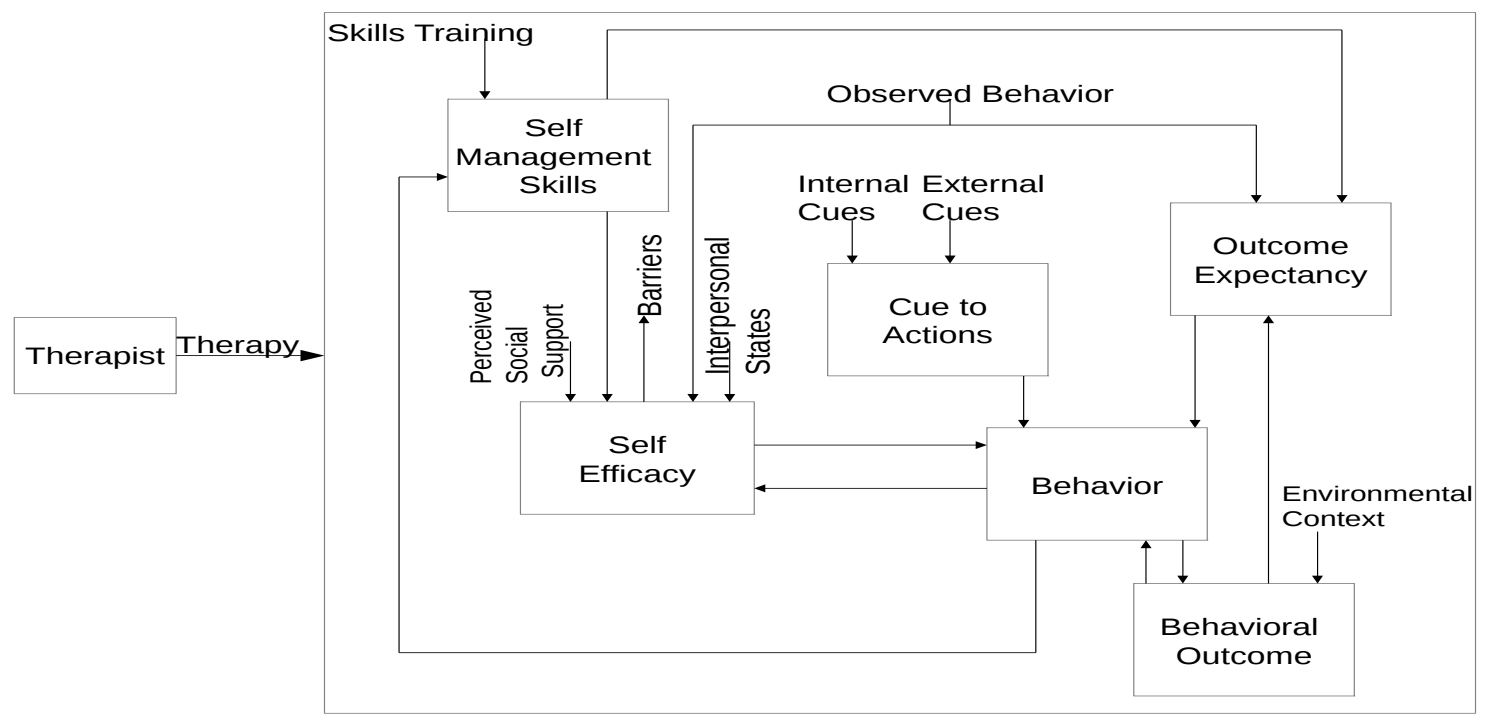

Figure 4.21: Client-Therapist block diagram based on the Social Cognitive Theory.

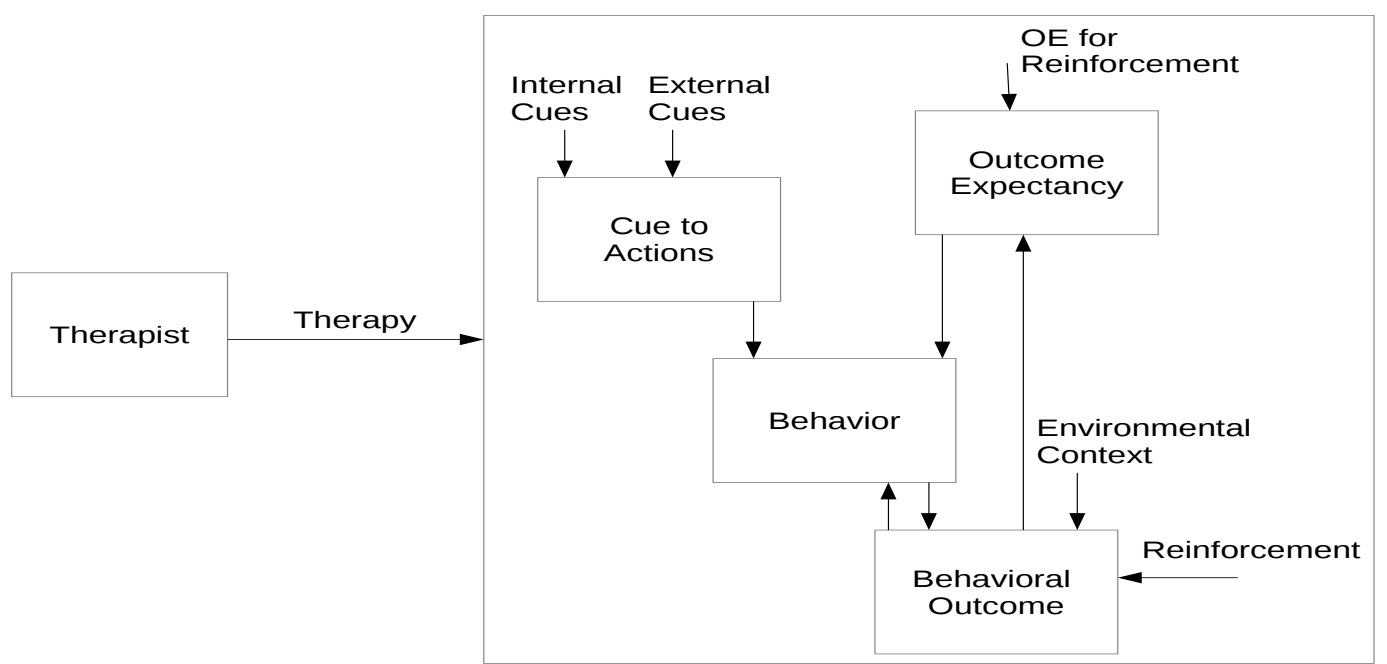

Figure 4.22: Client-Therapist block diagram based on the simplified Social Cognitive Theory. 
The Bond Graph of these two Client-Therapist models are constructed and shown in Figures 4.23and 4.24.

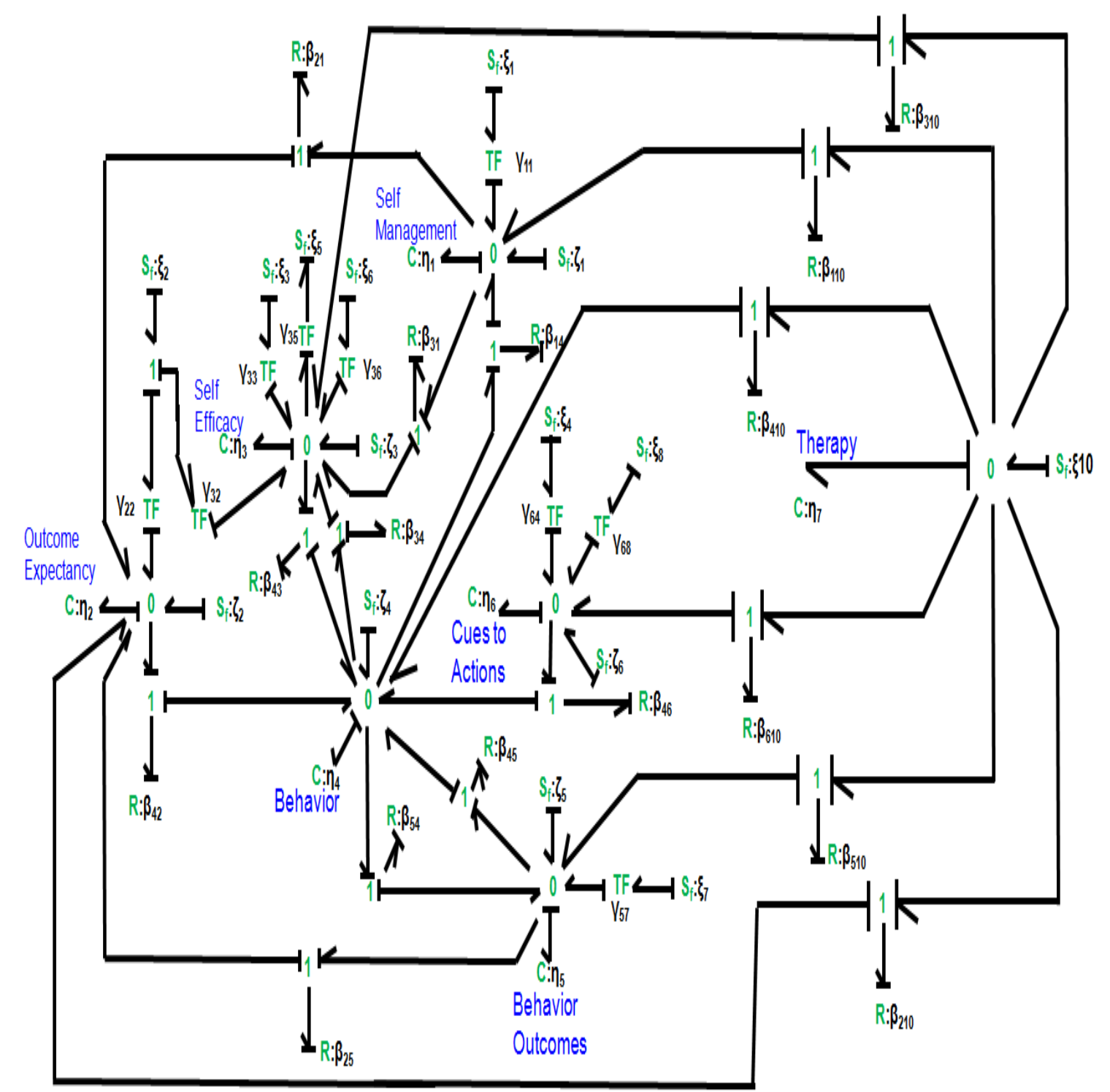

Figure 4.23: Bond graph for the Client-Therapist model based on the Social Cognitive Theory. 


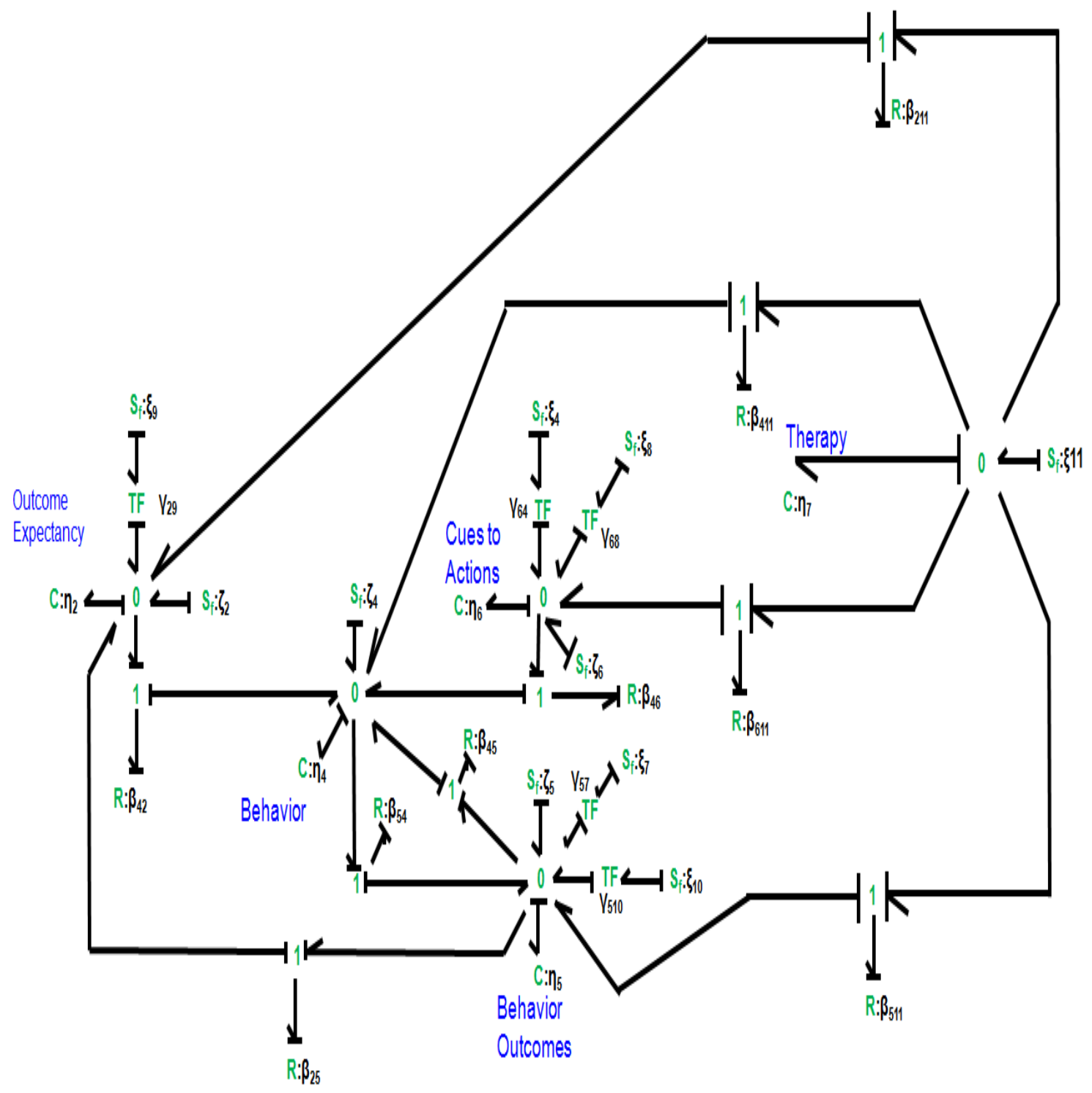

Figure 4.24: Bond graph for the Client-Therapist model based on the simplified Social Cognitive Theory.

\subsubsection{State-Space Representation}

The same steps as described in the previous section are followed to obtain the state space representation for the social cognitive theory model (SCT) and the simplified social cognitive theory model (SSCT). 
The SCT state vector is

$$
X=\left[\begin{array}{llllll}
\eta_{1} & \eta_{2} & \eta_{3} & \eta_{4} & \eta_{5} & \eta_{6}
\end{array}\right]{ }^{T}
$$

SCT state change vector is

$$
\frac{d X}{d t}=\left[\begin{array}{llllll}
\frac{d \eta_{1}}{d t} & \frac{d \eta_{2}}{d t} & \frac{d \eta_{3}}{d t} & \frac{d \eta_{4}}{d t} & \frac{d \eta_{5}}{d t} & \frac{d \eta_{6}}{d t}
\end{array}\right]^{T}
$$

SCT input vector

$U=\left[\begin{array}{llllllllllllll}\xi_{1} & \xi_{2} & \xi_{3} & \xi_{4} & \xi_{5} & \xi_{6} & \xi_{7} & \xi_{8} & \zeta_{1} & \zeta_{2} & \zeta_{3} & \zeta_{4} & \zeta_{5} & \zeta_{6}\end{array}\right]^{T}$

SCT State matrix

$A=\left[\begin{array}{cccccc}-\frac{1}{\beta_{14}}-\frac{1}{\beta_{21}}-\frac{1}{\beta_{31}} & \frac{1}{\beta_{21}} & \frac{1}{\beta_{31}} & \frac{1}{\beta_{14}} & 0 & 0 \\ \frac{1}{\beta_{21}} & -\frac{1}{\beta_{21}}-\frac{1}{\beta_{25}}-\frac{1}{\beta_{42}} & 0 & \frac{1}{\beta_{42}} & \frac{1}{\beta_{25}} & 0 \\ \frac{1}{\beta_{31}} & 0 & -\frac{1}{\beta_{31}}-\frac{1}{\beta_{34}}-\frac{1}{\beta_{43}} & \frac{1}{\beta_{34}}+\frac{1}{\beta_{43}} & 0 & 0 \\ \frac{1}{\beta_{14}} & \frac{1}{\beta_{42}} & \frac{1}{\beta_{34}}+\frac{1}{\beta_{43}}-\frac{1}{\beta_{14}}-\frac{1}{\beta_{42}}-\frac{1}{\beta_{34}}-\frac{1}{\beta_{43}}-\frac{1}{\beta_{45}}-\frac{1}{\beta_{54}}-\frac{1}{\beta_{44}} \frac{1}{\beta_{45}}+\frac{1}{\beta_{54}} & \frac{1}{\beta_{46}} & \\ 0 & \frac{1}{\beta_{25}} & 0 & \frac{1}{\beta_{45}}+\frac{1}{\beta_{54}}-\frac{1}{\beta_{25}}-\frac{1}{\beta_{45}}-\frac{1}{\beta_{54}} & 0 \\ 0 & 0 & 0 & \frac{1}{\beta_{46}} & 0 & -\frac{1}{\beta_{46}}\end{array}\right]$

SCT input matrix

$$
B=[\gamma \mid \zeta]
$$




$$
\gamma=\left[\begin{array}{cccccccc}
\gamma_{11} & 0 & 0 & 0 & 0 & 0 & 0 & 0 \\
0 & \gamma_{22} & 0 & 0 & 0 & 0 & 0 & 0 \\
0 & \gamma_{32} & \gamma_{33} & 0 & \gamma_{35} & \gamma_{36} & 0 & 0 \\
0 & 0 & 0 & 0 & 0 & 0 & 0 & 0 \\
0 & 0 & 0 & 0 & 0 & 0 & \gamma_{57} & 0 \\
0 & 0 & 0 & \gamma_{64} & 0 & 0 & 0 & \gamma_{68}
\end{array}\right]
$$

SCT output matrix

$$
C=\left[\begin{array}{llllll}
0 & 0 & 0 & 1 & 0 & 0
\end{array}\right]
$$

SCT feed through matrix

$$
D=z \operatorname{eros}(1,14)
$$

While the state space representation for the simplified social cognitive theory is obtained as follows: 
SSCT state vector

$$
X=\left[\begin{array}{llll}
\eta_{2} & \eta_{4} & \eta_{5} & \eta_{6}
\end{array}\right]{ }^{T}
$$

SSCT state change vector

$$
\frac{d X}{d t}=\left[\begin{array}{llll}
\frac{d \eta_{2}}{d t} & \frac{d \eta_{4}}{d t} & \frac{d \eta_{5}}{d t} & \frac{d \eta_{6}}{d t}
\end{array}\right]^{T}
$$

SSCT input vector

$$
U=\left[\begin{array}{lllllllll}
\xi_{9} & \xi_{7} & \xi_{10} & \xi_{4} & \xi_{8} & \zeta_{2} & \zeta_{4} & \zeta_{5} & \zeta_{6}
\end{array}\right]^{T}
$$

SSCT state matrix

$$
A=\left[\begin{array}{cccc}
-\frac{1}{\beta_{25}}-\frac{1}{\beta_{42}} & \frac{1}{\beta_{42}} & \frac{1}{\beta_{25}} & 0 \\
\frac{1}{\beta_{42}} & -\frac{1}{\beta_{42}}-\frac{1}{\beta_{45}}-\frac{1}{\beta_{54}}-\frac{1}{\beta_{46}} & \frac{1}{\beta_{45}}+\frac{1}{\beta_{54}} & \frac{1}{\beta_{46}} \\
\frac{1}{\beta_{25}} & \frac{1}{\beta_{45}}+\frac{1}{\beta_{54}} & -\frac{1}{\beta_{25}}-\frac{1}{\beta_{45}}-\frac{1}{\beta_{54}} & 0 \\
0 & \frac{1}{\beta_{46}} & 0 & -\frac{1}{\beta_{46}}
\end{array}\right]
$$


SSCT input matrix

$$
B=\left[\begin{array}{ccccccccc}
\gamma_{29} & 0 & 0 & 0 & 0 & 1 & 0 & 0 & 0 \\
0 & 0 & 0 & 0 & 0 & 0 & 1 & 0 & 0 \\
0 & \gamma_{57} & \gamma_{510} & 0 & 0 & 0 & 0 & 1 & 0 \\
0 & 0 & 0 & \gamma_{64} & \gamma_{68} & 0 & 0 & 0 & 0
\end{array}\right]
$$

SSCT output matrix

$$
C=\left[\begin{array}{llll}
0 & 1 & 0 & 0
\end{array}\right]
$$

SSCT feed through matrix

$$
D=\left[\begin{array}{lllllllll}
0 & 0 & 0 & 0 & 0 & 0 & 0 & 0 & 0
\end{array}\right]
$$

To calculate the transfer function of these two MIMO system the following equation is used

$$
H(s)=C \times\left((s * I-A)^{-1}\right) \times B+D
$$

\subsubsection{Stability Analysis and Bode Plots}

In order to perform stability analysis, unit step values were set for all the input sources, and unit ratios for the transformers, while the value of $50 \%$ was selected for the resistor elements. Root Locus analysis was carried out for both transfer functions 
and it showed that, both systems are critical stable, hence more analysis is required to determine the gain and phase margins for each input.

The Bode plots for the two systems are generated and shown in Figures 4.25 and 4.26 .

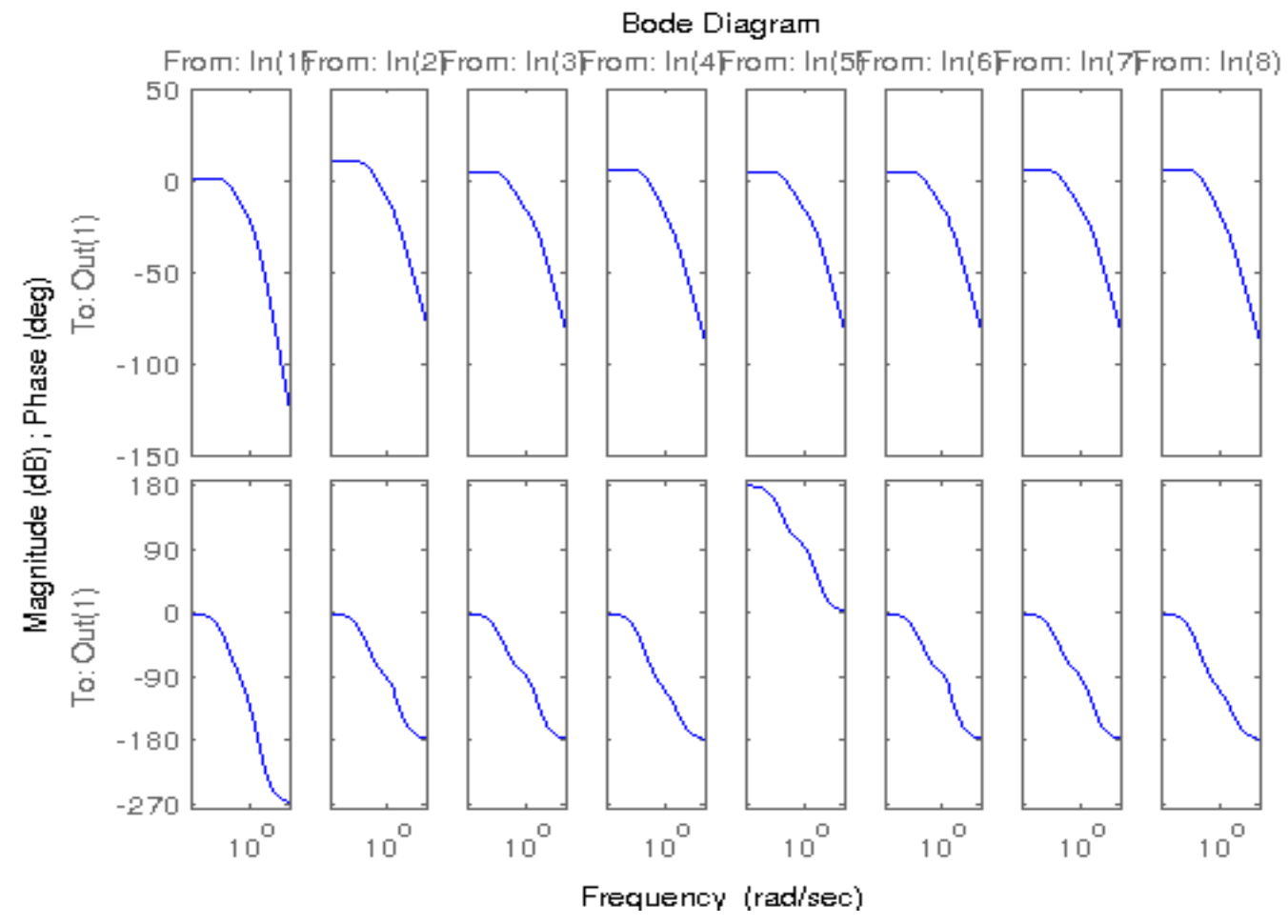

Figure 4.25: Bode plots for the Client-Therapist model based on the Social Cognitive Theory. 


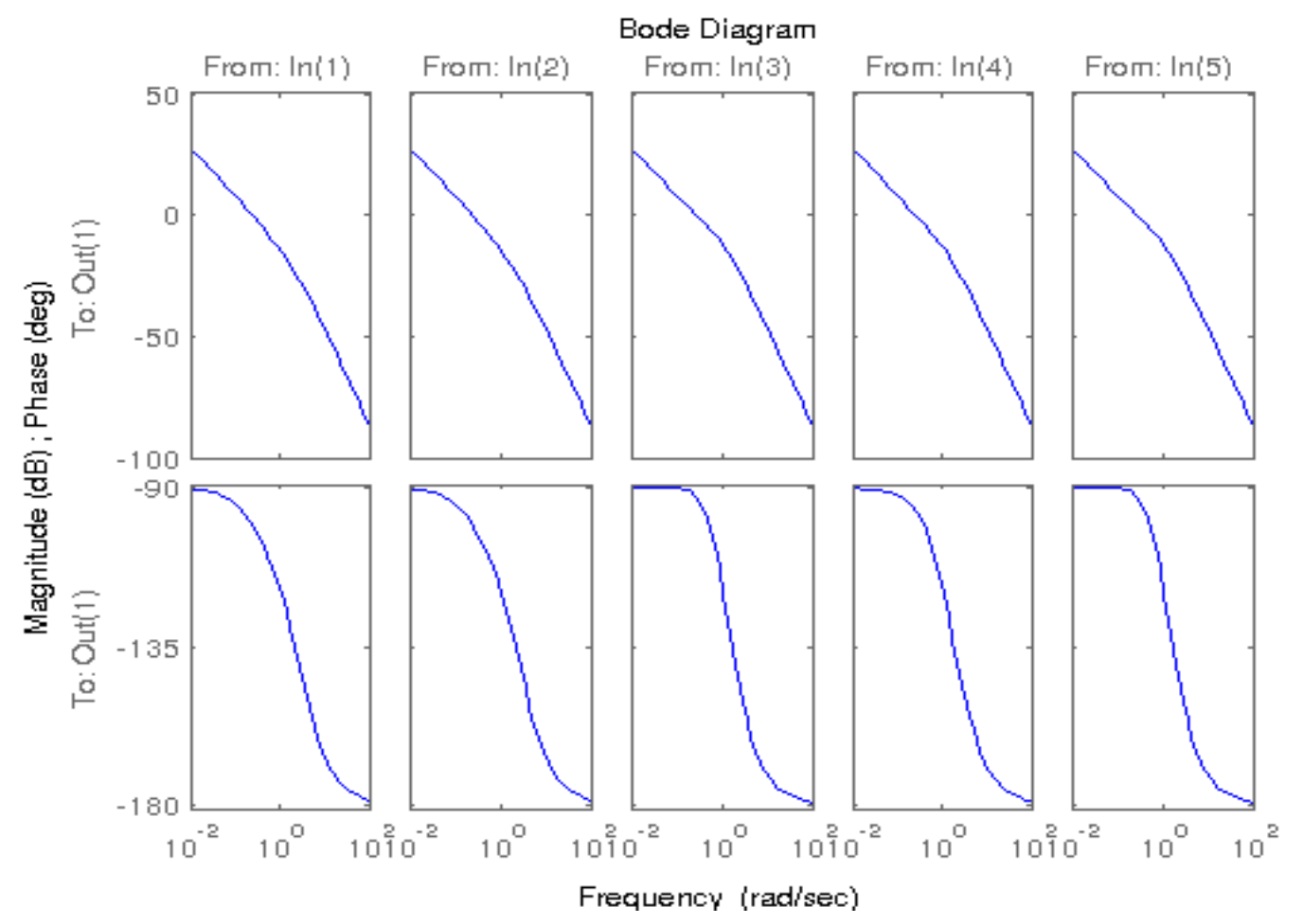

Figure 4.26: Bode plots for the Client-Therapist model based on the simplified Social Cognitive Theory.

Notice that the simplified models include the higher margin inputs; this means that, the variables those were removed due to the simplification process are not significant. It can be concluded that all of the five input variables in the simplified model have infinite gain margin. This means that any of them can manipulated to enhance the response. However, the phase margin indicates that the best option to be controlled is the Reinforcement input source which directly affect the behavioral outcome storage element.

To generalize this conclusion the system was evaluated under different values for the three resistor elements $\beta_{42}, \beta_{45}$ and $\beta_{46}$, since these are the only direct resistor 
elements connected to the behavior storage element $\eta_{4}$. Two different values were considered for each resister element, low resistance(10\%) and high resistance (90\%). Thus the total number of combinations to be examined is eight. For each combinations Bode plots were generated to capture the gain and phase margins, then the input variable with the highest margins was selected as the best manipulated variable. Figure 4.27 shows the results.

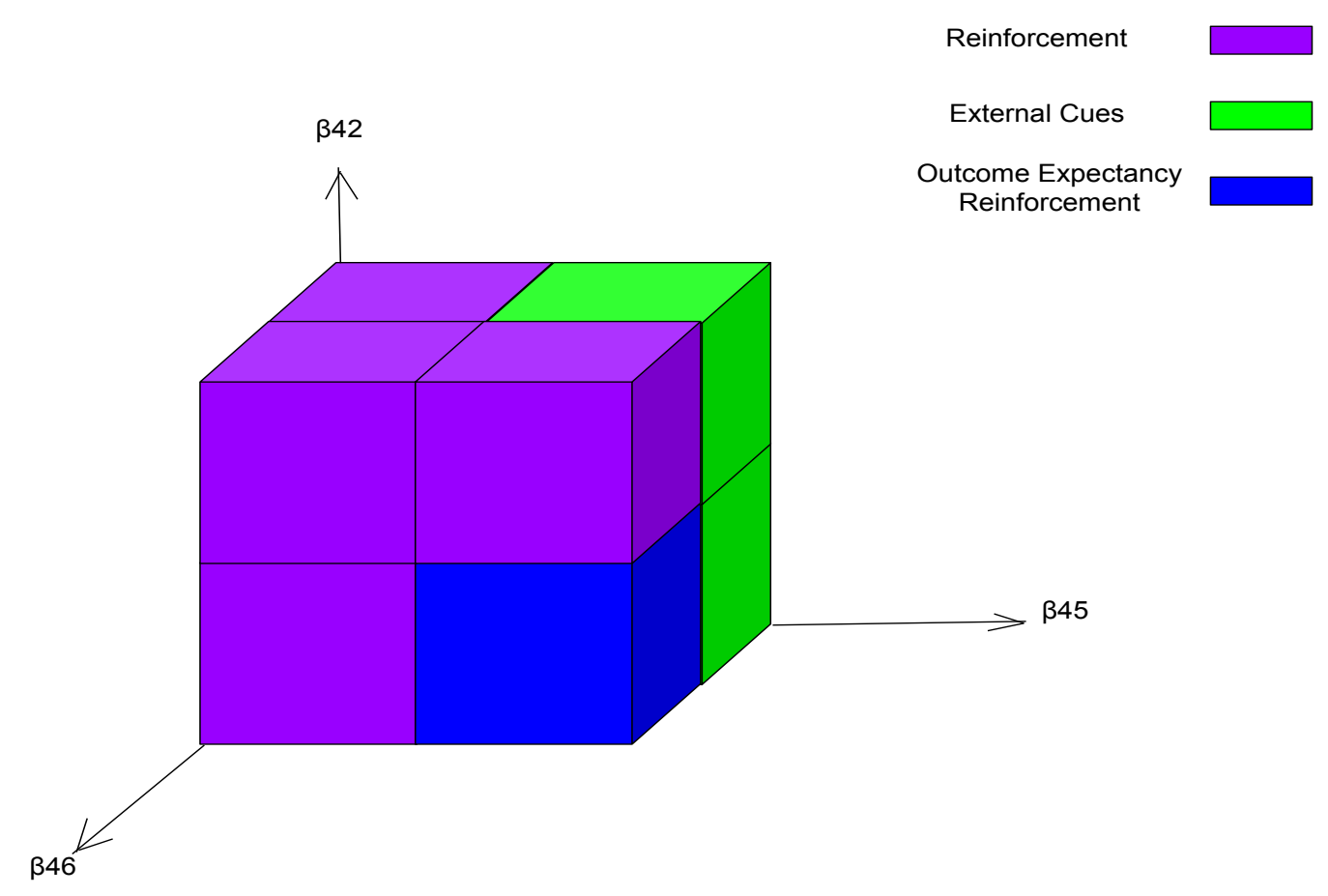

Figure 4.27: Plot shows the best input variable to be chosen as manipulated variable in each combination of resistances values in the simplified Social Cognitive Theory model. Notice that, the Reinforcement is recommended in five out of the eight regions

The Bond Graph approach presented here to model human behavior is promising. Starting from a basic model to describe the interaction between a client 
and a therapist, it was demonstrated that, the Bond Graph approach can be extended to include well known theories e.g. the theory of planned behavior and the social cognitive theory. The approach then yields dynamic models that were used for control application to improve the interaction. Stability analysis was carried out for different models in order to point out the gain and phase margins for the inputs of every system, which leads to choosing the best manipulated variable in order to achieve the control goals. 


\section{CHAPTER V}

\section{DIAGNOSIS OF HUMAN BEHAVIOR USING BOND GRAPH AND}

\section{ANALYTICAL REDUNDANCY RELATIONS}

The approach of using the Bond Graph method for human behavior models was introduced in Chapter 3 and Chapter 4 introduced a basis for designing controllers to support the Client-Therapist interaction. This Chapter proposes a robust diagnosis scheme, that can be utilized to continuously monitor t he Client-Therapist interaction and provide insights and guidance in case of unexpected observed behavior.

Abnormal behavior in any system is identified when the value of one or more of the output variables of the system diverges from the expected steady state value. This divergence from the steady state value leads to a transient state which will eventually take the system to a different steady state. According to the severity of this deviation from the expected state, the new state will be determined, sometimes rapid divergence can lead to complete failure of the system, hence it is very important to have continuous monitoring, analysis and diagnosis for the underlying system to mitigate the failures. The expected behavior of the system is obtained using an underlying model for the system. 
The Fault Detection and Isolation (FDI) technique is well-known and used successfully in many systems. FDI guides the search process to identify the source of the fault after an unexpected output is recognized. In the context of human behavior models, the same principle applies. A tool for monitoring the behavior is required. This tool must detect when an abnormal behavior is observed. The objective of FDI then is to identify the source that has led to this abnormal behavior in order to mitigate any harm that can be caused by this behavior.

In this Chapter the well-known Analytical Redundancy Relation (ARR) approach [54] is adopted and implemented on human behavior models that were proposed in earlier Chapters.

The basic idea of ARR is to compare the actual behavior of the system with the behavior of a reference model, that is done, by observing the outputs of the system and comparing them to those of the reference model when both are subjected to the same set of inputs. The difference of this comparison is sent to the FDI module which will identify the source of the fault based on predefined conditions and relations between the outputs and the different components of the system.

\subsection{Analysis and Fault Detection}

Fault detection is achieved by comparing the behavior of a system under normal and faulty operation scenarios. Under normal operation conditions, the instantaneous values of all the parameters should be within specified bounds. When the values of 
observed variables or parameters exceed an acceptable range then a fault is assumed to have occurred. The following four steps are necessary to diagnose any fault [74]:

- Detection,

- Isolation,

- Identification, and

- Estimation.

The detection stage is focused on determining whether fault has occurred. The isolation stage is focused on locating the source of the fault. The identification step is focused on finding the type of the fault. The estimation stage will determine the severity of the fault.

Faults that occur in physical systems can be classified according to the source of the failure as [74]:

- Parametric faults.

- Structural faults.

- Instrumentation faults.

Parametric faults occur due to a change in one of the system's parameter value. For instance, a blockage of a valve will result in a change of the flow rate of a pipe. A structural fault may happen because of a failure in one of the components, for example, a broken electrical switch or a broken mechanical device. Instruments 
may also fail due to external environmental factors such as temperature, or internal factors such as unstable power supply.

Faults can also be classified based on their severeness and occurrence into four categories:

- Abrupt faults - that occur suddenly for any component and takes the operation to the abnormal range.

- Progressive faults - where the behavior deviates from the expected values gradually.

- Incipient faults - where the measurements are incorrect and yet in the normal range; usually happen because of the wear and tear.

- Intermittent faults - where abnormal behaviors persist for a short time intervals. Examples for different types of faults with the human behavior models are presented in Table 5.1.

Fault detection and isolation (FDI) are critical steps in fault diagnosis. There are many FDI techniques and methods that can be classified into the following three categories:

- Process history based methods that rely on an analysis of huge amounts of measured data to find properties of faults, their frequencies and severity.

- Qualitative model-based methods that focus on identifying important cause and effect relations. 
Table 5.1: Physical examples and human behavior examples for different fault types

\begin{tabular}{|c|c|c|}
\hline Fault Type & Physical Example & Human Behavior Example \\
\hline Parametric faults & Blockage of a pipe & $\begin{array}{l}\text { An increase in the therapy } \\
\text { resistance as a result of not } \\
\text { meeting the expected results }\end{array}$ \\
\hline Structural faults & Broken electrical switch & $\begin{array}{l}\text { Failure in transforming } \\
\text { the intention into behavior } \\
\text { due to memory problem }\end{array}$ \\
\hline Instrumentation faults & Temperature sensor failure & $\begin{array}{l}\text { Failure in the method of } \\
\text { measuring the intention }\end{array}$ \\
\hline Abrupt faults & $\begin{array}{l}\text { Sudden current increase } \\
\text { in electric circuit }\end{array}$ & $\begin{array}{l}\text { Change in the expected } \\
\text { behavior due to a shock } \\
\text { or unexpected news }\end{array}$ \\
\hline Progressive faults & Leakage of bearings lubrication & $\begin{array}{c}\text { Failure in following } \\
\text { the recommended diet } \\
\text { by the nutrition expert }\end{array}$ \\
\hline Incipient faults & Battery capacity & $\begin{array}{c}\text { The resistance to perceived } \\
\text { behavioral control may } \\
\text { very due to the daily mood }\end{array}$ \\
\hline Intermittent faults & $\begin{array}{l}\text { borderline electrical connection } \\
\text { in the wiring where } \\
\text { two conductors may touch }\end{array}$ & $\begin{array}{c}\text { The resistance for social } \\
\text { norms may change when } \\
\text { around some friends }\end{array}$ \\
\hline
\end{tabular}

- Quantitative model-based methods that exploit mathematical relations between the different variables, outputs and inputs of the system.

In this Chapter a quantitative model-based technique for the analysis and diagnosis of the human behavior models using Bond Graphs is presented. ARRs were used compare actual observed behavior with what is expected through the model. The key idea is to find residuals that can guide the detection and isolation of faults.

Figure 5.1 illustrates the scheme by which ARRs are useful. The same inputs are applied both to the actual system and the model. The differences observed 
between the two outputs are denoted as errors; these are also known as residuals. The FDI methods rely on these residuals to detect and isolate the faults. An FDI module identifies the source of the fault based on predefined conditions and relations between the outputs and the different components of the system.

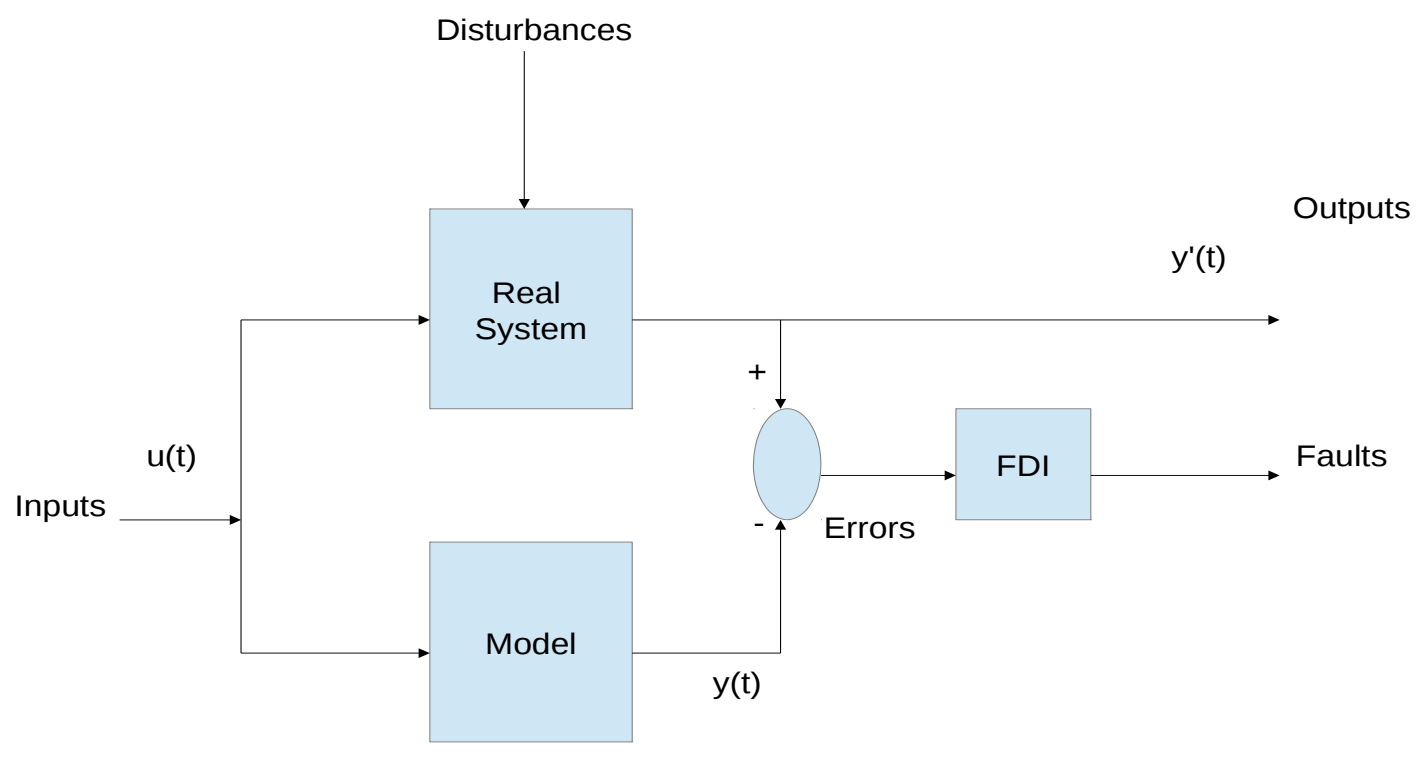

Figure 5.1: Block diagram illustrates the Analytical Redundancy Relation technique for system diagnosis

The main contribution of this Chapter is the design of the FDI module for detecting and isolating variations ("faults") in human behavior. The starting point is a Bond Graph model for the expected behavior. Dynamic equations that describe the behavior from the Bond Graph model are derived as in previous Chapters and the outputs are expressed in terms of the system parameters. The relations of each 
of these parameters to the outputs are determined. For example, when a specific parameter is increased, does a particular output variable increase or decrease? Is the relation proportional? If the output remains constant, does this mean that the parameter is not related to the output?

For each of the outputs, the normal operational range for the values is predefined. When an error occurs, i.e., the difference between an expected value and observed value exceeds the normal range, the FDI module has to report the set of possible sources of the fault. This set is determined according to the relations calculated after the model was designed. Finally, an extra analysis is needed to identify the exact fault source out of the set of possible sources, this analysis is based on the physical characteristics of the components; for example, some parameter values can only increase due to a fault (like the resistance of a pipe to the flow of a fluid), or only decrease (like the capacity of a storage tank), and some parameters have the ability to both increase or decrease due to a failure.

The steps of the proposed FDI for the human behavior models are illustrated in Figure 5.2. 


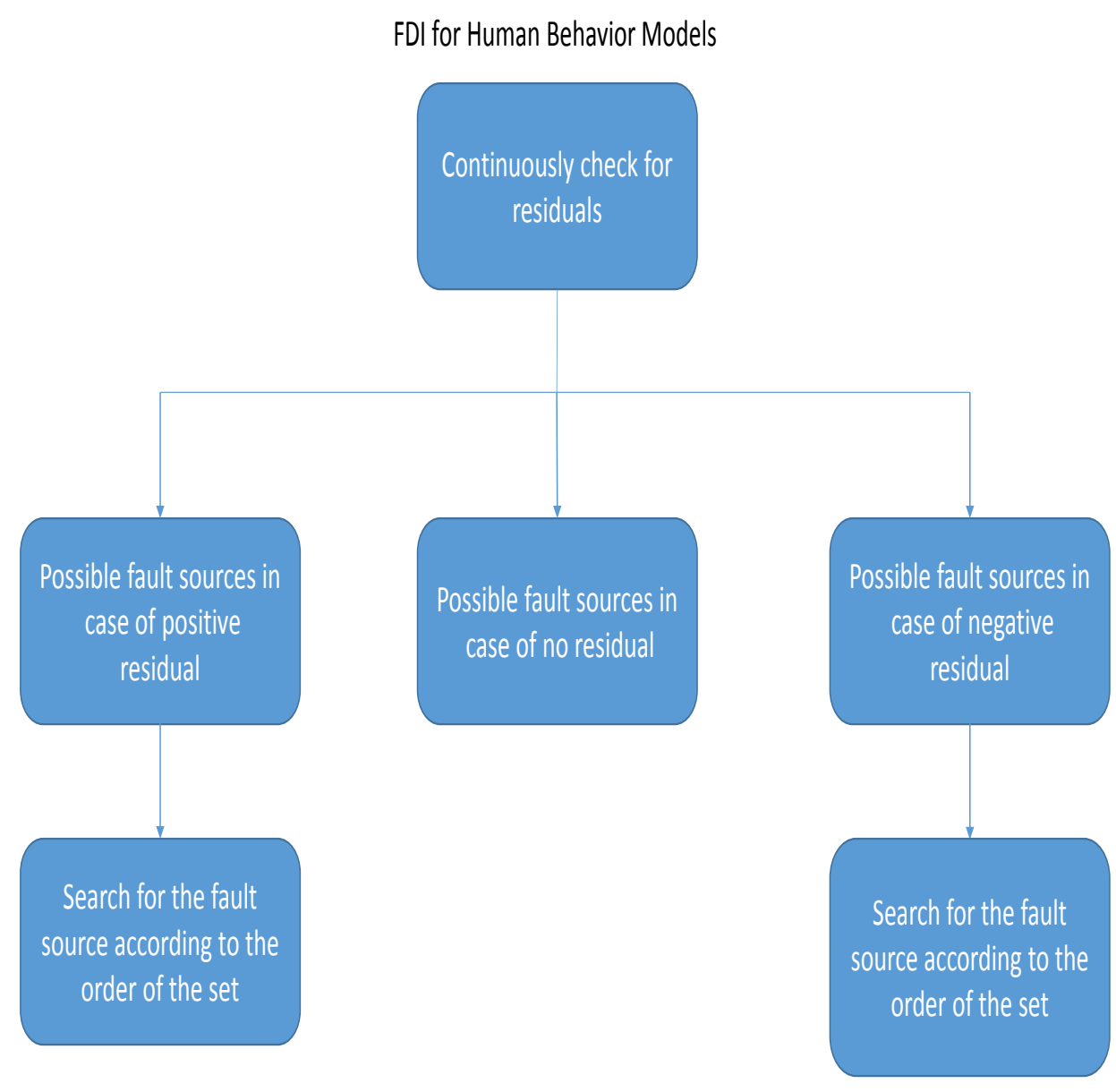

Figure 5.2: FDI scheme for the human behavioral models

In this section, the ARR technique to build a model-based FDI module on human behavior models using Bond Graph is described. Three different human behavior models are considered. First, an FDI for basic Client-Therapist model [75] is designed. Further, ARRs for human behavior models that express the behavioral change based on the theory of planned behavior [10], and, the social cognitive theory [11] are also obtained; these models were proposed in earlier work [4]. 


\subsubsection{Fault Detection in the Basic Client-Therapist Model}

According to the previous work [75], the fluid analogy representation for the interaction between a Client and a Therapist is shown in Figure 5.3. The Bond Graph model is illustrated in Figure 5.4. Here, the Client valence is represented by the storage element type $h_{2}$ on the right and the storage element, $h_{1}$, represents the qualifications of the Therapist. The value in each of these storage elements corresponds to the valence, or affect, of the Client $\left(h_{2}\right)$ and the Therapist $\left(h_{1}\right)$, respectively. The valence of the Therapist is a function of his or her training $\left(S_{1}\right)$ and is regulated by the transformer element $N_{1}$. It is assumed that a better trained Therapist, i.e., more flow in $N_{1}$, would have higher valence. The valence of the Client is affected by the environmental conditions $\left(S_{2}\right)$ and regulated by $N_{2}$. Through the therapy, the valence of the Client and the Therapist are changed because of the flows through the resistor element that is labeled Therapy $(R)$. 


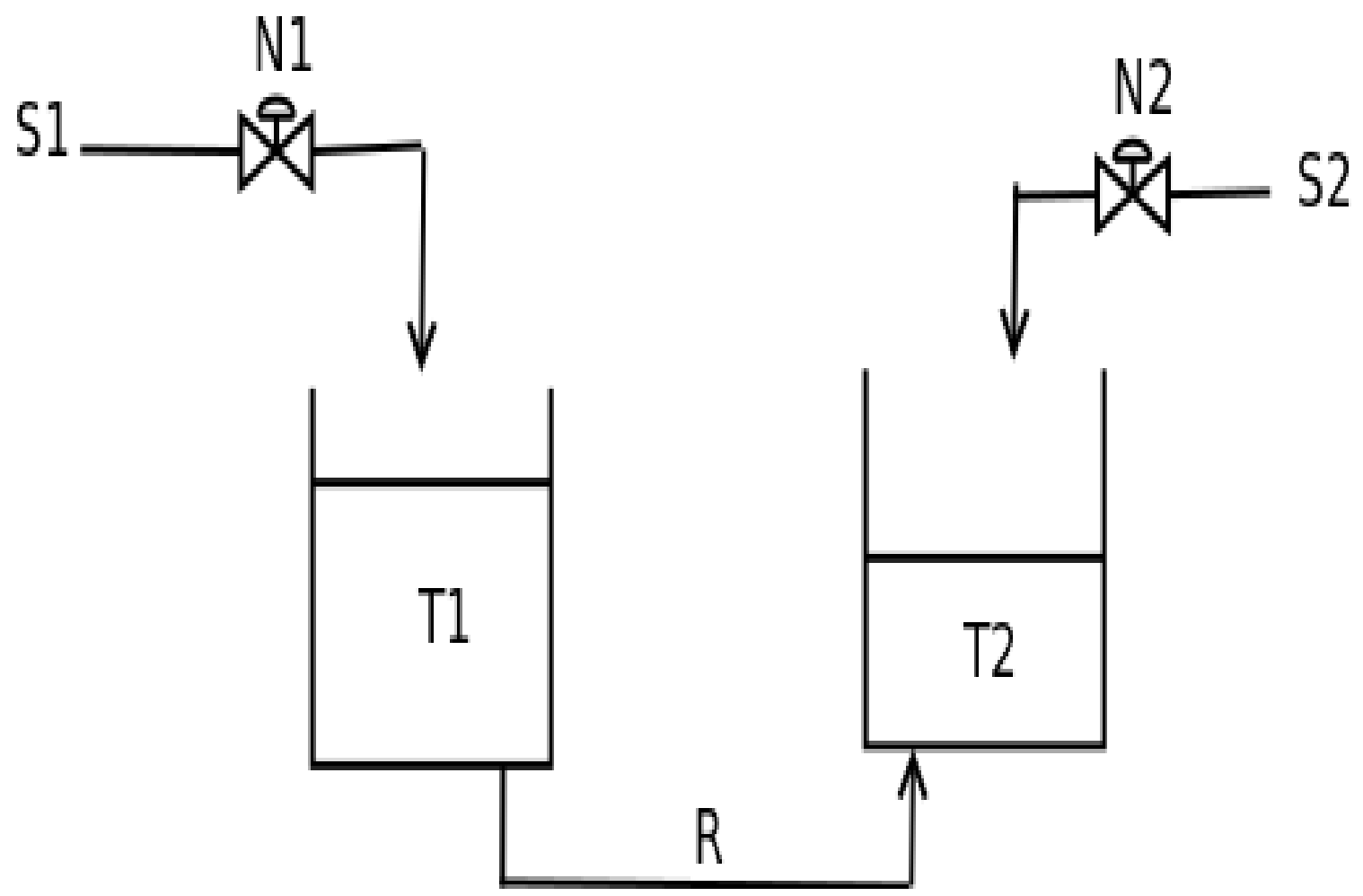

Figure 5.3: Fluid analogy representation for the interaction between a Therapist and a Client. 


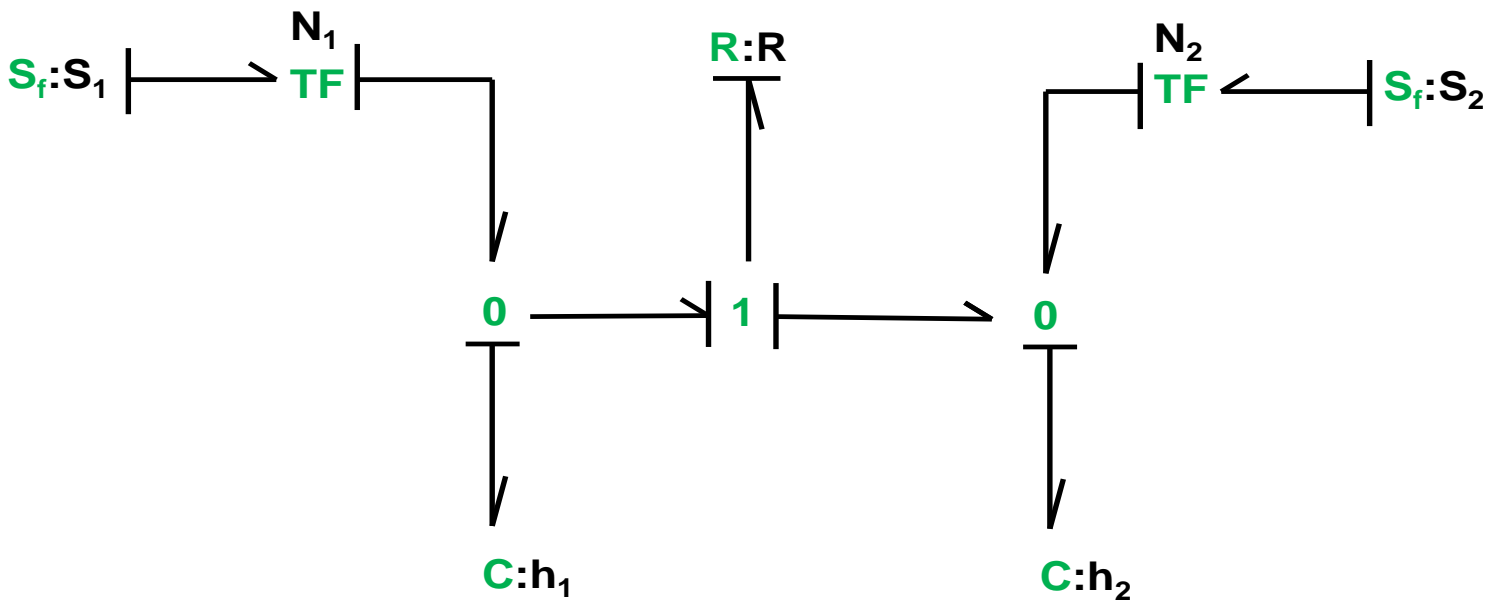

Figure 5.4: Bond graph representation for the Therapist-Client model

The two state variables in the system, i.e., $h_{1}$ and $h_{2}$ can be described by their dynamic equations which specify how these variables change with the time. The equations derived from the Bond Graph model are

$$
\begin{aligned}
& \frac{d h_{1}}{d t}=\frac{S_{1} N_{1}}{C_{1}}-\frac{1}{R C_{1}}\left(h_{1}(t)-h_{2}(t)\right) \\
& \frac{d h_{2}}{d t}=\frac{S_{2} N_{2}}{C_{2}}+\frac{1}{R C_{2}}\left(h_{1}(t)-h_{2}(t)\right)
\end{aligned}
$$

The next step is to identify the observed variables of this system, and express these variables in terms of the parameters of the system. In the basic model of the 
Client-Therapist relation, there is only one observed variable which is the Client's valence, i.e., $h_{2}$. In order to express $h_{2}$ in terms of the system parameters.

$$
h_{2}(t)=\frac{S_{2} N_{2} t}{C_{2}}+\frac{t}{R C_{2}}\left(h_{1}(t)-h_{2}(t)\right)
$$

Now, the next step is to determine the relation between the observed output with all the parameters of the system. First, the parameter $N_{2}$, from Equation (5.2), it is clear that the relation between $h_{2}$ and $N_{2}$ is direct proportional. The Therapy resistor $R$ has inverse proportional relation with $h_{2}$, which can be seen easily from Equation (5.2). Also from Equation (5.2), it can also be seen that $C_{2}$, i.e., the capacitance of the Client's storage is inversely proportional with the $h_{2}$. Finally, the relation between $h_{2}$ and both $C_{1}$ and $N_{1}$, which can be obtained by integrating the first Equation in (5.1), which yields,

$$
h_{1}(t)=\frac{S_{1} N_{1} t}{C_{1}}-\frac{t}{R C_{1}}\left(h_{1}(t)-h_{2}(t)\right)
$$

From which it can be found that $N_{1}$ is directly proportional with $h_{2}$, while $C_{1}$ has inverse proportional relation with $h_{2}$.

From the above relations it can concluded that, $h_{2}$ increases when at least one of $\left(N_{1}, N_{2}\right)$ increases or one of $\left(R, C_{1}, C_{2}\right)$ decreases. And, $h_{2}$ decreases when either $N_{1}$ or $N_{2}$ decreases, or the value of one of the elements $R, C_{1}$ or $C_{2}$, increases.

In the next step of the analysis, all the parameters of the system are must be examined to determine the direction of change in their value, as a result of a fault. 
For instance, the resistor element $R$; based on the physical characteristics, the value of a resistor only increases when faults occur, most of the time. For example, in the fluid analogy model, the resistor of a pipe increases when it has a blockage. We assumed that the resistor of a Client towards therapy only increases when the Client starts to lose trust in the Therapist. On the other hand if the Client's trust towards the therapy increases, the resistor usually stays the same. As for the capacity of the Client's valence, i.e., $C_{2}$, it is assumed that, it can only decrease, based on the analogy of the storage tank, whose capacity can only decrease when some solid elements fall into the tank. On the other hand, based on the physical constraint, the capacity of a tank cannot increase; this assumption is based on the fact that every person has an upper bound for the amount of valence at any given time. This argument is also valid for the Therapist's valence, i.e., $C_{1}$. Regarding the transformers $N_{1}$ and $N_{2}$, it is assumed that the ratios remain constant during the operation of the system, i.e., normally the percentage of the environmental conditions that transfers into the Client's valence is fixed, and, the percentage of the training that transfers into a valence is also fixed for every Therapist.

Based on the above analysis, the set of possible fault sources can be reduced to $\left(C_{1}, C_{2}, R\right)$. Now, an increase in $h_{2}$ can only happen due to a decrease in one of $\left(R, C_{1}, C_{2}\right)$, but it is known that $R$ cannot decrease. Thus, if the value of $h_{2}$ of any Client exceeds the normal expected range of operation, the possible source of this fault is either $C_{1}$ or $C_{2}$. When such fault occurs, the need to order the possible sources of the faults according to their correlation with the observed variable arises. 
In this case, the analyzer suggests an examination of $C_{2}$ first because it has a direct relation with the observed variable $h_{2}$, unlike the element $C_{1}$.

On the other hand, in case the value of $h_{2}$ of any Client goes below the normal expected range of operation, then the possible sources of the fault are again $\left(C_{1}, C_{2}, R\right)$. This can happen due to an increase in one of $\left(R, C_{1}, C_{2}\right)$. Here, $\left(C_{1}, C_{2}\right)$ can be excluded because of the assumption that they cannot increase. This leads one to investigate if there is any blockage in the resistor of the therapy $R$.

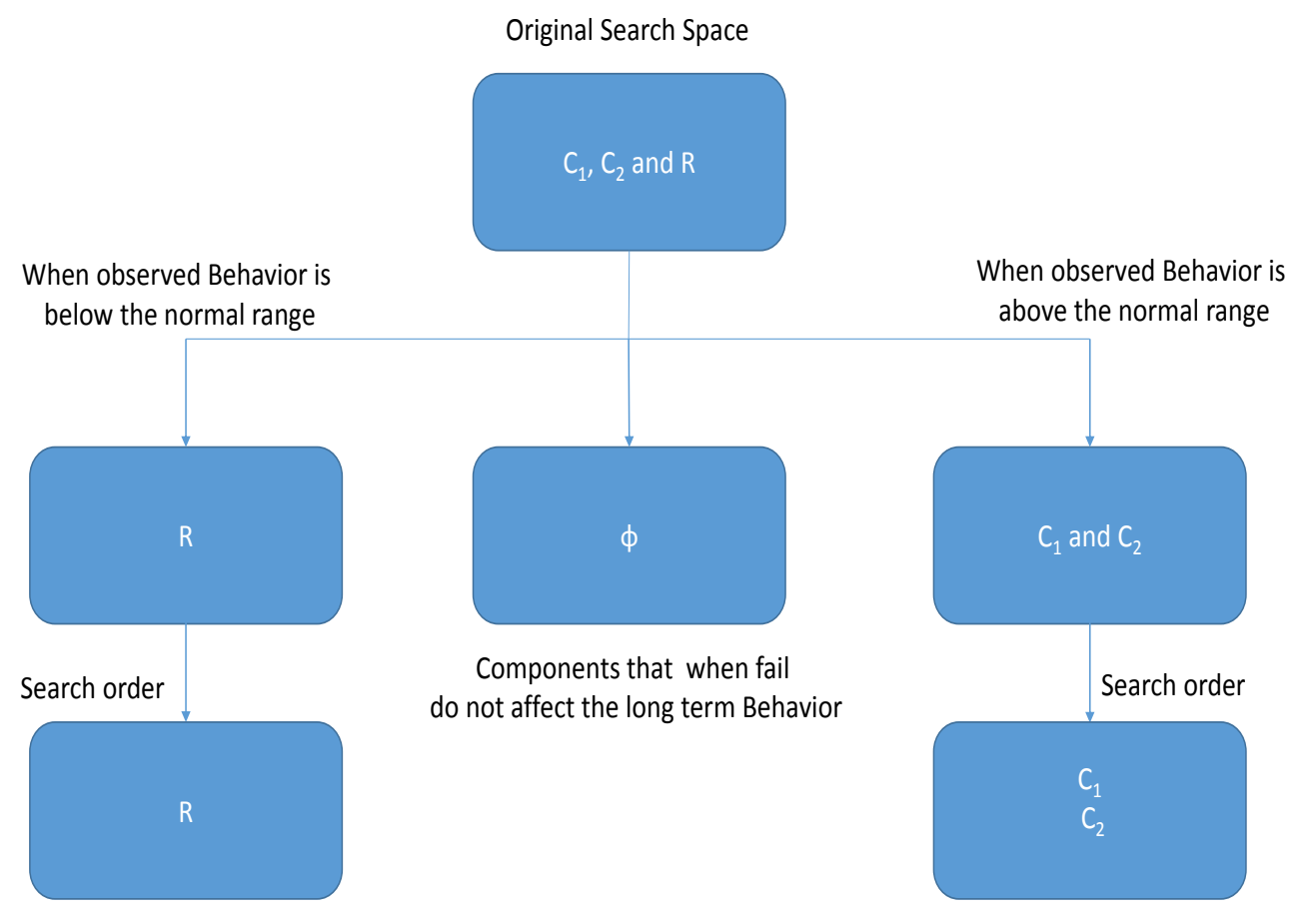

Figure 5.5: FDI scheme for basic Client-Therapist model 


\subsubsection{Fault Detection in the Theory of Planned Behavior Model}

In this section an FDI method in a human behavior model that is constructed based on the theory of planned behavior [10] is presented. The block diagram that describes the theory of planned behavior is shown in Figure 5.6 and the corresponding Bond Graph model is also demonstrated in Figure 5.7.

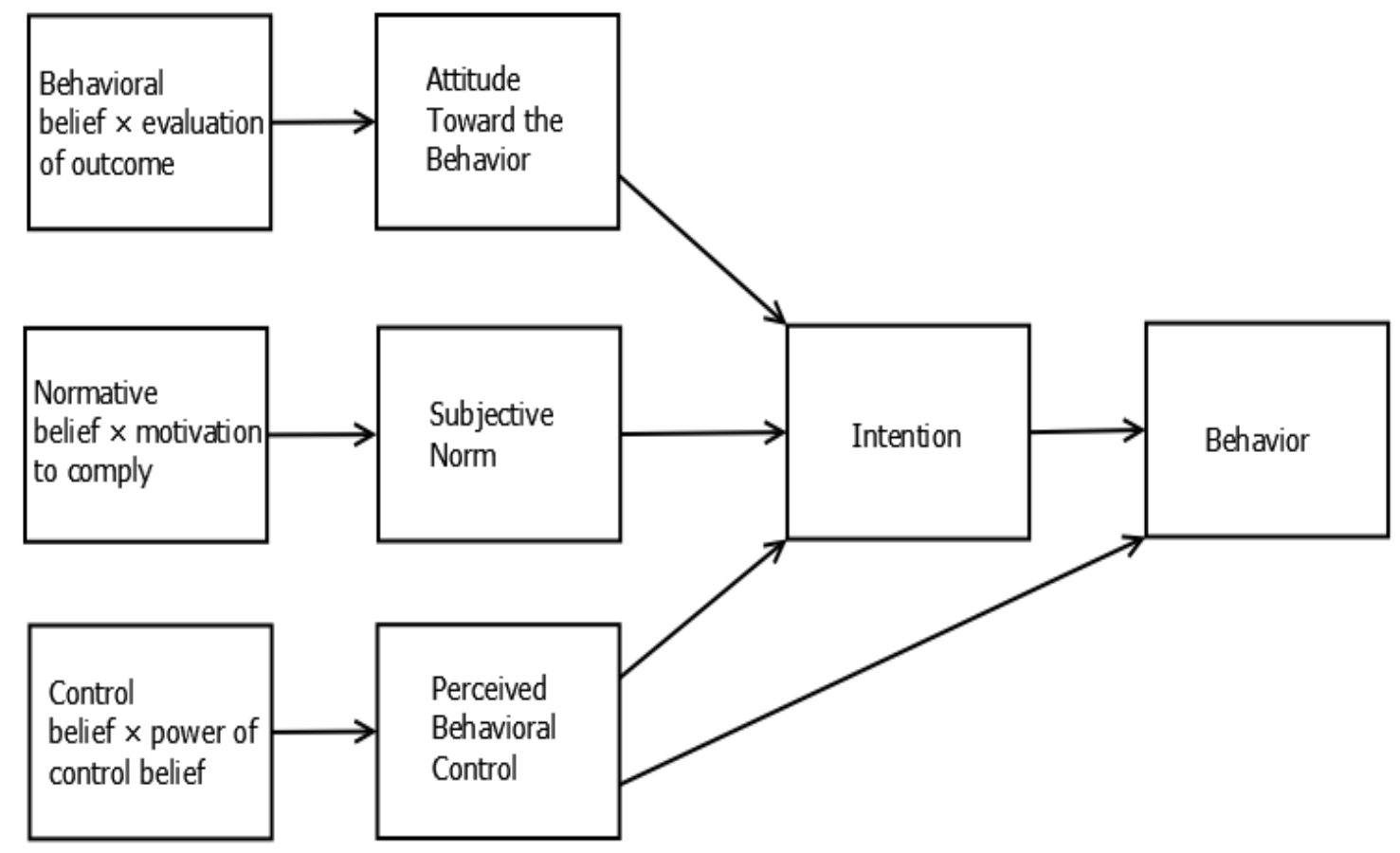

Figure 5.6: Block diagram for the Theory of Planned Behavior.

In order to apply the FDI diagnosis for this model, dynamic equations for the observed variables of the TPB model are determined. The observed output of this model is the Behavior output $\eta_{5}$ and the dynamic behavior can be derived using Bond Graph model [26]. 


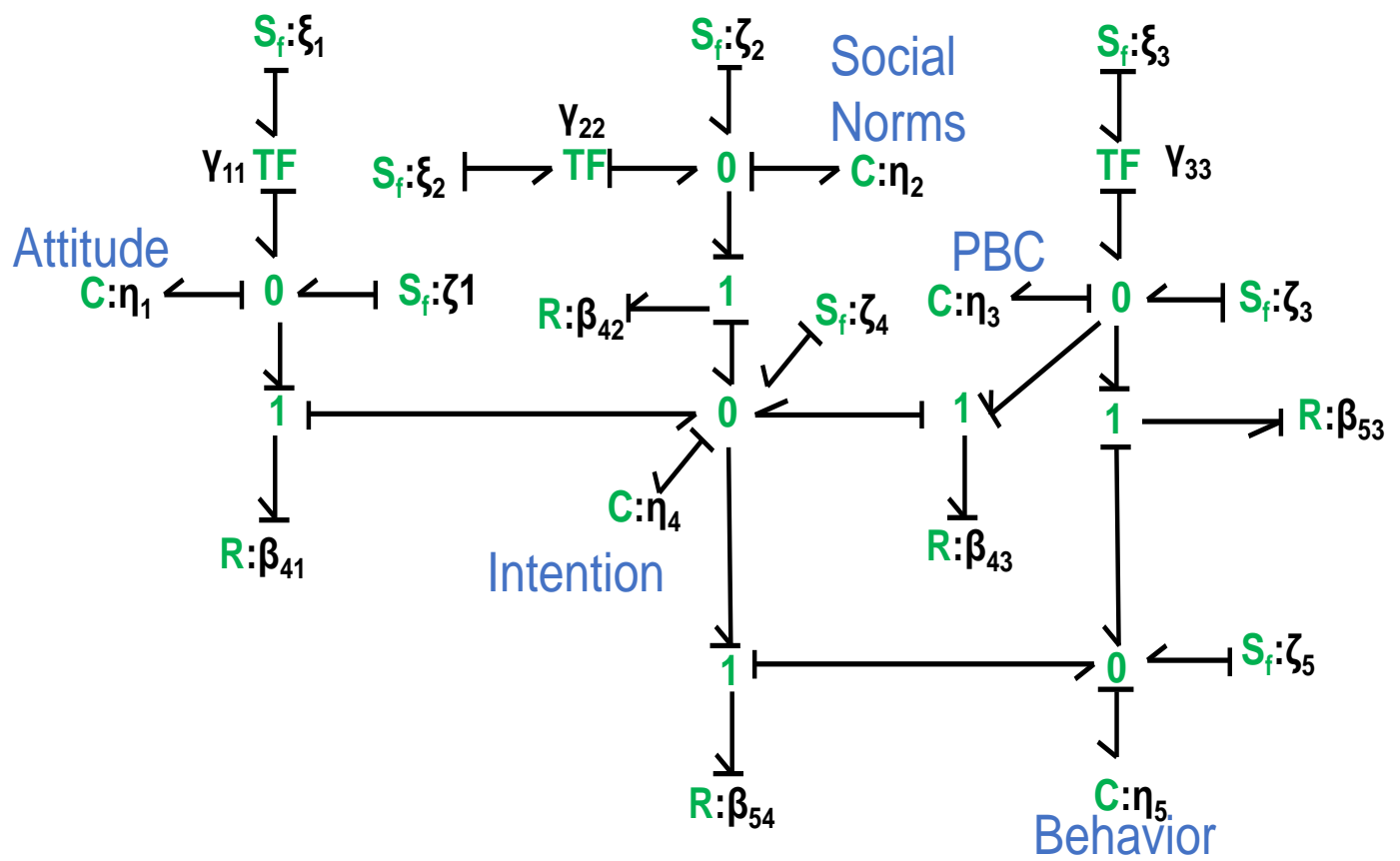

Figure 5.7: Bond graph representation for the Theory of Planned Behavior model

$$
\begin{gathered}
\frac{d \eta_{1}}{d t}=\gamma_{11} \xi_{1}-\frac{1}{\beta_{41}}\left(\eta_{1}-\eta_{4}\right)+\varsigma_{1} \\
\frac{d \eta_{2}}{d t}=\gamma_{22} \xi_{2}-\frac{1}{\beta_{42}}\left(\eta_{2}-\eta_{4}\right)+\varsigma_{2} \\
\frac{d \eta_{3}}{d t}=\gamma_{33} \xi_{3}-\frac{1}{\beta_{43}}\left(\eta_{3}-\eta_{4}\right)-\frac{1}{\beta_{53}}\left(\eta_{3}-\eta_{5}\right)+\varsigma_{3}
\end{gathered}
$$




$$
\frac{d \eta_{4}}{d t}=\frac{1}{\beta_{41}}\left(\eta_{1}-\eta_{4}\right)+\frac{1}{\beta_{42}}\left(\eta_{2}-\eta_{4}\right)+\frac{1}{\beta_{43}}\left(\eta_{3}-\eta_{4}\right)-\frac{1}{\beta_{54}}\left(\eta_{4}-\eta_{5}\right)+\varsigma_{4}
$$

$$
\frac{d \eta_{5}}{d t}=\frac{1}{\beta_{53}}\left(\eta_{3}-\eta_{5}\right)+\frac{1}{\beta_{54}}\left(\eta_{4}-\eta_{5}\right)+\varsigma_{5}
$$

By integrating Equation (5.8) an expression for the observed variable $\eta_{5}$ is presented as follows:

$$
\eta_{5}=\frac{t}{\beta_{53}}\left(\eta_{3}-\eta_{5}\right)+\frac{t}{\beta_{54}}\left(\eta_{4}-\eta_{5}\right)+\varsigma_{5} t
$$

In this model it is assumed that, the sources and transformers from the sources i.e., $\left(\xi_{1}, \xi_{2}, \xi_{3}, \gamma_{11}, \gamma_{22}, \gamma_{33}, \zeta_{1} \ldots \zeta_{5}\right)$ are all external variables which can be identified easily if they had any failure. It is assumed that the resistor elements and the capacities of the storage elements, which control the flow of energy between the storage elements, are internal variables of the system and a failure in these elements will be hard to identify. Hence, our search space is $\left(\beta_{41}, \beta_{42}, \beta_{43}, \beta_{53}, \beta_{54}, C_{1}, C_{2}, C_{3}, C_{4}, C_{5}\right)$.

Following the FDI scheme shown in Figure 5.2, the next step is to find the relations between the observed output $\eta_{5}$ and all these internal parameters. From 
the analysis of Equations 5.4 to 5.9 , it is noted that $\eta_{5}$ has an inverse relation with $\left(\beta_{41}, \beta_{42}, C_{1}, C_{2}, C_{3}, C_{4}, C_{5}\right)$, while the relation is unknown with $\left(\beta_{43}, \beta_{53}, \beta_{54}\right)$.

The next step is to determine the direction in which the value of each parameter changes when failures occur. It is again assumed that, resistor elements can only increase their resistance value due to faults, like blockages, while capacities of storage elements can only decrease in their values due to failures. This step leads to reducing the search span at specific faults.

Now, if $\eta_{5}$ exceeds the normal operational range because of a fault, this can happen due to a decrease in at least one of $\left(\beta_{41}, \beta_{42}, C_{1}, C_{2}, C_{3}, C_{4}, C_{5}\right)$. Since resistor elements do not decrease and the capacities do not increase, we can conclude that, the possible sources of this fault are $\left(\beta_{41}, \beta_{42}\right)$.

On the other hand, if $\eta_{5}$ falls below the normal operational range, this can happen due to an increase in at least one of $\left(\beta_{41}, \beta_{42}, C_{1}, C_{2}, C_{3}, C_{4}, C_{5}\right)$. Since resistor elements do not decrease and the capacities do not increase, the resistor elements are excluded from the search space. Next, the search set is ordered from the highest possibility to the least, the order in this case is $C_{5}, C_{4}, C_{3}, C_{2}$ and $C_{1}$ based on the distance from the output variable. These parameters are checked according to their order to find out the source of the fault.

\subsubsection{Fault Detection in the Social Cognitive Theory Model}

Another theory that has been proposed to explain the human behavioral change is the social cognitive theory [11]. In this section the FDI method is implemented on 


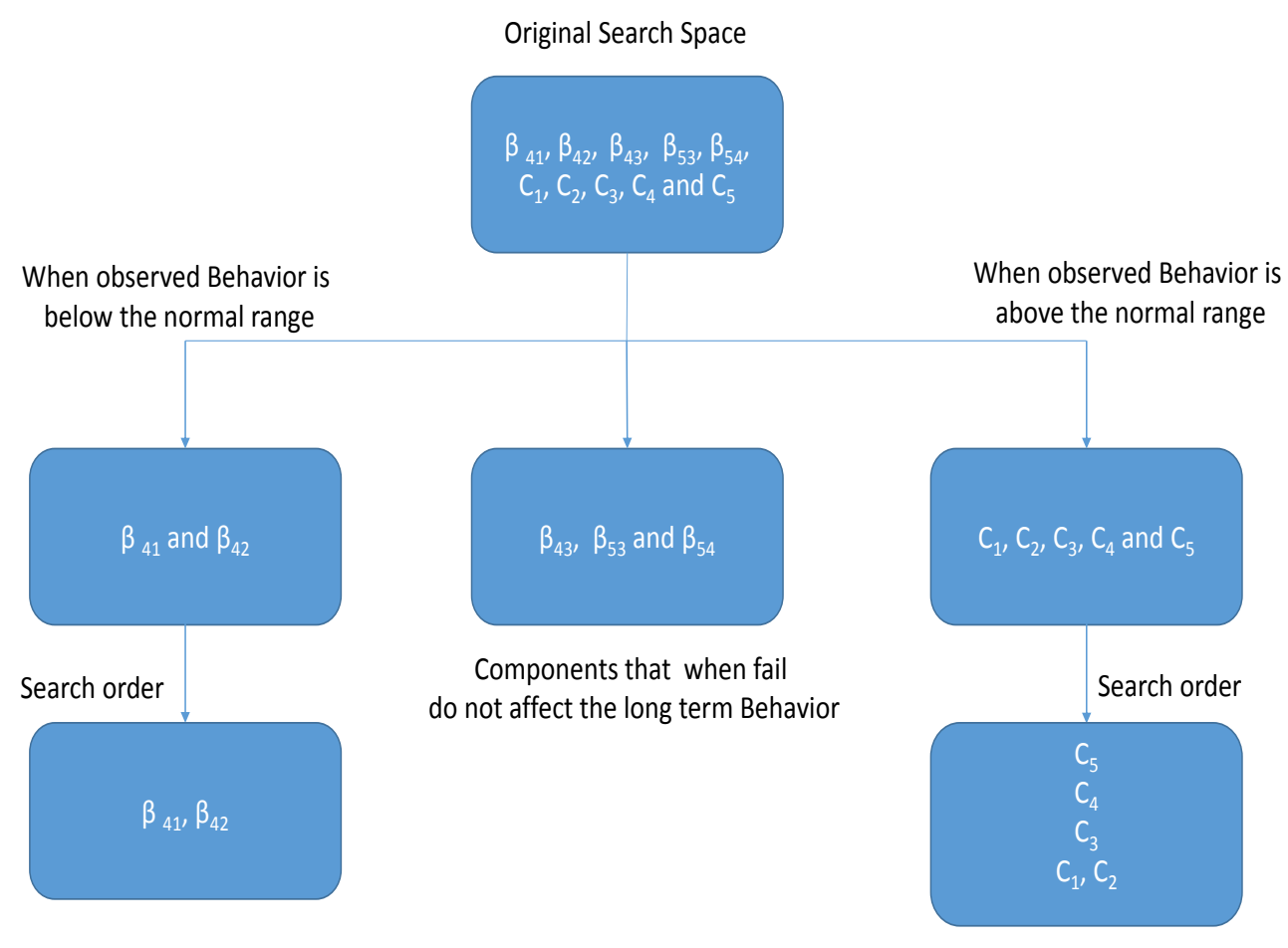

Figure 5.8: FDI scheme for the Theory of Planned Behavior model

the simplified social cognitive theory. The block diagram that describes the simplified social cognitive theory is demonstrated in Figure 5.9 and the corresponding Bond Graph model is constructed in Figure 5.10.

The FDI diagnosis steps are repeated again to obtain the AARs. The observed output is the Behavior output $\eta_{4}$. Again as in the previous section, to find the dynamic equation for the variable $\eta_{4}$, it is necessary to obtain the dynamical equations for all the states of the model; these can be derived using Bond Graph [26]. 


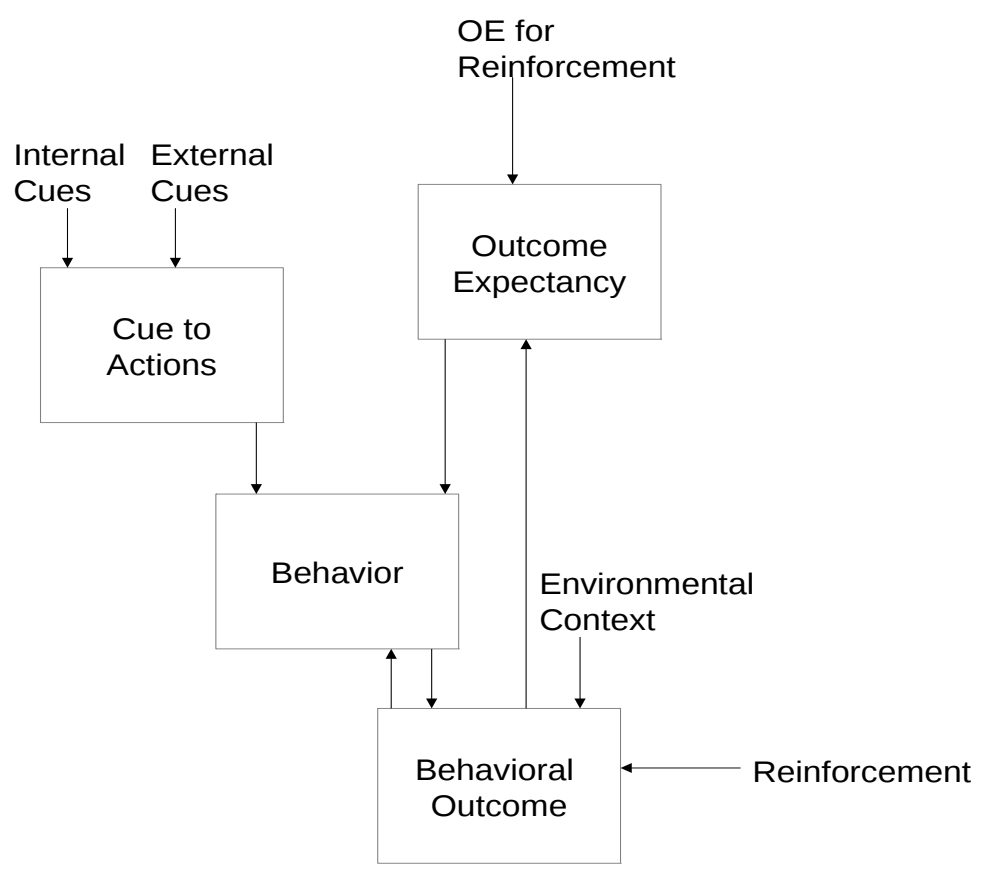

Figure 5.9: Block diagram for the simplified Social Cognitive Theory.

$$
\frac{d \eta_{2}}{d t}=\gamma_{29} \xi_{9}+\frac{1}{\beta_{25}}\left(\eta_{5}-\eta_{2}\right)-\frac{1}{\beta_{42}}\left(\eta_{2}-\eta_{4}\right)+\varsigma_{2}
$$

$$
\frac{d \eta_{4}}{d t}=\frac{1}{\beta_{46}}\left(\eta_{6}-\eta_{4}\right)+\frac{1}{\beta_{42}}\left(\eta_{2}-\eta_{4}\right)+\frac{1}{\beta_{45}}\left(\eta_{5}-\eta_{4}\right)-\frac{1}{\beta_{54}}\left(\eta_{4}-\eta_{5}\right)+\varsigma_{4}
$$




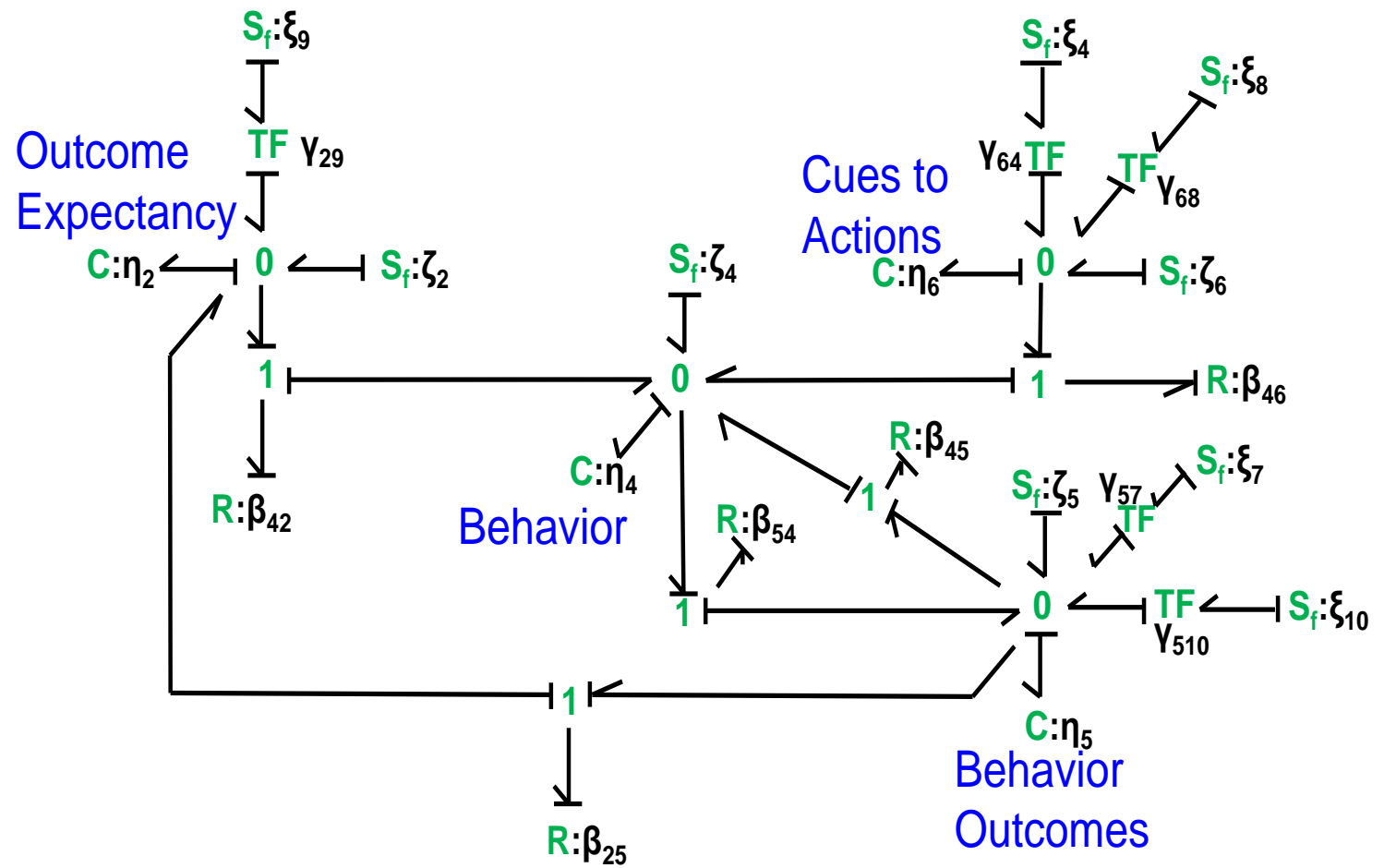

Figure 5.10: Bond graph representation for the simplified Social Cognitive Theory model

$$
\frac{d \eta_{5}}{d t}=\gamma_{57} \xi_{7}+\gamma_{510} \xi_{10}+\frac{1}{\beta_{54}}\left(\eta_{4}-\eta_{5}\right)-\frac{1}{\beta_{45}}\left(\eta_{5}-\eta_{4}\right)-\frac{1}{\beta_{25}}\left(\eta_{5}-\eta_{2}\right)+\varsigma_{5}
$$

$$
\frac{d \eta_{6}}{d t}=\gamma_{64} \xi_{4}+\gamma_{68} \xi_{8}-\frac{1}{\beta_{46}}\left(\eta_{6}-\eta_{4}\right)+\varsigma_{6}
$$

Integrating Equation (5.13) yields an expression for the observed variable $\eta_{4}$ as follows: 


$$
\eta_{4}=\frac{t}{\beta_{42}}\left(\eta_{2}-\eta_{4}\right)+\frac{t}{\beta_{45}}\left(\eta_{5}-\eta_{4}\right)+\frac{t}{\beta_{46}}\left(\eta_{6}-\eta_{4}\right)-\frac{t}{\beta_{54}}\left(\eta_{4}-\eta_{5}\right)+\varsigma_{4} t
$$

The sources and transformers are all external variables which can be identified easily if they had any failure. While the resistor elements and capacitors, which control the flow of energy between the storage elements are internal parameters and they construct our search space for the faults, i.e., $\left(\beta_{25}, \beta_{42}, \beta_{45}, \beta_{46}, \beta_{54}, C_{2}, C_{4}, C_{5}, C_{6}\right)$.

The relations between the observed output $\eta_{4}$ and all these internal parameters can be found to be inverse relation with $\left(\beta_{46}, C_{2}, C_{4}, C_{5}, C_{6}\right)$, and unknown relation with $\left(\beta_{25}, \beta_{42}, \beta_{45}, \beta_{54}\right)$.

The result of the analysis is that when $\eta_{4}$ exceeds the normal operational range, it is because of an increase in one of $\left(\beta_{46}, C_{2}, C_{4}, C_{5}, C_{6}\right)$. The capacities are excluded from the search and, hence, the source of the fault in this case is $\left(\beta_{46}\right)$. In contrast, when $\eta_{4}$ falls below the normal operational range, it happens because of a decrease in at least one of $\left(\beta_{46}, C_{2}, C_{4}, C_{5}, C_{6}\right)$. Here $\left(\beta_{46}\right)$ is excluded and the order of the rest of the possible elements depends on the distance from the output variable. Hence, the order is $\left(C_{4}, C_{5}, C_{2}, C_{6}\right)$. 


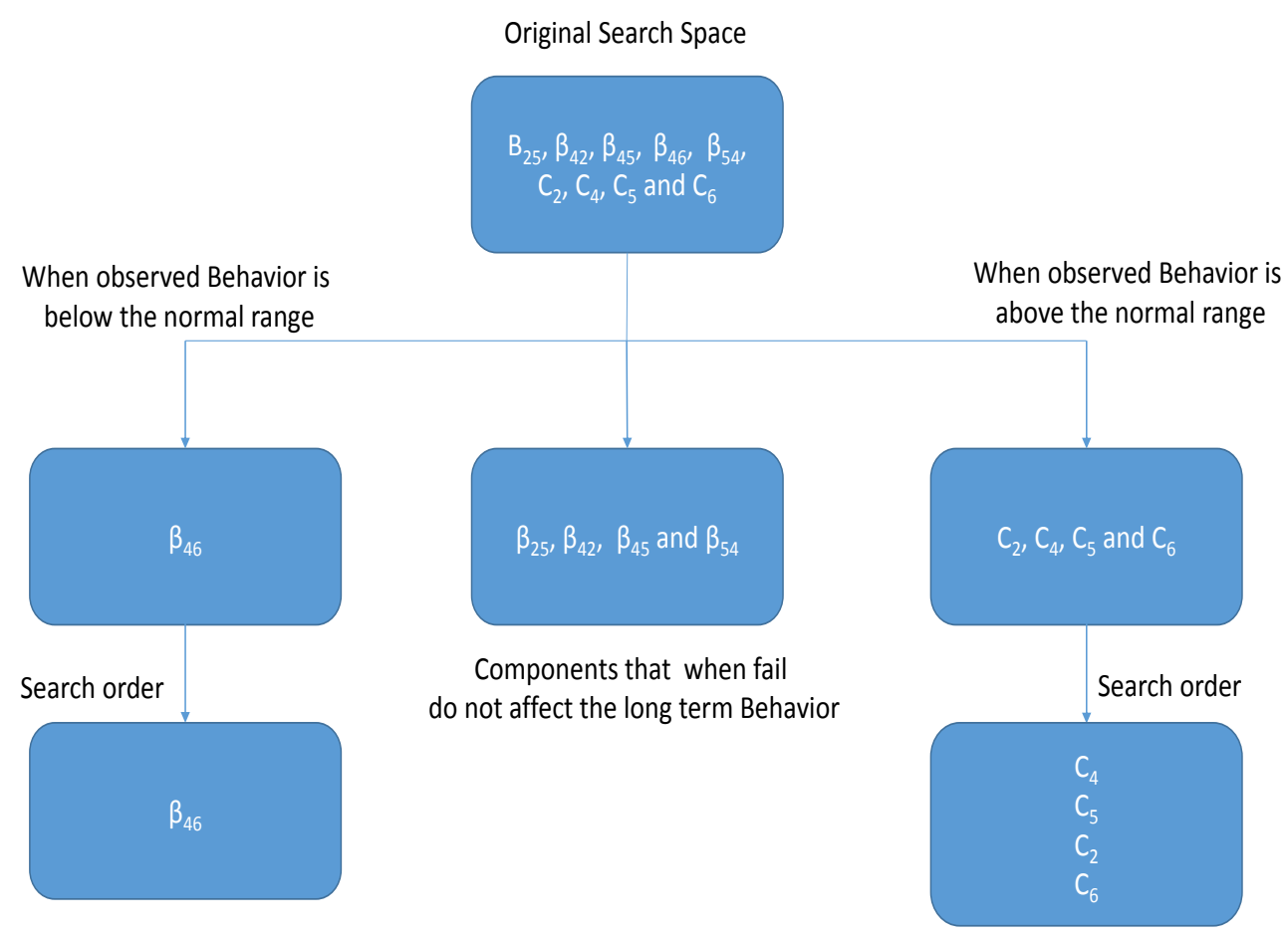

Figure 5.11: FDI scheme for the Social Cognitive Theory model

\subsection{Evaluation}

To evaluate the efficacy of the proposed FDI method for human behavioral models, simulations were used to produce the dynamic behavior of the theory of planned behavior model. Next, one of the parameters was purposely failed in order to simulate an actual fault to examine the FDI method.

A hypothetical 25 year old male participant, Tom, who weighs $126 \mathrm{Kg}$ (277 lbs) is considered. Suppose that Tom is seeking interventions to help with weight loss. 
The well-established weight dynamics model proposed by Hall is used as a reference [65]. This model accepts as input the physical activity level and nutrition level of the participant in intervals of one day; the output of the model is a predicted trajectory for the weight of the participant over a period of up to 180 days. To use this model, a nutrition and physical activity level profiles for Tom are created.

Through therapy, Tom started to increase his physical activity level and maintain healthy nutrition diet, the Therapist suggested that Tom consumes 2200 calories per day and expends about 400 calories per day in addition to his basal metabolic rate through focused exercise activities. These profiles were sent to the weight dynamics model to compute the weight trajectory for Tom for 180 days.

The TPB model shown in Figure 5.7 was used to track Tom's behavior, The external sources $\xi_{1}, \xi_{2}$ and $\xi_{3}$ were set to be equal to 1 , while the transformer ratios $N_{11}, N_{22}$ and $N_{33}$ equal to 0.5 . It is also assumed the capacities of all the storage variables are equal to 20 while the resistor elements are all assumed to be $50 \%$. The initial value for the behavior level $\eta_{5}$ is assumed to be 3 units and the normal operational range for Tom's behavior level is assumed to be \pm 0.5 units of the expected value.

Tom's behavior $\eta_{5}$ directly influences the nutrition and physical activity daily levels, which affect his weight dynamics over the period of 180 days.

Now two cases are considered. First, the $\beta_{41}$ element was forced to fail by increasing the resistance value of $30 \%$ of the original value on the $90^{t h}$ day. The change in Tom's behavior level is shown in Figure 5.12; the effect of this failure in 
his weight is shown in Figure 5.14. In the second case, the capacity of the behavior storage element $C_{5}$ was purposely changed by reducing its value to $70 \%$ of its original value on the $90^{\text {th }}$ day and the corresponding change on Tom's behavior is shown in Figure 5.13 and the change in Tom's weight dynamics is shown in Figure 5.15.

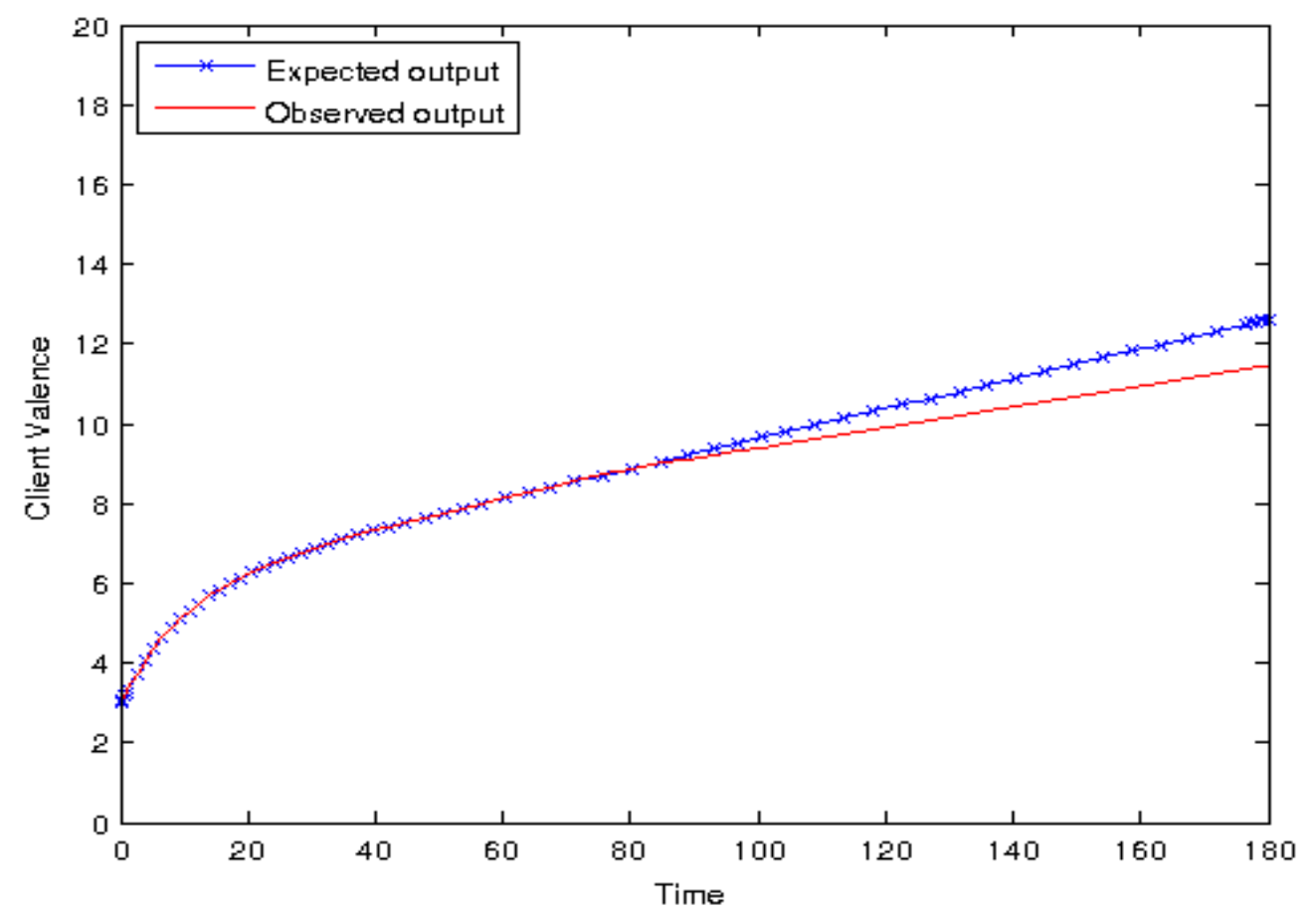

Figure 5.12: Expected and observed Client's valence when a fault happens in the therapy resistor element.

In both cases, when the actual observed weight goes beyond the normal weight change range, the comparator detects the fault and then the analyzer runs to identify the source of the fault. Based on the diagnosis presented in Section 5.1.2, in the first case, the analyzer immediately suggests that the source of the error is one of the resistor elements $\left(\beta_{41}, \beta_{42}\right)$ which means that one of Tom's resistances 


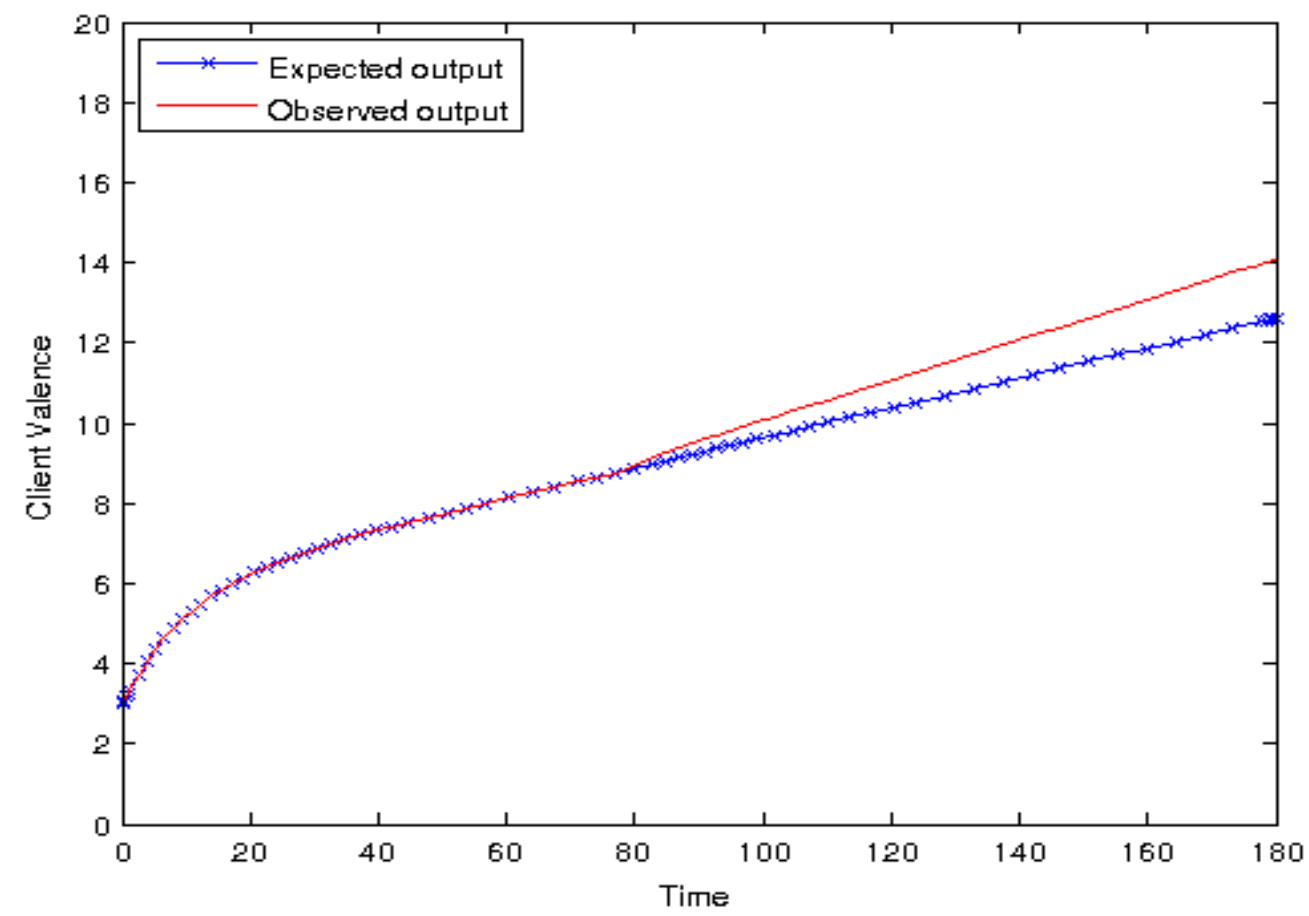

Figure 5.13: Expected and observed Client's valence when a fault happens in the Client's storage capacity element.

that influence his intention based on the attitude or based on his social norms has increased, by checking both of them we find out that $\beta_{41}$ has failed. Hence his behavior is changing and consequently his weight loss started to decrease beyond the safe range.

In case two, the analyzer suggests that, the source is one of the capacity elements, and based on the degree of the relation, it recommends to investigate the element $C_{5}$ first, and it turns out to be the source of the fault. Tom's capacity to store the behavior has decreased due to a failure, hence his behavior level exceeds the normal range, and consequently he is losing weight rapidly, faster than the safe 


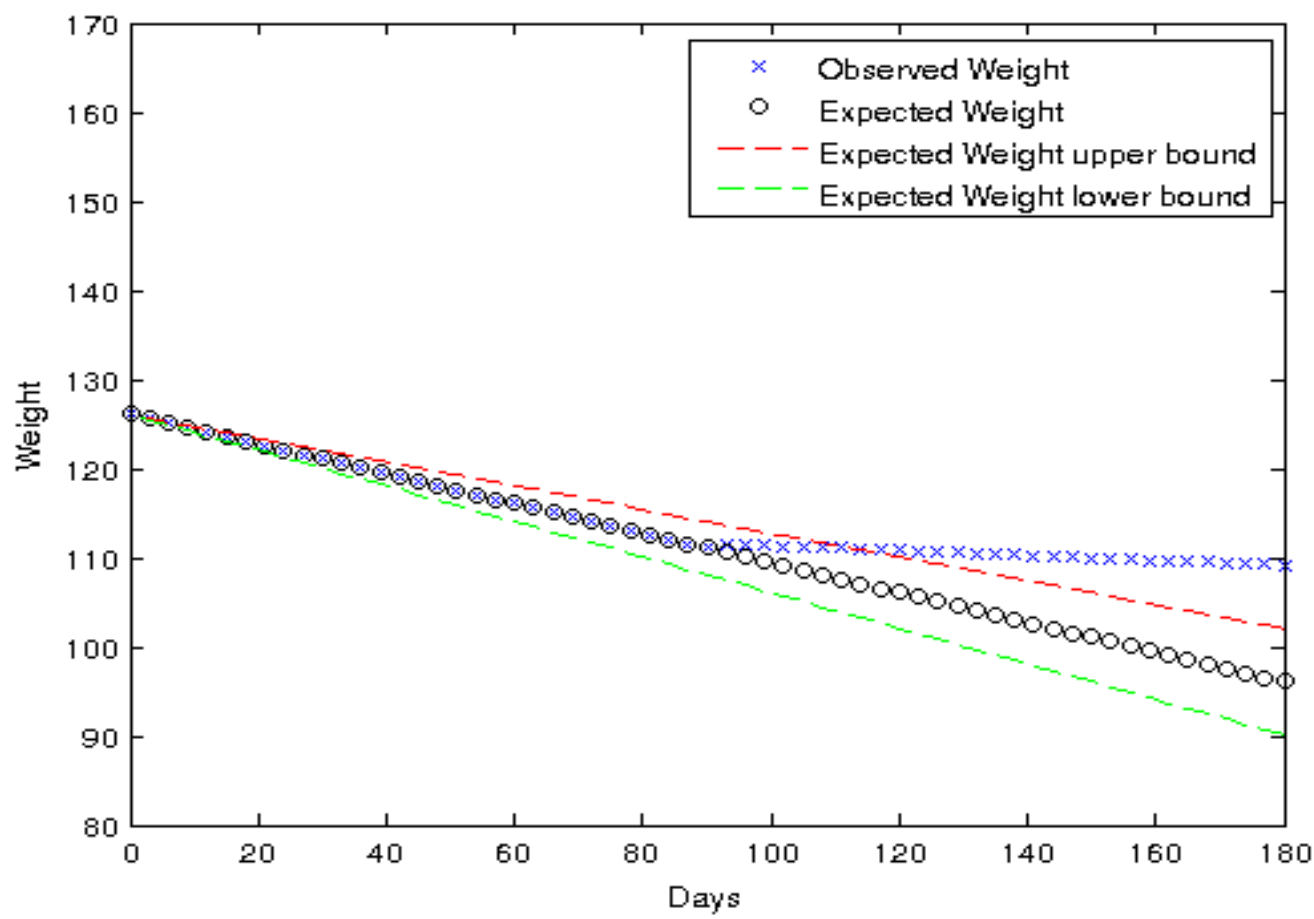

Figure 5.14: Expected and observed Client's weight dynamics when a fault happens in the therapy resistor element.

recommendation.

The results in this Chapter allow us to conclude that, the Bond Graph models presented in this dissertation are also useful for detecting the source of deviations from recommended actions. The ARRs approach was effective for detecting and isolating specific constructs of the theories of human behavior. 


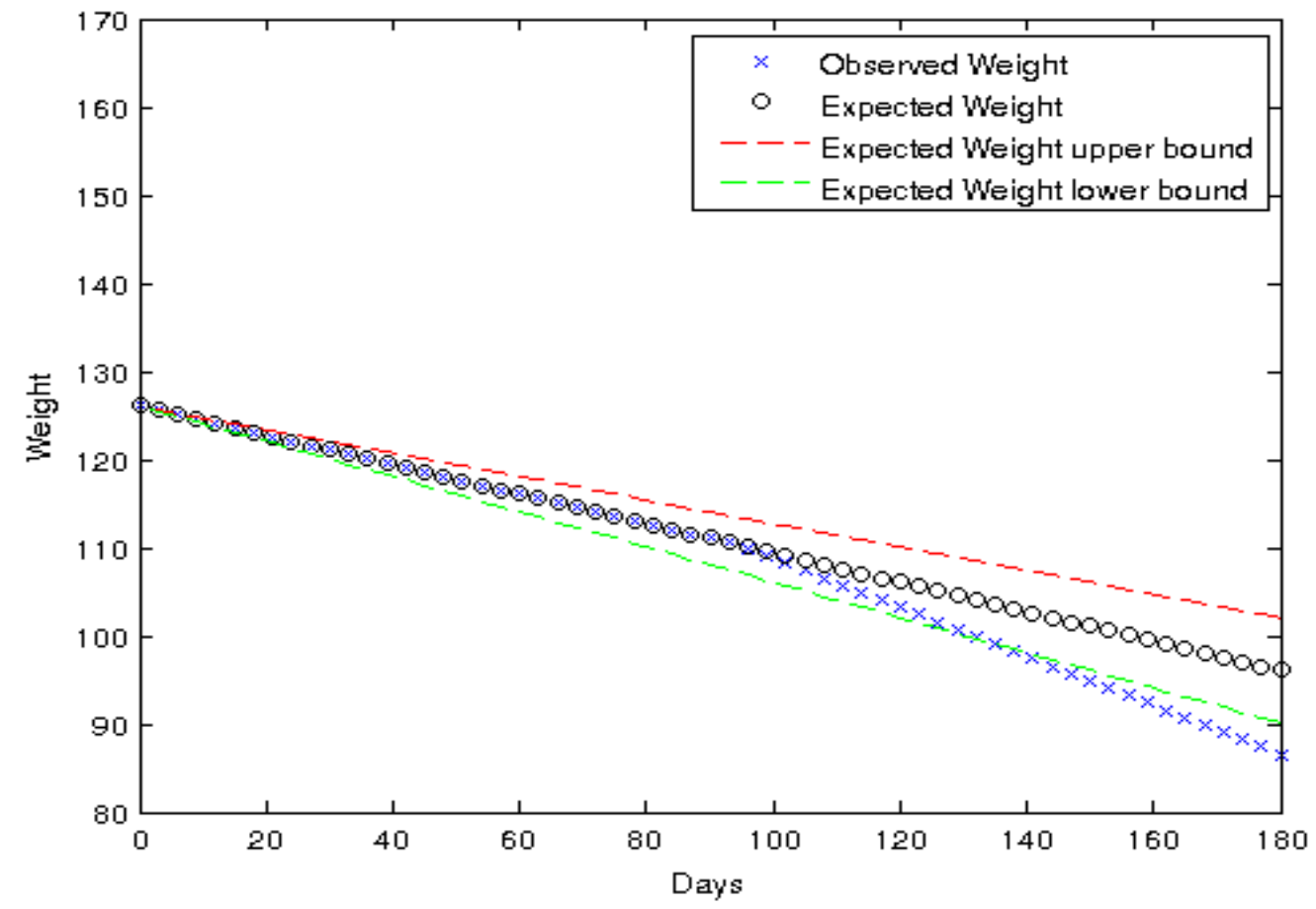

Figure 5.15: Expected and observed Client's weight dynamics when a fault happens in the Client's storage capacity element. 


\section{CHAPTER VI CONCLUSIONS AND FUTURE WORK}

This dissertation has successfully introduced first known Bond Graph models for representing and reasoning about human behavior. These models are actionable and can be incorporated into systems that interact with humans. A comparison of the Bond Graph models and fluid analogy models for human behavior was presented. It was shown that the Bond Graph models do not admit the ambiguities that can arise in the fluid models.

Bond graph models for describing the Client-Therapist interaction were proposed in Chapter 4. These models provided a framework for the Client to choose a suitable Therapist. The method also offered the Therapist new "tools" to regulate the interaction. Client-Therapist interaction models were constructed based on well-known psychology theories, i.e., the theory of planned behavior and the social cognitive theory. The constructs of these theories of human behavior provided an understanding of the observed behaviors at a higher resolution. Furthermore, stability analysis for the Client-Therapist models was presented which provided viable intervention options for different individuals. 
A robust diagnosis scheme was also introduced in Chapter 5 to insure that the proposed models and control schemes support the wellness intervention. The diagnosis method consists of a quantitative model-based Fault Detection and Isolation (FDI) scheme based on the Analytical Redundancy Relations (ARRs). The approach was evaluated using the different Bond Graph models described in this dissertation and it was shown that if the Client's behavior does not match the expected behavior, the FDI scheme effectively suggests the source that caused this deviation from the expected behavior. Such a scheme offers new tools to a therapist and enables them to offer better interventions.

The Bond Graph models for human behavior presented in this dissertation are promising. They form a basis for designing an interactive controllers to empower the personalized wellness management. However there are few ideas that can be pursued in order to extend this work.

The presented models were based on psychology theories. However, there is no methodology to accurately measure the constructs of these theories such as Attitude and Intention. If such method is available to accurately measure these constructs while taking into account the uncertainties, we can reduce the estimations, assumptions and approximations in the proposed models.

Since human beings are different from one another, it is difficult to base human behavior models on only one psychology theory. This is reflected by the different constructs used in the psychology theories. In the future, it is necessary to design methods to find a model that is best suited for a specific individual. 


\section{BIBLIOGRAPHY}

[1] J. E. Navarro-Barrientos, D. E. Rivera, and L. M. Collins, "A dynamical model for describing behavioural interventions for weight loss and body composition change," Mathematical and Computer Modelling of Dynamical Systems, vol. 17, pp. 183-203, 2011.

[2] J. E. Navarro-Barrientos, D. E. Rivera, and L. M. Collins, "A dynamical systems model for understanding behavioral interventions for weight loss.," in $S B P, 2010$.

[3] C. A. Martin, D. E. Rivera, W. T. Riley, E. B. Hekler, M. P. Buman, M. A. Adams, and A. C. King, "A dynamical systems model of social cognitive theory," in American Control Conference, 2014.

[4] A. Mahamadi, S. Sastry, and G. Biswas, "A bond graph approach to modeling human behavior for wellness management," Systems transaction, 2016.

[5] A. Martin, M. Hartman, J. Benson, and C. A., "National health spending in 2014: Faster growth driven by coverage expansion and prescription drug spending," Health Affairs, vol. 35, pp. 150-160, 2016.

[6] D. Umberson and M. J., "Social relationships and health: A flashpoint for health policy," Health and Social Behavior, vol. 51, pp. 54-66, 2010.

[7] C. Martin, S. Deshpande, E. Hekler, and D. E. Rivera, "A system identification approach for improving behavioral interventions based on social cognitive theory," in American Control Conference, 2015.

[8] Y. Dong, D. Rivera, D. M. Thomas, J. E. Navarro-Barrientos, D. S. Downs, J. S. Savage, and L. M. Collins, "A dynamical systems model for improving gestational weight gain behavioral interventions," in American Control Conference, 2012.

[9] D. Lai, K. Cahill, Y. Qin, and J. L. Tang, "Motivational interviewing for smoking cessation," Cochrane Database of Systematic Reviews, vol. 1, p. CD006936, 2010. 
[10] I. Ajzen, "The theory of planned behavior," Organizational Behavior and Human Decision Processes., vol. 50, pp. 179-211, 1991.

[11] A. Bandura, Social foundations of thought and action: A social cognitive theory. Prentice-Hall series in social learning theory., 1986.

[12] R. M. Ryan and E. L. Deci, "Self-determination theory and the facilitation of intrinsic motivation, social development, and well-being," American Psychologist, vol. 55, pp. 68-78, 2000.

[13] J. O. Prochaska, "Decision making in the transtheoretical model of behavior change," Medical Decision Making: an International Journal of the Society for Medical Decision Making, vol. 28, pp. 845-849, 2008.

[14] N. Janz and M. Becker, "The health belief model: A decade later," Health Education Behavior, vol. 11, pp. 1-47, 1984.

[15] N. N. Nandola and D. E. Rivera, "Model-on-demand predictive control for nonlinear hybrid systems with application to adaptive behavioral interventions," in Proceedings of the 49th IEEE Conference on Decision and Control, 2010.

[16] L. Liebovitch, P. Peluso, M. Norman, J. Su, and G. J.M., "Mathematical model of the dynamics of psychotherapy.," Cognitive Neurodynamics., vol. 3, pp. 265$275,2011$.

[17] W. Huitt, "A systems approach to the study of human behavior," in Educational Psychology Interactive. Valdosta, GA, 2012.

[18] D. A. Cohen, R. A. Scribner, and T. A. Farley, "A structural model of health behavior: a pragmatic approach to explain and influence health behaviors at the population level," Preventive medicine, vol. 30, pp. 146-154, 2000.

[19] B. Ziebart, A. L. Maas, J. A. Bagnell, and A. K. Dey, "Human behavior modeling with maximum entropy inverse optimal control," in Human Behavior Modeling, Papers from the 2009 AAAI Spring Symposium, Technical Report SS-09-04, Stanford, California, USA, March 23-25, 2009, 2009.

[20] A. Pentland and A. Lin, "Modeling and prediction of human behavior," Neural Computation, vol. 11, pp. 229-242, 1995.

[21] P. A. Allen, M. Kaufman, A. F. Smith, and R. E. Propper, "A molar entropy model of age differences in spatial memory.," Psychology and Aging, vol. 13, pp. 501-518, 1998. 
[22] M. K. Chippa, S. M. Whalen, F. L. Douglas, and S. Sastry, "Goal-seeking formulation for empowering personalized wellness management," in Medical Cyber Physical Systems Workshop, 2014.

[23] M. Yin and Y. Sun, "Human behavior models for virtual agents in repeated decision making under uncertainty," in Proceedings of the 14th International Conference on Autonomous Agent \& Multiagent Systems (AAMAS'15), 2015.

[24] B. G. Silverman, G. Bharathy, K. O’Brien, and J. Cornwell, "Human behavior models for agents in simulators and games: Part ii: Gamebot engineering with pmfserv," Presence: Teleoper. Virtual Environ, vol. 15, pp. 163-185, 2006.

[25] B. G. Silverman, M. Johns, J. Cornwell, and K. O'Brien, "Human behavior models for agents in simulators and games: Part i: Enabling science with pmfserv," Presence: Teleoper. Virtual Environ., vol. 15, pp. 139-162, 2006.

[26] J. F. Broenink, "Introduction to physical systems modeling with bond graphs," in in the SiE whitebook on Simulation Methodologies, 1999.

[27] H. M. Paynter, Analysis and design of engineering systems. M.I.T. Press, Cambridge, 1961.

[28] P. J. Gawthrop and G. P. Bevan, "Bond-graph modeling a tutorial introduction for control engineers," IEEE Control Systems Magazine, vol. 27, pp. 24-45, 2007.

[29] P. C. Breedveld, "Modeling and simulation of dynamic systems using bond graphs," in Control Systems, Robotics and Automation - Modeling and System Identification I, EOLSS Publishers Co. Ltd./UNESCO, 2008.

[30] W. Broutzky, "Bond graph modelling and simulation of multidisciplinary systems - an introduction," Simulation Modelling Practice and Theory, vol. 17, pp. 3-21, 2009.

[31] P. Gawthrop, "Bond graphs: A representation for mechatronic systems," Mechatronics, vol. 1, pp. 127-156, 1991.

[32] G. N. Twilhaar, "Network representation of electromagnetic fields and forces using generalized bond graphs," Journal of the Franklin Institute, vol. 319, pp. 183-200, 1985.

[33] D. Karnopp, "Bond graph models for electromagnetic actuators," Journal of the Franklin Institute, vol. 319, pp. 173-181, 1985. 
[34] D. Karnopp, "Understanding magnetic and electrostatic actuators using bond graphs and a mechanical model," Journal of the Franklin Institute, vol. 317, pp. 227-234, 1984.

[35] H. Engja, "Modeling and simulation of heat exchanger dynamics using bond graphs.," in 2nd European Simulation Congress, 1986.

[36] S. Azarbaijani and D. Karnopp, "Bond graph structural models for heat exchangers," American Society of Mechanical Engineers, vol. 2, pp. 138-141, 1980.

[37] J. W. Brewer and M. Hubbard, "Bond-graphs of solar-regulated buildings part3. comparison of modal and finite-difference simulations," in Simulations Councils, 1978.

[38] G. A. Artcca and P. G. Mczcy, "Molecular conformations and molecular shape: A discrete characterization of continua of van der waals surfaces," International Journal of Quantum Chemistry, vol. 34, pp. 517-526, 1988.

[39] J. L. Wyatt, "Network representation of reaction-diffusion systems far from equilibrium," Computer Programs in Biomedicine, vol. 8, pp. 180-195, 1978.

[40] J. J. Beaman, "A dynamic model of a pressure swing oxygen generation system," Journal of Dynamic Systems, Measurement, and Control, vol. 107, pp. 111-116, 1985.

[41] D. Karnopp, "Bond graph models for electrochemical energy storage : electrical, chemical and thermal effects.," Journal of the Franklin Institute, vol. 327, pp. 983-992, 1990.

[42] P. G. Van de Veen, W. Van der Tempel, and J. De Vreiss, "Bond graph modeling and simulation of the dynamic behavior of above-knee prosthesis," Prosthetics and Orthotics International, vol. 11, pp. 65-70, 1987.

[43] J. P. Montoya, K. R. Diller, J. J. Beaman, and P. C. Breedfield, "Network thermodynamic model for coupled membrane transport: Application to cell cryopreservation," in Thermal Engineering Joint Conference, 1987.

[44] D. Hrovat and W. Tobler, "Bond graph modeling of automotive power trains," Journal of the Franklin Institute, vol. 328, pp. 623-662, 1991.

[45] C. Bidard, "Kinematic structure of mechanisms: a bond graph approach.," Journal of the Franklin Institute, vol. 328, pp. 901-915, 1991. 
[46] W. Drozdz and H. Pacejka, "Development and validation of a bond graph handling model of an automobile.," Journal of the Franklin Institute, vol. 328, pp. 941-957, 1991.

[47] I. J. BuschVishniac and H. M. Paynter, "Bond graph models of sound and vibration systems," The Journal of the Acoustical Society of America, vol. 85, pp. 1750-1758, 1989.

[48] P. L. Graf and R. Shoureshi, "Modeling and implementation of semi-active hydraulic engine mounts," Journal of Dynamic Systems, Measurement, and Control, vol. 110 , pp. 422-429, 1988.

[49] A. M. Karmel, "Design and analysis of a transmission hydraulic system for an engine-flywheel hybrid-vehicle.," in American Control Conference, 1988.

[50] J. W. Brewer, "Progress in the bond graph representation of economics and population dynamics," Franklin Institute, vol. 328, pp. 675-696, 1991.

[51] J. W. Brewer, P. P. Craig, M. Hubbard, and K. E. Watt, "The bond-graph method for technological forecasting and resource policy analysis," Energy, vol. 7, pp. 505-537, 1982.

[52] J. W. Brewer, "Bilinear, dynamic single-ports and bond graphs of economic systems," Franklin Institute, vol. 313, pp. 185-196, 1982.

[53] R. C. Rosenberg, W. Feurzeig, and P. Wexelblat, "Bond graphs and enport in elementary physics instruction," IEEE Transactions on Man-Machine Systems, vol. 11, pp. 170-174, 1970.

[54] I. Potter and M. Sunman, "Thresholdless redundancy management with arrays of skewed instruments," tech. rep., AGARD, 1977.

[55] D. Maquin, V. Cocquempot, J. Cassar, M. Staroswiecki, and J. Ragot, "Generation of analytical redundancy relations for fdi purposes," in IFAC Symposium on Diagnostics for Electrical Machines, Power Electronics and Drives, SDEMPED'9'7, 1997.

[56] A. Shumsky, "Robust analytical redundancy relations for fault diagnosis in nonlinear systems," Asian Journal of Control, vol. 4(2), pp. 159-170, 2002. 
[57] W. Borutzky, "Residual bond graph sinks for numerical evaluation of analytical redundancy relations in model based single fault detection and isolation," in Modelling methodologies and simulation. Key technologies in academia and industry., 2006.

[58] M. Khemliche, A. Badoud, and S. Latreche, "Bond graph modelling for fault detection and isolation of an ultrasonic linear motor," Leonardo Journal of Sciences, vol. 16, pp. 125-144, 2010.

[59] T. Mekki, S. Triki, and A. Kamoun, "Diagnosis of switching systems using hybrid bond graph.," Computing Research Repository, vol. 10(1), p. abs/1312.2047, 2013.

[60] P. Feenstra, E. Manders, P. Mosterman, G. Biswas, and R. Barnett, "Modeling and instrumentation for fault detection and isolation of a cooling system," in IEEE Southeastcon, 2000.

[61] I. Roychoudhury, G. Biswas, and X. Koutsoukos, "Designing distributed diagnosers for complex continuous systems," IEEE Transactions on Automation Science and Engineering, vol. 6(2), pp. 277-290, 2009.

[62] G. Biswas, X. Yu, and K. Debelak, "A formal modeling scheme for continuousvalued systems: Focus on diagnosis," in International Joint Conference on Artificial Intelligence, 1992.

[63] W. Borutzky, "Analytical redundancy relations from bond graphs of hybrid system models," in 5th International Conference on Integrated Modelling and Analysis in Applied Control and Automation, 2011.

[64] G. Calderon-Espinoza, J. Armengol, J. Vehi, and E. Gelso, "Dynamic diagnosis based on interval analytical redundancy relations and signs of the symptoms," AI Communications, vol. 20, pp. 39-47, 2007.

[65] C. Chow and K. Hall, "The dynamics of human body weight change," PLoS Computational Biology, vol. 4, p. e1000045, 2008.

[66] P. Pirolli1, S. Mohan, R. Yang, A. Venkatakrishnan, M. Silva, M. Youngblood, A. Ram, , and L. Nelson, "User modeling and planning for improving self-efficacy and goal adherence in mhealth," in 2nd Behaviour Change Conference: Digital Health and Wellbeing, 2016.

[67] T. A. Shimp and A. Kavas, "The theory of reasoned action applied to coupon usage," Journal of Consumer Research, vol. 11, pp. 795-809, 1984. 
[68] A. Mahamadi and S. Sastry, "A bond graph approach for wellness management based on the client-therapist model.," in The 14th International Conference on Cognitive Modeling, 2016.

[69] P. Mosterman and G. Biswas, "A theory of discountinuities in physical system models," Franklin Institute, vol. 335B, pp. 401-439, 1998.

[70] A. Mahamadi and S. Sastry, "Bond graph models for human behavior," in Conference of Basic Sciences and Engineering Studies (SGCAC), 2016.

[71] W. L. Brogan, Modern control theory. Prentice Hall, Inc., 1991.

[72] F. Fairman, Linear Control Theory: The State Space Approach. Wiley, 1998.

[73] H. Trentelman, A. Stoorvogel, and M. Hautus, Control Theory for Linear Systems. Springer Verlag, 2001.

[74] A. K. Samantaray and B. O. Bouamama, Model-based Process Supervision: A Bond Graph Approach. Springer-Verlag London, 2008.

[75] A. Mahamadi and S. Sastry, "A bond graph approach for modeling the clienttherapist relation.," in The 2nd International Conference on Health Informatics and Medical Systems, 2016. 